\title{
Motion by Curvature of Planar Networks
}

\author{
CARLO MANTEGAZZA - MATTEO NOVAGA - \\ VINCENZO MARIA TORTORELLI
}

\begin{abstract}
We consider the motion by curvature of a network of smooth curves with multiple junctions in the plane, that is, the geometric gradient flow associated to the length functional.

Such a flow represents the evolution of a two-dimensional multiphase system where the energy is simply the sum of the lengths of the interfaces, in particular it is a possible model for the growth of grain boundaries.

Moreover, the motion of these networks of curves is the simplest example of curvature flow for sets which are "essentially" non regular.

As a first step, in this paper we study in detail the case of three curves in the plane meeting at a single triple junction and with the other ends fixed. We show some results about the existence, uniqueness and, in particular, the global regularity of the flow, following the line of analysis carried on in the last years for the evolution by mean curvature of smooth curves and hypersurfaces.
\end{abstract}

Mathematics Subject Classification (2000): 53C44 (primary); 53A04, 35K55 (secondary).

\section{CONTENTS:}

1. Introduction and basic definitions $\ldots \ldots \ldots \ldots \ldots \ldots \ldots \ldots \ldots \ldots \ldots \ldots \ldots$

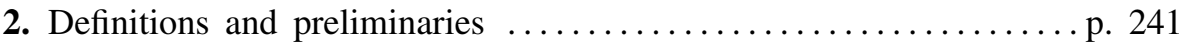

3. Small time existence and a priori estimates ................ p. 249

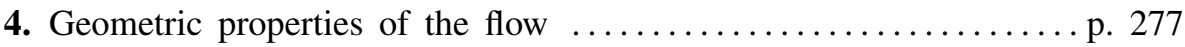

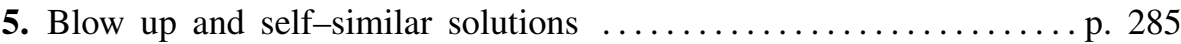

5.1. Classification of homothetic flows of triods ............ 286

5.2. Classification of translating flows of triods ............. p. 287

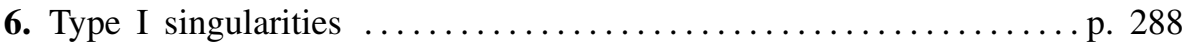

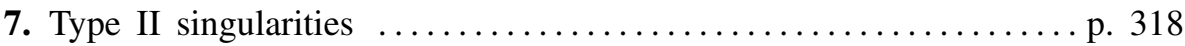

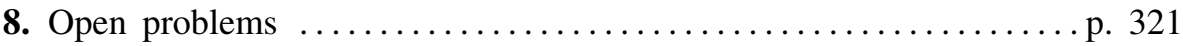

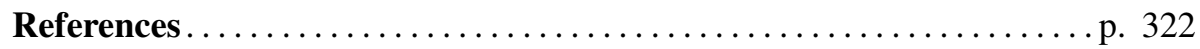

Pervenuto alla Redazione il 9 maggio 2003. 


\section{1. - Introduction and basic definitions}

In this work we address the problem of the motion by curvature of a network of curves in the plane, where by network of curves we mean a connected planar graph without self-intersections.

The evolution by curvature of such a network is the geometric gradient flow with respect to the energy given by the Length functional which is simply the sum of the lengths of all the curves of the network (see [10]).

We point out two motivations to study this evolution. The first is the analysis of models of two-dimensional multiphase systems, where the problem of the structure and regularity of the interfaces between different phases arises naturally. As an example, the model where the energy of a configuration is simply the total length has proven useful in the analysis of the growth of grain boundaries, see [10], [11], [20], [30], the papers by Herring and Mullins in [9] and http://mimp.mems.cmu.edu.

The second motivation is more theoretical: the evolution of such a network of curves in the plane is the simplest example of motion by mean curvature of a set which is essentially singular. In the literature there are various generalized definitions of flow by mean curvature for non regular sets (see [3], [10], [14], [18], [27], [36], for instance). All of them are fairly general, but usually lack uniqueness and a satisfactory regularity theory, even in simple situations.

Inspired by Grayson's Theorem in [19], stating that any smooth closed curve embedded in $\mathbb{R}^{2}$ evolves by curvature without singularities before vanishing, and by the new approach to such result by Huisken in [26], one can reasonably expect that an "embedded" network of smooth curves does not develop singularities during the flow if its "topological structure" does not change (we will be more precise about this point in the sequel) and asymptotically converges to a critical configuration for the Length functional.

Moreover, in [26] it is also shown that the motion by curvature of a single embedded curve in a strip of $\mathbb{R}^{2}$, with its end points fixed to be some $P^{1}$ and $P^{2}$ on the boundary of the strip, evolves smoothly and approaches the segment connecting the two points $P^{1}, P^{2}$. We can see this case as a very special positive example of motion of a network and we will try to follow the line of analysis traced in that paper.

We consider a connected network $\mathbb{S}=\cup_{i=1}^{n} \sigma^{i}$, composed of a finite family of smooth curves $\sigma^{i}(x):[0,1] \rightarrow \bar{\Omega}$, where $\Omega$ is a smooth open convex subset of $\mathbb{R}^{2}$, that can intersect each other or self-intersect only at their end points. We call "multi-points" the vertices $O^{1}, O^{2}, \ldots, O^{n} \in \Omega$ of such smooth graph $\mathbb{S}$, where the order is greater than one. Moreover, we assume that all the other ends of the curves (if present) have to coincide with some points $P^{l}$ on the boundary of $\Omega$.

The problem is then to analyse the existence, uniqueness, regularity and asymptotic behavior of the evolution by curvature of such a network, under the constrain that the end points $P^{l} \in \partial \Omega$ stay fixed. 
Clearly, one can also set an analogous "Neumann" problem, requiring that, instead of being fixed, at the "free" ends the curves intersect orthogonally the boundary of $\Omega$.

REMARK 1.1. If $\Omega$ is a generic smooth open subset of $\mathbb{R}^{2}$, possibly non convex, it could happen that during the evolution one of the curves "hits" the boundary of $\Omega$ with a point different from its end point. Then, for sake of simplicity, we assume the convexity of the domain since this condition excludes a priori such an event (Proposition 4.1).

The hypothesis that the points $P^{l}$ stay on the boundary and not inside $\Omega$, is also in this spirit. If the fixed end points are inside the domain the interior of a curve could possibly "touch" one of them, forming a loop and losing the "embeddedness" of the network.

The convexity assumption and the fact that $P^{l} \in \partial \Omega$ avoid this possibility, as we will see in Section 4.

In such a generality, although simplified in this way, this problem shares various complications related to the multi-points.

As previously underlined, the existing weak definitions of curvature motion do not give uniqueness of the flow, or allow "fattening" phenomena (see [18], for instance) which we would like to avoid, as they seems quite extraneous to our setting. Among the existing notions, the most suitable to our point of view is Brakke's one (see Definition 2.8 and the subsequent discussion), which also lacks uniqueness but maintains the (Hausdorff) dimension of the sets, excluding at least the event of fattening. In what follows this definition is the only that we consider in relation to the evolution of networks, in particular, our flows are Brakke flows.

In Section 3 we show a satisfactory small time existence result (Theorems 3.1, 3.22 and Remark 3.24) of a smooth motion for a special class of networks, that is, the ones having only multi-points with three concurrent curves forming angles of 120 degrees (this last property is called Herring condition).

We have to say that the uniqueness problem is less clear at the moment (see Remark 3.23).

In the case of the presence in the initial network of a "bad" 3-point, not satisfying the Herring condition, we are not able at the moment to show the existence of a flow, smooth for every positive time, satisfying a "robust" definition (at least as Definition 2.8).

Actually, one would expect that the desired good definition should give uniqueness of the motion and force, by an instantaneous regularization, the three angles to become immediately of 120 degrees and to remain so. This is sustained by the fact that, by an energy argument ([10]), any smooth Brakke flow has to share such a property (which is also suggested by numerical and physical experiments, see at http://mimp.mems.cmu.edu and also the discussions in [9], [10], [11], [20], [30]).

Notice that, by the variational nature of the problem it is appealing to guess that some sort of parabolic regularization could play a role here. We remark that if a multi-point has only two concurrent curves, it can be shown, by the 
regularizing effect of the evolution by curvature (see [4], [6], [7], [19]), that the two curves together become instantaneously a single smooth curve moving by curvature. Hence, the 2-point has vanished but this particular event is so "soft" (and topologically null) that we can avoid to consider it as a real structural change.

We discuss now some other difficulties of geometric character in having a good definition of flow for a generic network.

1. The presence of multi-points $O^{j}$ of order greater than three:

In the case of a 4-point (and clearly also of a higher order multi-point), for instance, considering the network described by two curves crossing each other, there are really several possible candidates for the flow, even excluding a priori "fattening" phenomena. One cannot easily decide how the angles must behave, like in the 3-point case above, moreover, one can allow the four concurrent curves to separate in two pairs of curves moving independently each other and it could even be taken into account the "creation" of new multi-points from such a single one (all these events are actually possible in Brakke's definition).

In these latter cases, the topology of the network changes dramatically, forcing us to change the structure of the system of equations governing the evolution and the family of curves composing the network.

2. The presence of several multi-points $O^{j}$ :

during the flow some of them can "collapse" together, again modifying the topological structure of the network, when the length of at least one curve of the network goes to zero (which can actually happen). In this case, like at the previous point, one possibly has to "restart" the evolution with a different set of curves.

Notice that even if one starts with a network such that all the multipoints are 3-points, in the event of a collapse, one could have to face a situation with multi-points of order higher than three (consider for instance two 3-points collapsing along a single curve connecting them) or to deal with "bad" 3-points (think of three 3-points collapsing together along three curves connecting them).

REMARK 1.2. Actually, it seems reasonable that the configurations with multi-points of order greater than three or 3-points with angles different from 120 degrees should be unstable (actually, they are unstable for the Length functional), with the meaning that they can appear at some discrete set of times (and probably in some cases are unavoidable), but they must vanish immediately after.

Because of all these complications, as a first step, in this paper we are going to analyze the simplest possible network which, by construction, rules out all the troubles related to the cases above.

Anyway, we will point out when the results can be extended to more general networks. 
Definition 1.3. We call triod $\mathbb{T}$ in $\Omega$ a special network composed only of three regular, embedded $C^{2}$ curves $\sigma^{i}:[0,1] \rightarrow \bar{\Omega}$ (here regular means $\sigma_{x}^{i}(x) \neq 0$ for every $x \in[0,1]$ and $\left.i \in\{1,2,3\}\right)$, where $\Omega$ is a smooth open convex subset of $\mathbb{R}^{2}$, moreover, these curves (sometimes we will call them also edges of the triod) intersect each other only at a single 3-point $O=\sigma^{1}(0)=$ $\sigma^{2}(0)=\sigma^{3}(0)$ (the 3-point of the triod $\mathbb{T}$ ) and have the other three end points coinciding with three distinct points $P^{i}=\sigma^{i}(1)$ (the end points of $\mathbb{T}$ ) belonging to the boundary of $\Omega$.

Finally, we assume that the tangents of the three curves form angles of 120 degrees at the 3-point $O$.

We say that the triod is (of class) $C^{k}$ or $C^{\infty}$ if the three curves are respectively $C^{k}$ or $C^{\infty}$. We remark that with this definition we assume that, unless explicitly otherwise stated, every triod is at least of class $C^{2}$, in order to speak of its curvature.

Notice that the angular condition can be expressed as

$$
\sum_{i=1}^{3} \frac{\sigma_{x}^{i}(0)}{\left|\sigma_{x}^{i}(0)\right|}=0 .
$$

It is obvious that during the motion of such a triod the "bad" configurations we discussed before are a priori excluded.

REMARK 1.4. Since in all the paper we will consider only triods with angles of 120 degrees between the curves, for sake of simplicity, we chose to call these sets simply triods with the meaning "triods with angles of 120 degrees".

Moreover, sometimes we will speak of a triod without end points composed of three curves $\sigma^{i}:[0,+\infty) \rightarrow \mathbb{R}^{2}$, for instance, when we will need to allow them go to infinity.

Actually, we could also consider the possibility that the three curves intersect each other, so when ambiguity is possible, we will underline the property of non self-intersection saying that the triod is embedded.

The paper is devoted to study the existence, uniqueness, regularity and asymptotic behavior of these embedded triods in $\Omega$, moving by curvature keeping fixed the end points $P^{1}, P^{2}, P^{3}$.

This problem has been considered by Bronsard and Reitich in [11], where they prove an existence result which is the core of Theorem 3.1 and by Kinderlehrer and Liu in [30] showing the global existence of a flow for an initial triod sufficiently close to the minimal configuration connecting the three points $P^{i}$ (Steiner configuration).

We want to extend all this to any embedded initial triod, concentrating in particular on the global existence and regularity of the flow. Even if this is the simplest case, its understanding is clearly crucial in analyzing more general networks, taking also into account Remark 1.2.

Our conjecture is that any embedded initial triod evolves in time without singularities and asymptotically converges to the minimal connection between the three 
points $P^{i}$ if the lengths of the three curves stay away from zero, being the "vanishing" of a curve the only possible "catastrophic" event during the flow.

REMARK 1.5. It should be noticed that if the triangle formed by the points $P^{1}, P^{2}, P^{3}$ has an angle of more than 120 degrees, then a triod composed of three segments forming angles of 120 and connecting its vertices does not exist. Then, if the conjecture is true, necessarily one of the lengths is not uniformly bounded away from zero.

After discussing the existence (and uniqueness) of a smooth flow on some maximal time interval in the first part of the paper, in order to prove such conjecture we try to generalize the analysis of the motion by mean curvature of smooth closed curves and hypersurfaces in the Euclidean space, employing a mix of PDE's and differential geometry techniques.

Essentially, what is needed is the understanding of the structure of the possible blow up around the singularities, in order to actually exclude these latter by means of geometric arguments. Some key references for this line of research are [5], [22], [25], [26].

The most relevant difference between our case and the smooth one, is the difficulty in using the maximum principle, which is the main tool to get estimates on the geometric quantities during the flow. Indeed, the 3-point (by the 120 degrees condition) is nice from the distributional point of view: in a sense, it is an interior point, but it is troublesome for any argument based on the maximum principle, since it behaves like a boundary point.

For this reason, some important pointwise estimates which are almost trivial applications of the maximum principle in the smooth case, are here much more complicated to prove (sometimes we do not even know if they actually hold) and we will have to resort to integral estimates. These latter are similar to the ones in [5], [6], [7], [24] for instance, but require some extra work in order to deal with this strange "boundary" point.

If the lengths of the curves do not reduce to zero, by means of these latters estimates, we can see that at the time $T$ of singularity the curvature has to explode, then like in the smooth case, we separate the analysis according to its rate of blow up.

We say that a singularity is of Type $I$ if for some constant $C$ we have $\max _{\mathbb{T}_{t}} k^{2} \leq C /(T-t)$ as $t \rightarrow T$ and it is of Type II otherwise.

Rescaling properly the flow around a hypothetical Type I singularity one gets an evolution of embedded triods (unbounded and without end points) shrinking homothetically during the motion by curvature. Classifying all such particular evolutions, we will show that none of them can arise as a blow up of the flow $\mathbb{T}_{t}$, this clearly implies that Type I singularities cannot develop.

With the same idea, rescaling the flow around a Type II singularity, one gets an eternal motion by curvature, that is, an evolution of triods defined for every time $t \in \mathbb{R}$.

What is missing at the moment is that this eternal flow is actually simply given by a translating triod (unbounded and without end points), like it happens 
in the case of a single smooth curve. Here also, the main difficulty resides in replacing some maximum principle arguments.

If the blow up would be translating, after classification, we could exclude also this case by means of an argument based on the monotonicity of a geometric quantity (see Sections 5 and 7 for details), hence, no singularity at all could appear during the flow if the lengths of the three curves of the triod stay away from zero.

The conjecture then would follow.

Acknowledgment. We are grateful to Alessandra Lunardi for helping us in the proof of the small time existence of a smooth flow in Section 3.

\section{2. - Definitions and preliminaries}

We start setting in a precise analytical way the curvature evolution problem for an embedded initial triod $\mathbb{T}_{0}=\cup_{i=1}^{3} \sigma^{i}$ in $\Omega$.

Definition 2.1. We say that the triods $\mathbb{T}_{t}=\cup_{i=1}^{3} \gamma^{i}(\cdot, t)$ evolve by curvature (remaining embedded) in the time interval $[0, T)$ if the three functions $\gamma^{i}$ : $[0,1] \times[0, T) \rightarrow \bar{\Omega}$ are of class $C^{2}$ in space and $C^{1}$ in time, at least, and satisfy the following quasilinear parabolic system

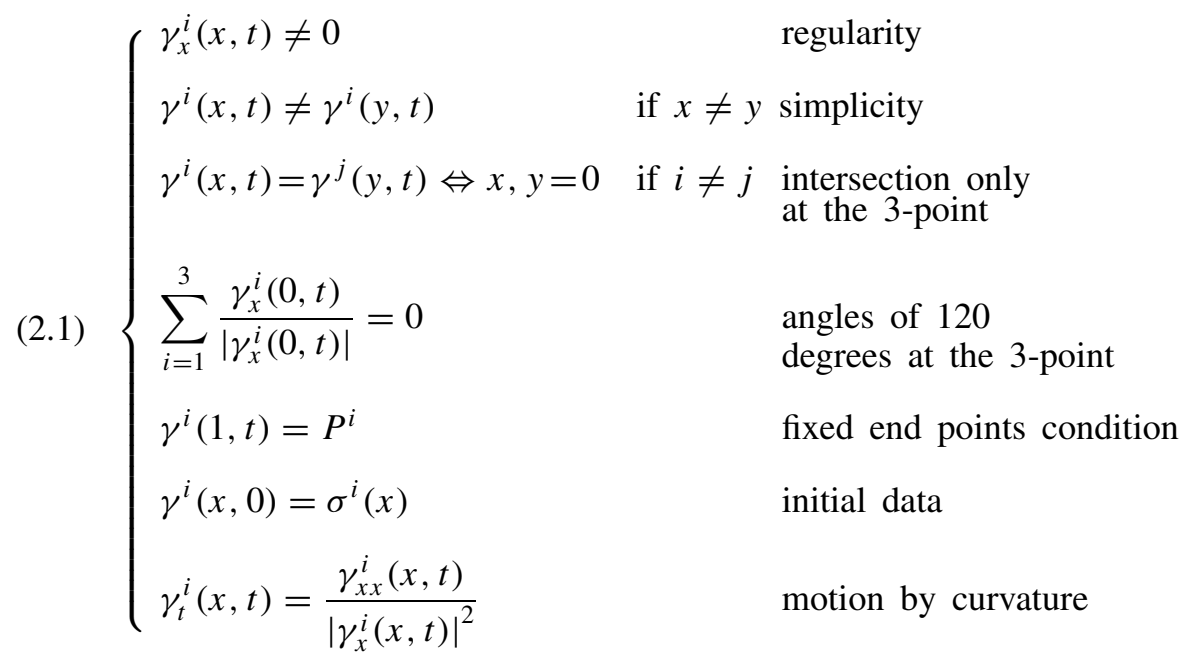

for every $x \in[0,1], t \in[0, T)$ and $i, j \in\{1,2,3\}$. 
REMARK 2.2. We spend some words on the evolution equation

$$
\gamma_{t}^{i}=\frac{\gamma_{x x}^{i}}{\left|\gamma_{x}^{i}\right|^{2}},
$$

which is not the usual way to describe the motion by curvature, that is,

$$
\gamma_{t}^{i}=\frac{\left\langle\gamma_{x x}^{i} \mid v^{i}\right\rangle}{\left|\gamma_{x}^{i}\right|^{2}} v^{i}=k^{i} v^{i}
$$

where we denoted with $v^{i}$ the unit normal to the curve $\gamma^{i}$ and $k^{i}$ its curvature.

The two velocities differ only by a tangential component which actually affects the motions of the single points (Lagrangian point of view), but it does not affect the local motion of a curve as a whole subset of $\mathbb{R}^{2}$ (Eulerian point of view).

We remark that this property of global invariance is not peculiar to this particular tangential term, it holds for any tangential modification of the velocity. This fact is well known for the curvature evolution of a smooth curve, hence also for a triod, any tangential contribution to the velocity does not modify the flow outside the 3-point.

In our situation such extra term becomes necessary in order to allow the motion of the 3-point $O(t)=\gamma^{i}(0, t)$. Indeed, since we look for a $C^{2}$ solution of Problem (2.1), if the velocity would be in normal direction at every point of the three curves, the 3-point should move in a direction which is normal to all of them, then the only possibility would be that it does not move at all (see also the discussions and examples in [10], [11], [30]).

REMARK 2.3. It should be noticed that this definition of flow of a $C^{2}$ triod is very strong, indeed, as the maps $\gamma^{i}$ have to be $C^{1}$ in time and $C^{2}$ is space till the parabolic boundary, the compatibility conditions of order 2 have to be satisfied, that is,

$$
\begin{aligned}
& \frac{\sigma_{x x}^{i}(1)}{\left|\sigma_{x}^{i}(1)\right|^{2}}=0 \text { for every } i \in\{1,2,3\} \text { and } \\
& \frac{\sigma_{x x}^{i}(0)}{\left|\sigma_{x}^{i}(0)\right|^{2}}=\frac{\sigma_{x x}^{j}(0)}{\left|\sigma_{x}^{j}(0)\right|^{2}} \text { for every } i, j \in\{1,2,3\} .
\end{aligned}
$$

(the compatibility conditions of order 0 and 1 are automatically satisfied, since they are equivalent to say that the three curves $\sigma^{i}$ form a 3-point with angles of 120 degrees, which is assumed by construction).

This means, for instance, that for the initial triod the curvature at the end points $P^{i}$ and the sum of the three curvatures at the 3-point have to be zero (see later).

Notice that these two conditions on $\mathbb{T}_{0}$ are really geometric, independent of the parametrization of the curves $\gamma^{i}$, but intrinsic to the set $\mathbb{T}_{0}$ (see Definition 2.5 and Remark 2.7) and are not satisfied by a generic $C^{2}$ triod. 
Since we are interested in an existence theorem for any triod composed of $C^{2}$ curves (Theorem 3.22), we consider also Brakke's definition of curvature flow.

Definition 2.4. We say that a triod $\mathbb{T}_{0}$, composed of three $C^{\infty}$ curves $\sigma^{i}$ is smooth if it satisfies the compatibility conditions of every order of parabolic system (2.1) (this is clearly much more striking that being simply a $C^{\infty}$ triod according to Definition 1.3).

Precisely, this means that at the end points and at the 3-point, there hold all the relations on the space derivatives of the functions $\sigma^{i}$ obtained differentiating in time, at $t=0$, the boundary conditions.

We say that a solution $\mathbb{T}_{t}$ of Problem (2.1), given by the curves $\gamma^{i}(x, t) \in$ $C^{\infty}([0,1] \times[0, T))$ is a smooth flow if the compatibility conditions of every order are satisfied at every time $t \in[0, T)$, that is, all the triods $\mathbb{T}_{t}$ are smooth.

Definition 2.5. We say that a triod $\mathbb{T}_{0}$ is geometrically smooth if there exist a regular parametrization of its three curves such that the resulting triod is smooth.

To denote a flow we will often write simply $\mathbb{T}_{t}$ instead of letting explicit the curves $\gamma^{i}$ which compose the triods.

Moreover, it will be also useful to describe a triod as a map $F: \mathbb{T} \rightarrow \bar{\Omega}$ from a fixed standard triod $\mathbb{T}$ in $\mathbb{R}^{2}$, composed of three unit segments from the origin in the plane, forming angles of 120 degrees. In this case we will still denote with $O$ the 3-point of $\mathbb{T}$ and with $P^{i}$ the three end points of such standard triod.

The evolution then will be given by a map $F: \mathbb{T} \times[0, T) \rightarrow \bar{\Omega}$, constructed naturally from the curves $\gamma^{i}$, so $\mathbb{T}_{t}=F(\mathbb{T}, t)$.

We adopt the following notation:

$$
\begin{array}{rlrl}
\tau^{i} & =\tau^{i}(x, t)=\frac{\gamma_{x}^{i}}{\left|\gamma_{x}^{i}\right|} & & \text { unit tangent vector to } \gamma^{i}, \\
v^{i} & =v^{i}(x, t)=\mathrm{R} \tau^{i}(x, t)=\mathrm{R} \frac{\gamma_{x}^{i}}{\left|\gamma_{x}^{i}\right|} & & \text { unit normal vector to } \gamma^{i}, \\
O & =O(t)=\gamma^{i}(0, t) & & \text { 3-point of the triod } \mathbb{T}_{t}, \\
\underline{v}^{i} & =\underline{v}^{i}(x, t)=\frac{\gamma_{x x}^{i}}{\left|\gamma_{x}^{i}\right|^{2}} & & \text { velocity of the point } \gamma^{i}(x, t), \\
\lambda^{i} & =\lambda^{i}(x, t)=\frac{\left\langle\gamma_{x x}^{i} \mid \tau^{i}\right\rangle}{\left|\gamma_{x}^{i}\right|^{2}}=\frac{\left\langle\gamma_{x x}^{i} \mid \gamma_{x}^{i}\right\rangle}{\left|\gamma_{x}^{i}\right|^{3}} & & \text { tangential velocity of the point } \gamma^{i}(x, t), \\
k^{i} & =k^{i}(x, t)=\frac{\left\langle\gamma_{x x}^{i} \mid v^{i}\right\rangle}{\left|\gamma_{x}^{i}\right|^{2}}=\left\langle\partial_{s} \tau^{i} \mid v^{i}\right\rangle= & \\
& =-\left\langle\partial_{s} v^{i} \mid \tau^{i}\right\rangle & & \text { curvature at the point } \gamma^{i}(x, t),
\end{array}
$$


where $s$ is the arclength parameter on the relative curve, defined by $s(x, t)=$ $\int_{0}^{x}\left|\gamma_{x}^{i}(\xi, t)\right| d \xi$, and $\mathrm{R}: \mathbb{R}^{2} \rightarrow \mathbb{R}^{2}$ is the counterclockwise rotation of $\pi / 2$. Notice that $\partial_{s}=\left|\gamma_{x}^{i}\right|^{-1} \partial_{x}$.

Moreover, we set $\underline{\lambda}^{i}=\lambda^{i} \tau^{i}$ and $\underline{k}^{i}=k^{i} v^{i}$, then, it clearly follows that $\underline{v}^{i}=\underline{\lambda}^{i}+\underline{k}^{i}$ and $\left|\underline{v}^{i}\right|^{2}=\left(\lambda^{i}\right)^{2}+\left(k^{i}\right)^{2}$.

Here and in the sequel, we denote with $\partial_{s} f$ and $\partial_{t} f$ the derivatives of a function $f$ along a curve with respect to the relative arclength parameter and the time, $\partial_{s}^{n} f, \partial_{t}^{n} f$ are the higher order partial derivatives which often we will also write as $f_{s}, f_{s s}, \ldots$ and $f_{t}, f_{t t}, \ldots$

We adopt the following convention for integrals,

$$
\begin{aligned}
\int_{\mathbb{T}_{t}} f & \left(t, \gamma, \tau, \nu, k, k_{s}, \ldots, \lambda, \lambda_{s} \ldots\right) d s \\
& =\sum_{i=1}^{3} \int_{0}^{1} f\left(t, \gamma^{i}, \tau^{i}, v^{i}, k^{i}, k_{s}^{i}, \ldots, \lambda^{i}, \lambda_{s}^{i} \ldots\right)\left|\gamma_{x}^{i}\right| d x
\end{aligned}
$$

as the arclength measure is given by $d s=\left|\gamma_{x}^{i}\right| d x$ on the curve $\gamma^{i}$.

In general, if there is no need to make explicit the three curves composing a triod, we simply write $\tau, v, \underline{v}, \lambda$ and $k$ for the previous quantities, omitting the indices.

We suppose now to have a smooth flow $\mathbb{T}_{t}$ on some positive time interval $[0, T)$ and we write the evolution equations for the geometric quantities.

LEMMA 2.6. If $\gamma$ is a curve moving by

$$
\gamma_{t}=\frac{\gamma_{x x}}{\left|\gamma_{x}\right|^{2}}=\lambda \tau+k \nu
$$

then the following commutation rule holds,

$$
\partial_{t} \partial_{s}=\partial_{s} \partial_{t}+\left(k^{2}-\lambda_{s}\right) \partial_{s}
$$

Proof. Let $f:[0,1] \times[0, T) \rightarrow \mathbb{R}$ be a smooth function, then

$$
\begin{aligned}
\partial_{t} \partial_{s} f-\partial_{s} \partial_{t} f & =\frac{f_{t x}}{\left|\gamma_{x}\right|}-\frac{\left\langle\gamma_{x} \mid \gamma_{x t}\right\rangle f_{x}}{\left|\gamma_{x}\right|^{3}}-\frac{f_{t x}}{\left|\gamma_{x}\right|}=-\left\langle\tau \mid \partial_{s} \gamma_{t}\right\rangle \partial_{s} f \\
& =-\left\langle\tau \mid \partial_{s}(\lambda \tau+k \nu)\right\rangle \partial_{s} f=\left(k^{2}-\lambda_{s}\right) \partial_{s} f
\end{aligned}
$$

and the formula is proved. 
Then we can compute,

$$
\begin{aligned}
\partial_{t} \tau & =\partial_{t} \partial_{s} \gamma=\partial_{s} \partial_{t} \gamma+\left(k^{2}-\lambda_{s}\right) \partial_{s} \gamma=\partial_{s}(\lambda \tau+k v)+\left(k^{2}-\lambda_{s}\right) \tau \\
& =\left(k_{s}+k \lambda\right) v \\
\partial_{t} \nu & =\partial_{t}(\mathrm{R} \tau)=\mathrm{R} \partial_{t} \tau=-\left(k_{s}+k \lambda\right) \tau \\
\partial_{t} k & =\partial_{t}\left\langle\partial_{s} \tau \mid v\right\rangle=\left\langle\partial_{t} \partial_{s} \tau \mid \nu\right\rangle=\left\langle\partial_{s} \partial_{t} \tau \mid \nu\right\rangle+\left(k^{2}-\lambda_{s}\right)\left\langle\partial_{s} \tau \mid \nu\right\rangle \\
& =\partial_{s}\left\langle\partial_{t} \tau \mid \nu\right\rangle+k^{3}-k \lambda_{s}=\partial_{s}\left(k_{s}+k \lambda\right)+k^{3}-k \lambda_{s} \\
& =k_{s s}+k_{s} \lambda+k^{3} \\
\partial_{t} \lambda & =-\partial_{t} \partial_{x} \frac{1}{\left|\gamma_{x}\right|}=\partial_{x} \frac{\left\langle\gamma_{x} \mid \gamma_{t x}\right\rangle}{\left|\gamma_{x}\right|^{3}}=\partial_{x} \frac{\left\langle\tau \mid \partial_{s}(\lambda \tau+k \nu)\right\rangle}{\left|\gamma_{x}\right|}=\partial_{x} \frac{\left(\lambda_{s}-k^{2}\right)}{\left|\gamma_{x}\right|} \\
& =\partial_{s}\left(\lambda_{s}-k^{2}\right)-\lambda\left(\lambda_{s}-k^{2}\right)=\lambda_{s s}-\lambda \lambda_{s}-2 k k_{s}+\lambda k^{2} .
\end{aligned}
$$

As the triods $\mathbb{T}_{t}$ are smooth, differentiating in time the concurrency condition $\gamma^{i}(0, t)=\gamma^{j}(0, t)$ we obtain $\lambda^{i} \tau^{i}+k^{i} v^{i}=\lambda^{j} \tau^{j}+k^{j} v^{j}$ at the 3-point for every pair of indices $i, j$. Multiplying these vector equalities for $\tau^{l}$ and $v^{l}$ and varying $i, j, l$ we get the relations

$$
\begin{aligned}
& \lambda^{i}=-\lambda^{i+1} / 2-\sqrt{3} k^{i+1} / 2 \\
& \lambda^{i}=-\lambda^{i-1} / 2+\sqrt{3} k^{i-1} / 2 \\
& k^{i}=-k^{i+1} / 2+\sqrt{3} \lambda^{i+1} / 2 \\
& k^{i}=-k^{i-1} / 2-\sqrt{3} \lambda^{i-1} / 2
\end{aligned}
$$

with the convention that the superscripts are considered modulus three. Solving this system we get

$$
\begin{aligned}
\lambda^{i} & =\frac{k^{i-1}-k^{i+1}}{\sqrt{3}} \\
k^{i} & =\frac{\lambda^{i+1}-\lambda^{i-1}}{\sqrt{3}}
\end{aligned}
$$

which implies

$$
\sum_{i=1}^{3} k^{i}=\sum_{i=1}^{3} \lambda^{i}=0
$$

at the 3-point of the triods $\mathbb{T}_{t}$. Moreover, considering $\mathrm{K}=\left(k^{1}, k^{2}, k^{3}\right)$ and $\Lambda=\left(\lambda^{1}, \lambda^{2}, \lambda^{3}\right)$ as vectors in $\mathbb{R}^{3}$, we have seen that $\mathrm{K}$ and $\Lambda$ belong to the plane orthogonal to the vector $(1,1,1)$ and $\mathrm{K}=\Lambda \wedge(1,1,1) / \sqrt{3}, \Lambda=$ $-\mathrm{K} \wedge(1,1,1) / \sqrt{3}$ that is, $\mathrm{K}=\mathrm{S} \Lambda$ and $\Lambda=-\mathrm{SK}$ where $\mathrm{S}$ is the rotation in 
$\mathbb{R}^{3}$ of an angle of $\pi / 2$ around the axis $I=\langle(1,1,1)\rangle$. Hence, it also follows that

$$
\sum_{i=1}^{3}\left(k^{i}\right)^{2}=\sum_{i=1}^{3}\left(\lambda^{i}\right)^{2} \quad \text { and } \quad \sum_{i=1}^{3} k^{i} \lambda^{i}=0 .
$$

at the 3-point of the triods $\mathbb{T}_{t}$.

Now we differentiate in time the angular condition $\sum_{i=1}^{3} \tau^{i}(0, t)=0$ at the 3-point, by equation (2.4) we get

$$
k_{s}^{i}+\lambda^{i} k^{i}=k_{s}^{j}+\lambda^{j} k^{j}
$$

for every pair $i, j$. In terms of vectors $\mathbb{R}^{3}$ as before, we can write

$$
\mathrm{K}_{s}+\Lambda \mathrm{K}=\left(k_{s}^{1}+\lambda^{1} k^{1}, k_{s}^{2}+\lambda^{2} k^{2}, k_{s}^{3}+\lambda^{3} k^{3}\right) \in \mathrm{I} .
$$

Differentiating repeatedly in time all these vector relations we have

$$
\begin{gathered}
\partial_{t}^{l} \mathrm{~K}, \partial_{t}^{l} \Lambda \perp \mathrm{I} \text { and } \partial_{t}^{l}(\mathrm{~K} \mathbb{A})=0 \\
\partial_{t}^{l} \Lambda=-\partial_{t}^{l} \mathrm{SK}=-\mathrm{S} \partial_{t}^{l} \mathrm{~K} \\
\partial_{t}^{m}\left(\mathrm{~K}_{s}+\Lambda \mathrm{K}\right) \in \mathrm{I}
\end{gathered}
$$

which, making explicit the indices, give the following equalities at the 3-point,

$$
\begin{gathered}
\partial_{t}^{l} \sum_{i=1}^{3} k^{i}=\sum_{i=1}^{3} \partial_{t}^{l} k^{i}=\partial_{t}^{l} \sum_{i=1}^{3} \lambda^{i}=\sum_{i=1}^{3} \partial_{t}^{l} \lambda^{i}=\partial_{t} \sum_{i=1}^{3} k^{i} \lambda^{i}=0, \\
\sum_{i=1}^{3}\left(\partial_{t}^{l} k^{i}\right)^{2}=\sum_{i=1}^{3}\left(\partial_{t}^{l} \lambda^{i}\right)^{2} \text { for every } l \in \mathbb{N}, \\
\partial_{t}^{m}\left(k_{s}^{i}+\lambda^{i} k^{i}\right)=\partial_{t}^{m}\left(k_{s}^{j}+\lambda^{j} k^{j}\right) \text { for every pair } i, j \text { and } m \in \mathbb{N} .
\end{gathered}
$$

By the orthogonality relations with respect to the axis I, we get also

$$
\partial_{t}^{l} K \partial_{t}^{m}\left(\mathrm{~K}_{s}+\Lambda \mathrm{K}\right)=\partial_{t}^{l} \Lambda \partial_{t}^{m}\left(\mathrm{~K}_{s}+\Lambda \mathrm{K}\right)=0,
$$

that is,

(2.10) $\sum_{i=1}^{3} \partial_{t}^{l} k^{i} \partial_{t}^{m}\left(k_{s}^{i}+\lambda^{i} k^{i}\right)=\sum_{i=1}^{3} \partial_{t}^{l} \lambda^{i} \partial_{t}^{m}\left(k_{s}^{i}+\lambda^{i} k^{i}\right)=0$ for every $l, m \in \mathbb{N}$.

Looking then at the three end points, by Lemma 3.10 in the next section, we have that all the even space derivatives of $k$ and $\lambda$ are zero. 
REMARK 2.7. As the "shape" of the curves is fixed under reparametrization, if a triod is geometrically smooth, the three curves have to be $C^{\infty}$ once parametrized in arclength. Moreover, necessarily $\sum_{i=1}^{3} v^{i}=0$, and $\sum_{i=1}^{3} k^{i}=0$ at the 3-point must hold, like all the relations one gets from these two, iteratively, differentiating in time by means of formulas (2.5), (2.6) and substituting every occurrence of $\lambda^{i}$ according to the formula $\left(\lambda^{1}, \lambda^{2}, \lambda^{3}\right)=-\left(k^{1}, k^{2}, k^{3}\right) \wedge$ $(1,1,1) / \sqrt{3}$ (notice that in this way $\lambda_{s}$ never appears). Working analogously at the end points, the compatibility conditions reduce to require that every even space derivative of the curvature is zero (by Lemma 3.10 in the next section).

These necessary conditions are actually also sufficient, indeed the geometrical smoothness is a matter of curvature, not involving $\lambda$. If a triod satisfies such conditions, we can parametrize every curve $\gamma^{i}$ in a way that $\lambda^{i}$ at the 3 -point has the right value given by the relation $\lambda^{i}=-(\mathrm{K} \wedge \mathrm{I})^{i} / \sqrt{3}$ and it is zero at the end point of the curve, for instance, setting $\lambda_{s}^{i}$ constantly equal to $-\lambda^{i}(0) / L^{i}$, where $L^{i}$ is the length of the curve.

It is straightforward to check that this can be done and the resulting parametrization gives a smooth triod.

Definition 2.8. We will speak of Brakke flow with equality of an initial triod $\mathbb{T}_{0}$ in $[0, T)$, for a family of $C^{2}$ triods $\mathbb{T}_{t}$ in $\Omega$ all with the same end points as $\mathbb{T}_{0}$ and satisfying the equation

$$
\frac{d}{d t} \int_{\mathbb{T}_{t}} \varphi(\gamma, t) d s=-\int_{\mathbb{T}_{t}} \varphi(\gamma, t) k^{2} d s+\int_{\mathbb{T}_{t}}\langle\nabla \varphi(\gamma, t) \mid \underline{k}\rangle d s+\int_{\mathbb{T}_{t}} \varphi_{t}(\gamma, t) d s,
$$

for every smooth function with compact support $\varphi: \Omega \times[0, T) \rightarrow \mathbb{R}$ and $t \in[0, T)$.

This means also that the time derivative at the left hand side has to exist. The right hand side does not give any problem since the triods are $C^{2}$, at least.

We will say that a Brakke flow is smooth if all the triods are geometrically smooth.

REMARK 2.9. It is straightforward to check that a solution of Problem 2.1 is also a smooth Brakke flow with equality.

Actually, the original definition of Brakke flow stated in [10, Section 3.3] allows equality (2.11) to be an inequality (and triods $\mathbb{T}_{t}$ to be one-dimensional countably rectifiable subsets of $\mathbb{R}^{2}$ with a distributional notion of curvature, called varifolds, see [34]), precisely,

$$
\begin{aligned}
\frac{\bar{d}}{d t} \int_{\mathbb{T}_{t}} \varphi(x, t) d \mathcal{H}^{1}(x) \leq & -\int_{\mathbb{T}_{t}} \varphi(x, t) k^{2} d \mathcal{H}^{1}(x) \\
& +\int_{\mathbb{T}_{t}}\langle\nabla \varphi(x, t) \mid \underline{k}\rangle d \mathcal{H}^{1}(x)+\int_{\mathbb{T}_{t}} \varphi_{t}(x, t) d \mathcal{H}^{1}(x),
\end{aligned}
$$

must hold for every positive smooth function with compact support $\varphi: \Omega \times$ $[0, T) \rightarrow \mathbb{R}$ and $t \in[0, T)$, where $\frac{\bar{d}}{d t}$ is the upper derivative (the $\overline{\lim }$ of the 
incremental ratios) and $\mathcal{H}^{1}$ is the Hausdorff one-dimensional measure in $\mathbb{R}^{2}$ (we will use this notation through all the paper).

This weaker condition was introduced by Brakke in order to prove an existence result [10, Section 4.13] for a family of initial sets much wider than the networks of curves, but, on the other hand, it let open the possibility of instantaneous vanishing of some parts of the set.

Since for our triods (probably, this can be done also for a general network) we are able to show the existence of a Brakke flow via a different method, and this flow is composed of smooth triods and satisfies the equality, for sake of simplicity, we included such extra properties in the definition.

A big difference between Brakke flows and the evolutions obtained as solutions of Problem (2.1) is that the former triods are simply considered as sets without any mention to their parametrization (that clearly is not unique). This means that actually a Brakke flow can be a family of triods given by the maps $\gamma^{i}(x, t)$ which are $C^{2}$ in space, but possibly do not have absolutely any regularity with respect to the time variable $t$.

If we consider two different smooth triods $\mathbb{T}_{0}^{1}$ and $\mathbb{T}_{0}^{2}$ which are the same subset of $\mathbb{R}^{2}$, giving two different solutions $\mathbb{T}_{t}^{1}$ and $\mathbb{T}_{t}^{2}$ of Problem (2.1) on some common interval, then the two associated Brakke flows coincide (Proposition 3.3 ), that is, as subsets of $\mathbb{R}^{2}$, forgetting the parametrization, actually $\mathbb{T}_{t}^{1}=\mathbb{T}_{t}^{2}$ for every time $t$.

This means that the geometric evolution problem has a satisfactory uniqueness property if the initial triod is smooth.

In general, when we will speak of geometric problem we will mean that we are thinking of the triods as subsets of $\mathbb{R}^{2}$, independently of the parametrizations of their curves.

An open question is whether any smooth Brakke flow with equality admits a parametrization of the initial triod with an associate solution of Problem (2.1) representing it at least for some time.

A positive answer would imply the uniqueness in the class of these special Brakke flows and the coincidence of the two formulations, from the geometric point of view.

Finally we state precisely the conjecture which is the main topic of the second part of the paper.

Here and in the following, we denote with $L^{i}$ the lengths of the three curves and with $L=L^{1}+L^{2}+L^{3}$ the total length of the triod.

Conjecture 2.10. Let $\mathbb{T}_{t}$ be a smooth evolution of embedded triods on a maximal time interval $[0, T)$.

If $\underline{\lim }_{t \rightarrow T} L^{i} \neq 0$ for every $i \in\{1,2,3\}$, then $T=+\infty$ and $\mathbb{T}_{t}$ converges, as $t \rightarrow+\infty$, to the minimal connection between the three points $P^{i}$. 


\section{3. - Small time existence and a priori estimates}

The first small time existence result for a flow very similar to Problem (2.1) is due to Bronsard and Reitich [11]. In their paper it is shown the existence of a unique solution $\gamma^{i} \in C^{2+2 \alpha, 1+\alpha}([0,1] \times[0, T])$ of the same parabolic system, for an initial triod composed of three curves $\sigma^{i} \in C^{2+2 \alpha}([0,1])$ and satisfying the natural compatibility conditions. The only difference is that they impose the Neumann boundary condition of orthogonal intersection with $\partial \Omega$, instead of keeping the end points $P^{i} \in \partial \Omega$ fixed as we do.

The same technique works also in our case and gives the small time existence of a unique solution $\gamma^{i} \in C^{2+2 \alpha, 1+\alpha}([0,1] \times[0, T])$ (notice that this means that the curves are $C^{2+2 \alpha}$ till the 3-point and their end points $P^{i}$ ) of the following parabolic system

$$
\begin{cases}\gamma_{x}^{i}(x, t) \neq 0 & \text { regularity } \\ \gamma^{i}(0, t)=\gamma^{j}(0, t) & \text { concurrence at the 3-point } \\ \sum_{i=1}^{3} \frac{\gamma_{x}^{i}(0, t)}{\left|\gamma_{x}^{i}(0, t)\right|}=0 & \text { angles of } 120 \text { degrees at the 3-point } \\ \gamma^{i}(1, t)=P^{i} & \text { fixed end points condition } \\ \gamma^{i}(x, 0)=\sigma^{i}(x) & \text { initial data } \\ \gamma_{t}^{i}(x, t)=\frac{\gamma_{x x}^{i}(x, t)}{\left|\gamma_{x}^{i}(x, t)\right|^{2}} & \text { motion by curvature }\end{cases}
$$

given any initial $C^{2+2 \alpha}$ triod $\mathbb{T}_{0}=\cup_{i=1}^{3} \sigma^{i}$, with $\alpha \in(0,1 / 2)$, satisfying the compatibility conditions of order 2 , that is,

$$
\begin{aligned}
& \frac{\sigma_{x x}^{i}(1)}{\left|\sigma_{x}^{i}(1)\right|^{2}}=0 \text { for every } i \in\{1,2,3\} \text { and } \\
& \frac{\sigma_{x x}^{i}(0)}{\left|\sigma_{x}^{i}(0)\right|^{2}}=\frac{\sigma_{x x}^{j}(0)}{\left|\sigma_{x}^{j}(0)\right|^{2}} \text { for every } i, j \in\{1,2,3\}
\end{aligned}
$$

(the compatibility conditions of order 0 and 1 are automatically satisfied, since they are equivalent to say that the three curves $\sigma^{i}$ form a triod with angles of 120 degrees).

Now we look for a higher regularity result.

THEOREM 3.1. For any initial smooth triod $\mathbb{T}_{0}$ there exists a unique smooth solution of Problem (2.1) on a maximal time interval $[0, T)$.

Proof. Since the initial triod $\mathbb{T}_{0}$ satisfies the compatibility conditions at every order, the method of Bronsard and Reitich actually provides a way, for every $n \in \mathbb{N}$, to get a unique solution in $C^{2 n+2 \alpha, n+\alpha}\left([0,1] \times\left[0, T_{n}\right]\right)$, satisfying the compatibility conditions of order $0, \ldots, n$ at every time. 
Then, by standard methods of one-dimensional parabolic equations we can obtain a solution which belongs to $C^{\infty}([0,1] \times[0, T))$ for some small positive time $T>0$ and consider its maximal time of existence.

We give just the line of the proof and we indicate the relevant references for the details.

Let us consider a solution $\gamma^{i} \in C^{2 n+2 \alpha, n+\alpha}\left([0,1] \times\left[0, T_{n}\right]\right)$ for $n \geq 2$, then the functions $\gamma_{x}^{i}(x, t)$ belong to $C^{2 n-1+2 \alpha, n-1 / 2+\alpha}\left([0,1] \times\left[0, T_{n}\right]\right.$ ) (see for instance [32, Lemma 5.1.1]), then we look at the parabolic system satisfied by $v^{i}(x, t)=\gamma_{t}^{i}(x, t)$,

$$
\left\{\begin{array}{l}
v_{t}^{i}(x, t)=\frac{v_{x x}^{i}(x, t)}{\left|\gamma_{x}^{i}(x, t)\right|^{2}}-2 \frac{\left\langle v_{x}^{i}(x, t) \mid \gamma_{x}^{i}(x, t)\right\rangle \gamma_{x x}^{i}(x, t)}{\left|\gamma_{x}^{i}(x, t)\right|^{4}} \\
v^{i}(0, t)=v^{j}(0, t) \\
\sum_{i=1}^{3} \frac{v_{x}^{i}(0, t)}{\left|\gamma_{x}^{i}(0, t)\right|}-\frac{\left\langle v_{x}^{i}(0, t) \mid \gamma_{x}^{i}(0, t)\right\rangle \gamma_{x}^{i}(0, t)}{\left|\gamma_{x}^{i}(0, t)\right|^{3}}=0 \\
v^{i}(1, t)=0 \\
v^{i}(x, 0)=\frac{\sigma_{x x}^{i}(x)}{\left|\sigma_{x}^{i}(x)\right|^{2}}
\end{array}\right.
$$

for every $i, j \in\{1,2,3\}$.

This system can be rewritten as

$$
\left\{\begin{array}{l}
v_{t}^{i}(x, t)=v_{x x}^{i}(x, t) f^{i}(x, t)+\left\langle v_{x}^{i}(x, t) \mid g^{i}(x, t)\right\rangle \\
v^{i}(0, t)=v^{j}(0, t) \\
\sum_{i=1}^{3} v_{x}^{i}(0, t) p^{i}(t)+\left\langle v_{x}^{i}(0, t) \mid q^{i}(t)\right\rangle r^{i}(t)=0 \\
v^{i}(1, t)=0 \\
v^{i}(x, 0)=h^{i}(x)
\end{array}\right.
$$

with coefficients

$$
f^{i}, g^{i} \in C^{2 n-2+2 \alpha, n-1+\alpha}\left([0,1] \times\left[0, T_{n}\right]\right), p^{i}, q^{i}, r^{i} \in C^{2 n-1+\alpha, n-1 / 2+\alpha}\left(\left[0, T_{n}\right]\right)
$$

and $h^{i} \in C^{2 n+2 \alpha}([0,1])$, since the initial triod is smooth.

By Solonnikov [35] results, $v^{i}=\gamma_{t}^{i}$ belongs to $C^{2 n+2 \alpha, n+\alpha}\left([0,1] \times\left[0, T_{n}\right]\right)$ and since $\gamma_{x x}^{i}=\gamma_{t}^{i}\left|\gamma_{x}^{i}\right|^{2}$ with $\left|\gamma_{x}^{i}\right|^{2} \in C^{2 n-1+2 \alpha, n-1 / 2+\alpha}\left([0,1] \times\left[0, T_{n}\right]\right)$, we get also $\gamma_{x x}^{i} \in C^{2 n-1+2 \alpha, n-1 / 2+\alpha}\left([0,1] \times\left[0, T_{n}\right]\right)$.

Following [33], we can then conclude that $\gamma^{i} \in C^{2 n+1+2 \alpha, n+1 / 2+\alpha}([0,1] \times$ $\left.\left[0, T_{n}\right]\right)$.

Iterating this argument, we see that $\gamma^{i} \in C^{\infty}\left([0,1] \times\left[0, T_{n}\right]\right)$, moreover, since for every $n \in \mathbb{N}$ the solution obtained via the method of Bronsard and Reitich is unique, it must coincide with $\gamma^{i}$ and we can choose all the $T_{n}$ to be 
the same positive value $T$. Finally, by the same reason, all the compatibility conditions are satisfied at every time, that is, the evolving triods are smooth.

The facts that these triods actually stay in the convex set $\Omega$ and that they do not develop self-intersections during the flow will follow by the results of Section 4 (Proposition 4.1 and Theorem 4.6).

Proposition 3.2. Any solution of Problem (2.1) is a smooth Brakke flow with equality.

Moreover, for every curve $\gamma^{i}(\cdot, t)$ and for every time $t \in[0, T)$ we have

$$
\frac{d L^{i}(t)}{d t}=-\lambda^{i}(0, t)-\int_{\gamma^{i}(\cdot, t)} k^{2} d s
$$

and

$$
\frac{d L(t)}{d t}=-\int_{\mathbb{T}_{t}} k^{2} d s .
$$

Hence, the total length $L(t)$ is decreasing in time and uniformly bounded by the length of the initial triod $\mathbb{T}_{0}$.

Proof. The geometrical smoothness of the flow is clear.

The time derivative of the measure $d s$ on the curve $\gamma^{i}$ is given by $\left(\lambda_{s}^{i}-\right.$ $\left.\left(k^{i}\right)^{2}\right) d s$, considering a smooth function with compact support $\varphi: \Omega \times[0, T) \rightarrow \mathbb{R}$, we compute

$$
\begin{aligned}
\frac{d}{d t} \int_{\gamma^{i}(\cdot, t)} \varphi\left(\gamma^{i}, t\right) d s= & \int_{\gamma^{i}(\cdot, t)} \varphi\left(\gamma^{i}, t\right)\left(\lambda_{s}^{i}-\left(k^{i}\right)^{2}\right) d s+\int_{\gamma^{i}(\cdot, t)}\left\langle\nabla \varphi\left(\gamma^{i}, t\right) \mid \underline{v}^{i}\right\rangle d s \\
& +\int_{\gamma^{i}(\cdot, t)} \varphi_{t}\left(\gamma^{i}, t\right) d s \\
= & \int_{\gamma^{i}(\cdot, t)} \partial_{s}\left(\lambda^{i} \varphi\left(\gamma^{i}, t\right)\right)-\lambda^{i}\left\langle\nabla \varphi\left(\gamma^{i}, t\right) \mid \tau^{i}\right\rangle-\varphi\left(\gamma^{i}, t\right)\left(k^{i}\right)^{2} d s \\
& +\int_{\gamma^{i}(\cdot, t)}\left\langle\nabla \varphi\left(\gamma^{i}, t\right) \mid \underline{v}^{i}\right\rangle d s+\int_{\gamma^{i}(\cdot, t)} \varphi_{t}\left(\gamma^{i}, t\right) d s \\
= & \int_{\gamma^{i}(\cdot, t)} \partial_{s}\left(\lambda^{i} \varphi\left(\gamma^{i}, t\right)\right)-\varphi\left(\gamma^{i}, t\right)\left(k^{i}\right)^{2} d s \\
& +\int_{\gamma^{i}(\cdot, t)}\left\langle\nabla \varphi\left(\gamma^{i}, t\right) \mid \underline{k}^{i}\right\rangle d s+\int_{\gamma^{i}(\cdot, t)} \varphi_{t}\left(\gamma^{i}, t\right) d s \\
= & -\int_{\gamma^{i}(\cdot, t)} \varphi\left(\gamma^{i}, t\right)\left(k^{i}\right)^{2} d s+\int_{\gamma^{i}(\cdot, t)}\left\langle\nabla \varphi\left(\gamma^{i}, t\right) \mid \underline{k}^{i}\right\rangle d s \\
& +\int_{\gamma^{i}(\cdot, t)} \varphi_{t}\left(\gamma^{i}, t\right) d s+\lambda^{i}(1, t) \varphi\left(P^{i}, t\right)-\lambda^{i}(0, t) \varphi(O(t), t) .
\end{aligned}
$$

Since $\lambda^{i}(1, t)$ is zero, being zero the velocity $v$ at the end points $P^{i}$, and since the sum of $\lambda^{i}$ is zero at the 3-point, adding these three equalities for $i \in\{1,2,3\}$ we obtain formula (2.11).

The formulas for the lengths are given by the same computation with $\varphi \equiv 1$. 
Seeing the initial triod $\mathbb{T}_{0}$ simply as a subset of $\mathbb{R}^{2}$, it can admit more than a single parametrization of its curves making it a smooth triod, so there are various flows arising by this theorem, associated to different parametrizations. The following proposition shows that they must coincide geometrically.

Proposition 3.3. If $\mathbb{T}_{0}^{1}=\cup_{i=1}^{3} \sigma_{0}^{i}$ and $\mathbb{T}_{0}^{2}=\cup_{i=1}^{3} \xi_{0}^{i}$ are two smooth triods which coincide as subset of $\mathbb{R}^{2}$ and $\mathbb{T}_{t}^{1}, \mathbb{T}_{t}^{2}$ are the relative flows given by Theorem 3.1 on a common time interval $[0, T)$, then at every time $t$ the triods $\mathbb{T}_{t}^{1}$ and $\mathbb{T}_{t}^{2}$ coincide as sets.

Proof. Let $\gamma^{i}(x, t)$ and $\eta^{i}(x, t)$ be the two smooth flows associated to $\sigma^{i}=\gamma^{i}(\cdot, 0)$ and $\xi^{i}=\eta^{i}(\cdot, 0)$, which parametrize the same triod, seen as a subset of $\mathbb{R}^{2}$.

We fix an index $i \in\{1,2,3\}$. Since $\sigma^{i}$ and $\xi^{i}$ are smooth regular parametrization of the same curve of the initial triod, the map $\varphi^{i}=\left(\sigma^{i}\right)^{-1} \circ \xi^{i}$ : $[0,1] \rightarrow[0,1]$ is an orientation preserving, smooth diffeomorphisms of the unit interval with itself, hence $\varphi^{i}(0)=0$ and $\varphi^{i}(1)=1$. Moreover, by the compatibility conditions we have $\sigma_{x x}^{i}(1)=\xi_{x x}^{i}(1)=\left(\sigma^{i} \circ \varphi^{i}\right)_{x x}(1)=0$, hence

$$
0=\sigma_{x x}^{i}\left(\varphi^{i}(1)\right)\left|\varphi_{x}^{i}(1)\right|^{2}+\sigma_{x}^{i}\left(\varphi^{i}(1)\right) \varphi_{x x}^{i}(1)=\sigma_{x}^{i}\left(\varphi^{i}(1)\right) \varphi_{x x}^{i}(1)=\sigma_{x}^{i}(1) \varphi_{x x}^{i}
$$

which implies that $\varphi_{x x}^{i}(1)=0$. At the 3-point, we have

$$
\begin{aligned}
\frac{\xi_{x x}^{i}(0)}{\left|\xi_{x}^{i}(0)\right|^{2}}-\frac{\sigma_{x x}^{i}(0)}{\left|\sigma_{x}^{i}(0)\right|^{2}} & =\frac{\sigma_{x x}^{i}\left(\varphi^{i}(0)\right)\left|\varphi_{x}^{i}(0)\right|^{2}+\sigma_{x}^{i}\left(\varphi^{i}(0)\right) \varphi_{x x}^{i}(0)}{\left|\sigma_{x}^{i}\left(\varphi^{i}(0)\right) \varphi_{x}^{i}(0)\right|^{2}}-\frac{\sigma_{x x}^{i}(0)}{\left|\sigma_{x}^{i}(0)\right|^{2}} \\
& =\frac{\sigma_{x x}^{i}(0)\left|\varphi_{x}^{i}(0)\right|^{2}+\sigma_{x}^{i}(0) \varphi_{x x}^{i}(0)-\sigma_{x x}^{i}(0)\left|\varphi_{x}^{i}(0)\right|^{2}}{\left|\sigma_{x}^{i}(0) \varphi_{x}^{i}(0)\right|^{2}} \\
& =\frac{\sigma_{x}^{i}(0) \varphi_{x x}^{i}(0)}{\left|\sigma_{x}^{i}(0) \varphi_{x}^{i}(0)\right|^{2}}
\end{aligned}
$$

hence, $\frac{\sigma_{x}^{i}(0) \varphi_{x x}^{i}(0)}{\left|\sigma_{x}^{i}(0) \varphi_{x}^{i}(0)\right|^{2}}=\frac{\sigma_{x}^{j}(0) \varphi_{x x}^{j}(0)}{\left|\sigma_{x}^{j}(0) \varphi_{x}^{j}(0)\right|^{2}}$ for every pair $i, j \in\{1,2,3\}$. This means that $\varphi_{x x}^{i}(0)=0$ since the tangents to the curves $\sigma^{i}$ and $\sigma^{j}$ are not parallel.

Now we look for three smooth functions $\psi^{i}:[0,1] \times[0, T) \rightarrow[0,1]$ satisfying the following parabolic system

$$
\left\{\begin{array}{l}
\psi_{t}^{i}(x, t)=\frac{\psi_{x x}^{i}(x, t)}{\left|\gamma_{x}^{i}\left(\psi^{i}(x, t), t\right)\right|^{2}\left|\psi_{x}^{i}(x, t)\right|^{2}} \\
\psi^{i}(0, t)=0 \\
\psi^{i}(1, t)=1 \\
\psi^{i}(x, 0)=\varphi^{i}(x)
\end{array}\right.
$$

for every $(x, t) \in[0,1] \times[0, T)$ and every index $i \in\{1,2,3\}$.

We see that the compatibility conditions of order 2 are satisfied by the initial data, indeed, here these reduce only to $\varphi_{x x}^{i}(0)=\varphi_{x x}^{i}(1)=0$. 
By standard methods (now the problem is scalar, see [32], [35]), being $\varphi^{i}$ smooth and regular $\left(\varphi_{x}^{i}(x) \neq 0\right.$ for every $x \in[0,1]$ since it is a diffeomorphisms), the functions $\gamma_{x}^{i}$ bounded from above and away from zero, and holding the compatibility conditions of order 0,1 and 2 , this quasilinear problem has a solution on some maximal time interval $\left[0, T^{\prime}\right)$, with $T^{\prime} \leq T$, belonging to $C^{2+2 \alpha, 1+\alpha}\left([0,1] \times\left[0, T^{\prime \prime}\right]\right)$ (for some $\left.\alpha \in(0,1 / 2)\right)$ for every time $T^{\prime \prime} \in\left(0, T^{\prime}\right)$.

It is now straightforward to see that the functions $\theta^{i}(x, t)=\gamma^{i}\left(\psi^{i}(x, t), t\right)$ coincide with $\eta^{i}(x, t)$ at time $t=0$ (indeed, $\xi^{i}=\sigma^{i} \circ \varphi^{i}$ ) and

$$
\begin{aligned}
\frac{\partial \theta^{i}(x, t)}{\partial t} & =\frac{\partial \gamma^{i}\left(\psi^{i}(x, t), t\right)}{\partial t} \\
& =\gamma_{t}^{i}\left(\psi^{i}(x, t), t\right)+\gamma_{x}^{i}\left(\psi^{i}(x, t), t\right) \psi_{t}^{i}(x, t) \\
& =\frac{\gamma_{x x}^{i}\left(\psi^{i}(x, t), t\right)}{\left|\gamma_{x}^{i}\left(\psi^{i}(x, t), t\right)\right|^{2}}+\gamma_{x}^{i}\left(\psi^{i}(x, t), t\right) \frac{\psi_{x x}^{i}(x, t)}{\left|\gamma_{x}^{i}\left(\psi^{i}(x, t), t\right)\right|^{2}\left|\psi_{x}^{i}(x, t)\right|^{2}} \\
& =\frac{\gamma_{x x}^{i}\left(\psi^{i}(x, t), t\right)\left|\psi_{x}^{i}(x, t)\right|^{2}+\gamma_{x}^{i}\left(\psi^{i}(x, t), t\right) \psi_{x x}^{i}(x, t)}{\left|\gamma_{x}^{i}(\psi(x, t), t)\right|^{2}\left|\psi_{x}^{i}(x, t)\right|^{2}} \\
& =\frac{\left(\gamma^{i}\left(\psi^{i}(x, t), t\right)\right)_{x x}}{\left|\left(\gamma^{i}\left(\psi^{i}(x, t), t\right)\right)_{x}\right|^{2}}=\frac{\theta_{x x}^{i}(x, t)}{\left|\theta_{x}^{i}(x, t)\right|^{2}}
\end{aligned}
$$

Then, since such functions $\theta^{i}$ and $\eta^{i}$ satisfy both system (3.1) and they take the same initial data at time $t=0$, they must coincide on $\left[0, T^{\prime}\right)$, by the uniqueness of the solution in $C^{2+2 \alpha, 1+\alpha}$ proved by Bronsard and Reitich in [11].

At the maximal time $T^{\prime}$ it has to happen that $\psi_{x}^{i}$ is no more bounded away from zero or the $C^{2+2 \alpha}$ norm of $\psi^{i}(\cdot, t)$ is not bounded from above, but a simple computation shows that then the same holds for $\theta^{i}$ hence also for $\eta^{i}$, which is smooth and regular on $[0, T)$.

This clearly implies that $T^{\prime}=T$ and that the triods $\mathbb{T}_{t}^{1}$ and $\mathbb{T}_{t}^{2}$ are the same subset of $\mathbb{R}^{2}$ for every time $t$ in the interval $[0, T)$.

REMARK 3.4. This proposition clearly sets positively the question about the geometric uniqueness of the flow of a smooth triod. Actually, we do not know if, at least in this special initial case, uniqueness holds also in the class of smooth Brakke flows with equality.

Clearly, if a triod is geometrically smooth but not smooth, we can reparametrize it and apply Theorem 3.1 in order to get a smooth flow (which is a smooth Brakke flow with equality).

Now in order to improve these results and to study the global existence and regularity of the evolution in the next sections, we work out a priori estimates for $k^{i}, \lambda^{i}$ and their derivatives.

REMARK 3.5. Sometimes we will consider time depending functions, defined as the maximum of a geometric quantity over the triods, in order to get estimates by means of ODE's and maximum principle arguments. Even if the evolution 
is smooth, such functions will be typically only Lipschitz, hence they can fail to be differentiable at some times, so there will be a little misuse of notation in writing a derivative that possibly does not exist at every time. However, the arguments used, which are pointwise and apparently affected by the lack of differentiability, still work also in this situation, as explained in details by Hamilton in [21, Sections 3 and 4].

We fix some non standard notation for the computations in the sequel.

We denote with $\mathfrak{p}_{\sigma}\left(\partial_{s}^{j} \lambda, \partial_{s}^{h} k\right)$ a polynomial in $\lambda, \ldots, \partial_{s}^{j} \lambda$ and $k, \ldots, \partial_{s}^{h} k$ with constant coefficients, such that every monomial it contains is of the form

$$
C \prod_{l=0}^{j}\left(\partial_{s}^{l} \lambda\right)^{\alpha_{l}} \cdot \prod_{l=0}^{h}\left(\partial_{s}^{l} k\right)^{\beta_{l}} \quad \text { with } \sum_{l=0}^{j}(l+1) \alpha_{l}+\sum_{l=0}^{h}(l+1) \beta_{l}=\sigma,
$$

we will call $\sigma$ the geometric order of $\mathfrak{p}_{\sigma}$.

Moreover, if one of the two arguments of $\mathfrak{p}_{\sigma}$ does not appear, it means that the polynomial does not contain it, for instance, $\mathfrak{p}_{\sigma}\left(\partial_{s}^{h} k\right)$ does not contain neither $\lambda$ nor its derivatives.

We denote with $\mathfrak{q}_{\sigma}\left(\partial_{t}^{j} \lambda, \partial_{s}^{h} k\right)$ a polynomial as before in $\lambda, \ldots, \partial_{t}^{j} \lambda$ and $k, \ldots, \partial_{s}^{h} k$ such that all its monomials are of the form

$$
C \prod_{l=0}^{j}\left(\partial_{t}^{l} \lambda\right)^{\alpha_{l}} \cdot \prod_{l=0}^{h}\left(\partial_{s}^{l} k\right)^{\beta_{l}} \quad \text { with } \sum_{l=0}^{j}(2 l+1) \alpha_{l}+\sum_{l=0}^{h}(l+1) \beta_{l}=\sigma .
$$

Finally, when we will write $\mathfrak{p}_{\sigma}\left(\left|\partial_{s}^{j} \lambda\right|,\left|\partial_{s}^{h} k\right|\right)\left(\right.$ or $\left.\mathfrak{q}_{\sigma}\left(\left|\partial_{t}^{j} \lambda\right|,\left|\partial_{s}^{h} k\right|\right)\right)$ we will mean a finite sum of terms like

$$
C \prod_{l=0}^{j}\left|\partial_{s}^{l} \lambda\right|^{\alpha_{l}} \cdot \prod_{l=0}^{h}\left|\partial_{s}^{l} k\right|^{\beta_{l}} \quad \text { with } \sum_{l=0}^{j}(l+1) \alpha_{l}+\sum_{l=0}^{h}(l+1) \beta_{l}=\sigma,
$$

where $C$ is a positive constant and the exponents $\alpha_{l}, \beta_{l}$ are non negative real values (analogously for $\mathfrak{q}_{\sigma}$ ).

Clearly we have $\mathfrak{p}_{\sigma}\left(\partial_{s}^{j} \lambda, \partial_{s}^{h} k\right) \leq \mathfrak{p}_{\sigma}\left(\left|\partial_{s}^{j} \lambda\right|,\left|\partial_{s}^{h} k\right|\right)$.

REMARK 3.6. We advise the reader that in the following computations these polynomials can vary from one line to another, by addition of similar terms, what has to be kept in mind is that the coefficients and the number of monomials they contains are independent of $k, \lambda$ and their derivatives, since they arise by the algorithmic construction of the polynomials.

We will often denote with $C$ a generic constant which also can vary from one passage to another.

We will make extensive use of Young inequality in the following form $a b \leq \varepsilon a^{p}+C(\varepsilon, p, q) b^{q} \quad$ for $a, b, \varepsilon<0, p, q \in(1, \infty)$ and $1 / p+1 / q=1$. 


\section{LEMma 3.7. The following formulas hold}

$$
\begin{array}{ll}
\partial_{t} \partial_{s}^{j} k=\partial_{s}^{j+2} k+\lambda \partial_{s}^{j+1} k+\mathfrak{p}_{j+3}\left(\partial_{s}^{j} k\right) & \text { for every } j \in \mathbb{N}, \\
\partial_{s}^{j} k=\partial_{t}^{j / 2} k+\mathfrak{q}_{j+1}\left(\partial_{t}^{j / 2-1} \lambda, \partial_{s}^{j-1} k\right) & \text { if } j \geq 2 \text { is even, } \\
\partial_{s}^{j} k=\partial_{t}^{(j-1) / 2} k_{s}+\mathfrak{q}_{j+1}\left(\partial_{t}^{(j-3) / 2} \lambda, \partial_{s}^{j-1} k\right) & \text { if } j \geq 1 \text { is odd. }
\end{array}
$$

Proof. The case $j=0$ of the first formula is equation (2.6). Suppose that the formula holds for $(j-1)$, using the commutation rule (23) we have

$$
\begin{aligned}
\partial_{t} \partial_{s}^{j} k & =\partial_{s} \partial_{t} \partial_{s}^{j-1} k+\left(k^{2}-\lambda_{s}\right) \partial_{s}^{j} k \\
& =\partial_{s}\left[\partial_{s}^{j+1} k+\lambda \partial_{s}^{j} k+\mathfrak{p}_{j+2}\left(\partial_{s}^{j-1} k\right)\right]-\lambda_{s} \partial_{s}^{j} k+\mathfrak{p}_{j+3}\left(\partial_{s}^{j} k\right) \\
& =\partial_{s}^{j+2} k+\lambda_{s} \partial_{s}^{j} k+\lambda \partial_{s}^{j+1} k+\mathfrak{p}_{j+3}\left(\partial_{s}^{j} k\right)-\lambda_{s} \partial_{s}^{j} k+\mathfrak{p}_{j+3}\left(\partial_{s}^{j} k\right) \\
& =\partial_{s}^{j+2} k+\lambda \partial_{s}^{j+1} k+\mathfrak{p}_{j+3}\left(\partial_{s}^{j} k\right)
\end{aligned}
$$

which gives the inductive step.

The second formula also follows by induction. The case $j=2$ is again equation (2.6). If the case $(j-2)$ holds, then by the first formula,

$$
\begin{aligned}
\partial_{s}^{j} k & =\partial_{t} \partial_{s}^{j-2} k-\lambda \partial_{s}^{j-1} k+\mathfrak{p}_{j+1}\left(\partial_{s}^{j-2} k\right)=\partial_{t}\left[\partial_{t}^{j / 2-1} k+\mathfrak{q}_{j-1}\left(\partial_{t}^{j / 2-2} \lambda, \partial_{s}^{j-3} k\right)\right] \\
& =\partial_{t}^{j / 2} k+\partial_{t} \mathfrak{q}_{j-1}\left(\partial_{t}^{j / 2-2} \lambda, \partial_{s}^{j-3} k\right) .
\end{aligned}
$$

Now, when we differentiate in $t$ the term $\mathfrak{q}_{j-1}\left(\partial_{t}^{j / 2-2} \lambda, \partial_{s}^{j-3} k\right)$ we will get a polynomial in $\lambda, \ldots, \partial_{t}^{j / 2-1} \lambda, k, \ldots \partial_{s}^{j-1} k$ and time derivatives of space derivatives of $k$. Using the first formula we can express these latter as polynomials in $\lambda$ and space derivatives of $k$, up to the order $\partial_{s}^{j-1} k$. Moreover, it is easy to check that the resulting polynomial is of the form $\mathfrak{q}_{j+1}\left(\partial_{t}^{j / 2-1} \lambda, \partial_{s}^{j-1} k\right)$, hence the formula for $j$ is proved.

The odd case is analogous.

\section{LEMma 3.8. The following formulas hold}

$$
\begin{array}{ll}
\partial_{t} \partial_{s}^{j} \lambda=\partial_{s}^{j+2} \lambda-\lambda \partial_{s}^{j+1} \lambda-2 k \partial_{s}^{j+1} k+\mathfrak{p}_{j+3}\left(\partial_{s}^{j} \lambda, \partial_{s}^{j} k\right) & \text { for every } j \in \mathbb{N}, \\
\partial_{s}^{j} \lambda=\partial_{t}^{j / 2} \lambda+\mathfrak{p}_{j+1}\left(\partial_{s}^{j-1} \lambda, \partial_{s}^{j-1} k\right) & \text { if } j \geq 2 \text { is even, } \\
\partial_{s}^{j} \lambda=\partial_{t}^{(j-1) / 2} \lambda_{s}+\mathfrak{p}_{j+1}\left(\partial_{s}^{j-1} \lambda, \partial_{s}^{j-1} k\right) & \text { if } j \geq 1 \text { is odd. }
\end{array}
$$

PROOF. The case $j=0$ of the first formula is equation (2.7), then the proof follows as for $k$ in the previous lemma. 
REMARK 3.9. We state the following calculus rules which will be used extensively in the sequel,

$$
\begin{aligned}
& \mathfrak{p}_{\alpha}\left(\partial_{s}^{j} \lambda, \partial_{s}^{h} k\right) \cdot \mathfrak{p}_{\beta}\left(\partial_{s}^{l} \lambda, \partial_{s}^{m} k\right)=\mathfrak{p}_{\alpha+\beta}\left(\partial_{s}^{\max \{j, l\}} \lambda, \partial_{s}^{\max \{h, m\}} k\right), \\
& \mathfrak{q}_{\alpha}\left(\partial_{t}^{j} \lambda, \partial_{s}^{h} k\right) \cdot \mathfrak{q}_{\beta}\left(\partial_{t}^{l} \lambda, \partial_{s}^{m} k\right)=\mathfrak{q}_{\alpha+\beta}\left(\partial_{t}^{\max \{j, l\}} \lambda, \partial_{s}^{\max \{h, m\}} k\right) .
\end{aligned}
$$

Since the time derivatives of $k$ and $\lambda$ and their space derivatives can be expressed in terms of these latter, by means of Lemmas 3.7 and 3.8, we have

$$
\begin{array}{ll}
\partial_{s}^{l} \mathfrak{p}_{\alpha}\left(\partial_{s}^{j} \lambda, \partial_{s}^{h} k\right)=\mathfrak{p}_{\alpha+l}\left(\partial_{s}^{j+l} \lambda, \partial_{s}^{h+l} k\right), & \partial_{t}^{l} \mathfrak{p}_{\alpha}\left(\partial_{s}^{j} \lambda, \partial_{s}^{h} k\right)=\mathfrak{p}_{\alpha+2 l}\left(\partial_{s}^{j+2 l} \lambda, \partial_{s}^{h+2 l} k\right) \\
\partial_{t}^{l} \mathfrak{q}_{\alpha}\left(\partial_{t}^{j} \lambda, \partial_{s}^{h} k\right)=\mathfrak{q}_{\alpha+2 l}\left(\partial_{t}^{j+l} \lambda, \partial_{s}^{h+2 l} k\right), & \mathfrak{q}_{\alpha}\left(\partial_{t}^{j} \lambda, \partial_{s}^{h} k\right)=\mathfrak{p}_{\alpha}\left(\partial_{s}^{2 j} \lambda, \partial_{s}^{\max \{h, 2 j-1\}} k\right) .
\end{array}
$$

Moreover, by relations (2.9), at the 3-point $\partial_{t}^{j} \lambda^{i}=\left(\mathrm{S} \partial_{t}^{j} \mathrm{~K}\right)^{i}$, that is, the time derivatives of $\lambda^{i}$ are expressible as time derivatives of the functions $k^{i}$. Then, by using repeatedly such relation and the first formula of Lemma 3.7, we can express these latter as space derivatives of $k^{i}$. Hence, we have the relation

$$
\left.\sum_{i=1}^{3} \mathfrak{q}_{\sigma}\left(\partial_{t}^{j} \lambda^{i}, \partial_{s}^{h} k^{i}\right)\right|_{\text {at the 3-point }}=\left.\mathfrak{p}_{\sigma}\left(\partial_{s}^{\max \{2 j, h\}} \mathrm{K}\right)\right|_{\text {at the 3-point }}
$$

with the meaning that this last polynomial contains also product of derivatives of different $k^{i}$, s, because of the action of the linear operator $\mathrm{S}: \mathbb{R}^{3} \rightarrow \mathbb{R}^{3}$.

We will often make use of this identity in the computations in the sequel in the following form,

$$
\left.\sum_{i=1}^{3} \mathfrak{q}_{\sigma}\left(\partial_{t}^{j} \lambda^{i}, \partial_{s}^{h} k^{i}\right)\right|_{\text {at the 3-point }} \leq\left\|\mathfrak{p}_{\sigma}\left(\left|\partial_{s}^{\max \{2 j, h\}} k\right|\right)\right\|_{L^{\infty}}
$$

Before proceeding we prove also a relation holding at the end points.

LeMma 3.10. At the three end points $P^{i}$ there holds $\partial_{s}^{j} k^{i}=\partial_{s}^{j} \lambda^{i}=0$, for every even $j \in \mathbb{N}$.

Proof. The first case $j=0$ simply follows from the fact that the velocity $\underline{v}=\lambda \tau+k v$ is always zero at the three fixed end points $P^{i}$.

We argue by induction, we suppose that for every even natural $l \leq j-2$ we have $\partial_{s}^{l} k^{i}=\partial_{s}^{l} \lambda^{i}=0$, then, by using the first equation in Lemma 3.7 , we get

$$
\partial_{s}^{j} k^{i}=\partial_{t} \partial_{s}^{j-2} k^{i}-\lambda^{i} \partial_{s}^{j-1} k^{i}-\mathfrak{p}_{j+1}\left(\partial_{s}^{j-2} k^{i}\right)
$$

at the points $P^{i}$.

We already know that $\lambda^{i}=0$ and by the inductive hypothesis $\partial_{s}^{j-2} k^{i}=0$, thus $\partial_{t} \partial_{s}^{j-2} k^{i}=0$. Since $\mathfrak{p}_{j+1}\left(\partial_{s}^{j-2} k^{i}\right)$ is a sum of terms like $C \prod_{l=0}^{j-2}\left(\partial_{s}^{l} k^{i}\right)^{\alpha_{l}}$ with $\sum_{l=0}^{j-2}(l+1) \alpha_{l}=j+1$ which is odd, at least one of the terms of this 
sum has to be odd, hence at least for one index $l$, the product $(l+1) \alpha_{l}$ is odd. It follows that at least for one even $l$ the exponent $\alpha_{l}$ is nonzero. Hence, at least one even derivatives is present in every monomial of $\mathfrak{p}_{j+1}\left(\partial_{s}^{j-2} k^{i}\right)$, which contains only derivatives up to the order $(j-2)$.

Again, by the inductive hypothesis we then conclude that at the end points $\partial_{s}^{j} k^{i}=0$.

We can deal with $\lambda^{i}$ similarly, by means of the first equation of Lemma 3.8.

Taking into account that the time derivative of the measure $d s$ is given by $\left(\lambda_{s}-k^{2}\right) d s$ and using the first relation of Lemma 3.7, we compute for $j \in \mathbb{N}$

$$
\begin{aligned}
\frac{d}{d t} \int_{\mathbb{T}_{t}}\left|\partial_{s}^{j} k\right|^{2} d s= & 2 \int_{\mathbb{T}_{t}} \partial_{s}^{j} k \partial_{t} \partial_{s}^{j} k d s+\int_{\mathbb{T}_{t}}\left|\partial_{s}^{j} k\right|^{2}\left(\lambda_{s}-k^{2}\right) d s \\
= & 2 \int_{\mathbb{T}_{t}} \partial_{s}^{j} k \partial_{s}^{j+2} k+\lambda \partial_{s}^{j+1} k \partial_{s}^{j} k+\mathfrak{p}_{j+3}\left(\partial_{s}^{j} k\right) \partial_{s}^{j} k d s \\
& +\int_{\mathbb{T}_{t}}\left|\partial_{s}^{j} k\right|^{2}\left(\lambda_{s}-k^{2}\right) d s \\
= & -2 \int_{\mathbb{T}_{t}}\left|\partial_{s}^{j+1} k\right|^{2} d s+\int_{\mathbb{T}_{t}} \partial_{s}\left(\lambda\left|\partial_{s}^{j} k\right|^{2}\right) d s \\
& +\int_{\mathbb{T}_{t}} \mathfrak{p}_{2 j+4}\left(\partial_{s}^{j} k\right) d s-\left.2 \sum_{i=1}^{3} \partial_{s}^{j} k^{i} \partial_{s}^{j+1} k^{i}\right|_{\text {at the 3-point }} \\
& +\left.2 \sum_{i=1}^{3} \partial_{s}^{j} k^{i} \partial_{s}^{j+1} k^{i}\right|_{\text {at the point }} P^{i} \\
= & -2 \int_{\mathbb{T}_{t}}\left|\partial_{s}^{j+1} k\right|^{2} d s+\int_{\mathbb{T}_{t}} \mathfrak{p}_{2 j+4}\left(\partial_{s}^{j} k\right) d s \\
& -\sum_{i=1}^{3} 2 \partial_{s}^{j} k^{i} \partial_{s}^{j+1} k^{i}+\left.\lambda^{i}\left|\partial_{s}^{j} k^{i}\right|^{2}\right|_{\text {at the 3-point }}
\end{aligned}
$$

where we integrated by parts a couple of times and we eliminated the contributions given by the end points $P^{i}$ by means of Lemma 3.10.

In the very special (and important as we will see) case $j=0$ we get explicitly

$$
\frac{d}{d t} \int_{\mathbb{T}_{t}} k^{2} d s=-2 \int_{\mathbb{T}_{t}}\left|k_{s}\right|^{2} d s+\int_{\mathbb{T}_{t}} k^{4} d s-\sum_{i=1}^{3} 2 k^{i} k_{s}^{i}+\left.\lambda^{i}\left(k^{i}\right)^{2}\right|_{\text {at the 3-point }} .
$$

Then, recalling relation (2.10) with $l, m=0$, we have

$$
\sum_{i=1}^{3} k^{i} k_{s}^{i}+\left.\lambda^{i}\left(k^{i}\right)^{2}\right|_{\text {at the 3-point }}=0,
$$


and substituting in the last term above,

$$
\frac{d}{d t} \int_{\mathbb{T}_{t}} k^{2} d s=-2 \int_{\mathbb{T}_{t}}\left|k_{s}\right|^{2} d s+\int_{\mathbb{T}_{t}} k^{4} d s+\left.\sum_{i=1}^{3} \lambda^{i}\left(k^{i}\right)^{2}\right|_{\text {at the 3-point }}
$$

hence, we lowered the maximum order of the space derivatives of the curvature in the 3-point term, particular now it is lower than the one of the "nice" negative integral. even.

Now we are going to do the same for the general case, when $j \geq 2$ is

By means of formulas (3.3) we have

$$
\begin{aligned}
& 2 \partial_{s}^{j} k \partial_{s}^{j+1} k+\lambda\left|\partial_{s}^{j} k\right|^{2} \\
&= 2\left[\partial_{t}^{j / 2} k+\mathfrak{q}_{j+1}\left(\partial_{t}^{j / 2-1} \lambda, \partial_{s}^{j-1} k\right)\right] \cdot\left[\partial_{t}^{j / 2} k_{s}+\mathfrak{q}_{j+2}\left(\partial_{t}^{j / 2-1} \lambda, \partial_{s}^{j} k\right)\right] \\
&+\mathfrak{q}_{2 j+3}\left(\lambda, \partial_{s}^{j} k\right) \\
&= 2\left[\partial_{t}^{j / 2} k+\mathfrak{q}_{j+1}\left(\partial_{t}^{j / 2-1} \lambda, \partial_{s}^{j-1} k\right)\right] \cdot\left[\partial_{t}^{j / 2}\left(k_{s}+k \lambda\right)+\mathfrak{q}_{j+2}\left(\partial_{t}^{j / 2} \lambda, \partial_{s}^{j} k\right)\right] \\
&+\mathfrak{q}_{2 j+3}\left(\lambda, \partial_{s}^{j} k\right) \\
&= 2 \partial_{t}^{j / 2} k \cdot \partial_{t}^{j / 2}\left(k_{s}+k \lambda\right)+\partial_{t}^{j / 2} k \cdot \mathfrak{q}_{j+2}\left(\partial_{t}^{j / 2} \lambda, \partial_{s}^{j} k\right) \\
&+\mathfrak{q}_{j+1}\left(\partial_{t}^{j / 2-1} \lambda, \partial_{s}^{j-1} k\right) \cdot \partial_{t}^{j / 2}\left(k_{s}+k \lambda\right) \\
&+\mathfrak{q}_{j+1}\left(\partial_{t}^{j / 2-1} \lambda, \partial_{s}^{j-1} k\right) \cdot \mathfrak{q}_{j+2}\left(\partial_{t}^{j / 2} \lambda, \partial_{s}^{j} k\right)+\mathfrak{q}_{2 j+3}\left(\lambda, \partial_{s}^{j} k\right) \\
&= 2 \partial_{t}^{j / 2} k \cdot \partial_{t}^{j / 2}\left(k_{s}+k \lambda\right)+\mathfrak{q}_{j+1}\left(\partial_{t}^{j / 2-1} \lambda, \partial_{s}^{j-1} k\right) \cdot \partial_{t}^{j / 2} k_{s} \\
&+\mathfrak{q}_{2 j+3}\left(\partial_{t}^{j / 2} \lambda, \partial_{s}^{j} k\right) .
\end{aligned}
$$

We now examine the term $\mathfrak{q}_{j+1}\left(\partial_{t}^{j / 2-1} \lambda, \partial_{s}^{j-1} k\right) \cdot \partial_{t}^{j / 2} k_{s}$, which contains $(j+1)$-th space derivatives of $k$ (after expansion of the $j / 2$-th time derivative of $k_{s}$ ).

By using the third relation of Lemma 3.7, it can be written as

$$
\begin{aligned}
\mathfrak{q}_{j+1}\left(\partial_{t}^{j / 2-1} \lambda, \partial_{s}^{j-1} k\right) & \cdot \partial_{t}\left[\partial_{s}^{j-1} k+\mathfrak{q}_{j}\left(\partial_{t}^{j / 2-2} \lambda, \partial_{s}^{j-2} k\right)\right] \\
& =\mathfrak{q}_{j+1}\left(\partial_{t}^{j / 2-1} \lambda, \partial_{s}^{j-1} k\right) \cdot\left[\partial_{t} \partial_{s}^{j-1} k+\mathfrak{q}_{j+2}\left(\partial_{t}^{j / 2-1} \lambda, \partial_{s}^{j} k\right)\right] \\
& =\mathfrak{q}_{j+1}\left(\partial_{t}^{j / 2-1} \lambda, \partial_{s}^{j-1} k\right) \cdot \partial_{t} \partial_{s}^{j-1} k+\mathfrak{q}_{2 j+3}\left(\partial_{t}^{j / 2-1} \lambda, \partial_{s}^{j} k\right),
\end{aligned}
$$

moreover, if we look at the polynomial $\mathfrak{q}_{j+1}\left(\partial_{t}^{j / 2-1} \lambda, \partial_{s}^{j-1} k\right)$, we can see that among its monomials, only those of the form $A \lambda \partial_{s}^{j-1} k$ or $B k \partial_{s}^{j-1} k$ can contain 
the derivative $\partial_{s}^{j-1} k$ (because of the geometric order of $\mathfrak{p}_{2 k+1}$ ). Hence,

$$
\begin{aligned}
& \mathfrak{q}_{j+1}\left(\partial_{t}^{j / 2-1} \lambda, \partial_{s}^{j-1} k\right) \cdot \partial_{t}^{j / 2} k_{s} \\
&= {\left[\mathfrak{q}_{j+1}\left(\partial_{t}^{j / 2-1} \lambda, \partial_{s}^{j-2} k\right)+A \lambda \partial_{s}^{j-1} k+B k \partial_{s}^{j-1} k\right] \cdot \partial_{t} \partial_{s}^{j-1} k } \\
&+\mathfrak{q}_{2 j+3}\left(\partial_{t}^{j / 2-1} \lambda, \partial_{s}^{j} k\right) \\
&=\left(A \lambda \partial_{s}^{j-1} k+B k \partial_{s}^{j-1} k\right) \cdot \partial_{t} \partial_{s}^{j-1} k+\mathfrak{q}_{2 j+3}\left(\partial_{t}^{j / 2-1} \lambda, \partial_{s}^{j} k\right) \\
&+\partial_{t}\left[\mathfrak{q}_{j+1}\left(\partial_{t}^{j / 2-1} \lambda, \partial_{s}^{j-2} k\right) \partial_{s}^{j-1} k\right]-\partial_{t} \mathfrak{q}_{j+1}\left(\partial_{t}^{j / 2-1} \lambda, \partial_{s}^{j-2} k\right) \partial_{s}^{j-1} k \\
&=(A \lambda+B k) \partial_{t}\left(\partial_{s}^{j-1} k / 2\right)^{2}+\partial_{t} \mathfrak{q}_{2 j+1}\left(\partial_{t}^{j / 2-1} \lambda, \partial_{s}^{j-1} k\right)+\mathfrak{q}_{2 j+3}\left(\partial_{t}^{j / 2} \lambda, \partial_{s}^{j} k\right) \\
&= \partial_{t}\left[(A \lambda+B k)\left(\partial_{s}^{j-1} k / 2\right)^{2}\right]-\left(A \partial_{t} \lambda+B \partial_{t} k\right)\left(\partial_{s}^{j-1} k / 2\right)^{2} \\
&+\partial_{t} \mathfrak{q}_{2 j+1}\left(\partial_{t}^{j / 2-1} \lambda, \partial_{s}^{j-1} k\right)+\mathfrak{q}_{2 j+3}\left(\partial_{t}^{j / 2} \lambda, \partial_{s}^{j} k\right) \\
&= \partial_{t} \mathfrak{q}_{2 j+1}\left(\partial_{t}^{j / 2-1} \lambda, \partial_{s}^{j-1} k\right)+\mathfrak{q}_{2 j+3}\left(\partial_{t}^{j / 2} \lambda, \partial_{s}^{j} k\right) .
\end{aligned}
$$

It follows that

$$
\begin{aligned}
& \sum_{i=1}^{3} 2 \partial_{s}^{j} k^{i} \partial_{s}^{j+1} k^{i}+\left.\lambda^{i}\left|\partial_{s}^{j} k^{i}\right|^{2} \lambda\right|_{\text {at the 3-point }} \\
& =\sum_{i=1}^{3} 2 \partial_{t}^{j / 2} k^{i} \cdot \partial_{t}^{j / 2}\left(k_{s}^{i}+k^{i} \lambda^{i}\right) \\
& \quad+\partial_{t} \mathfrak{q}_{2 j+1}\left(\partial_{t}^{j / 2-1} \lambda^{i}, \partial_{s}^{j-1} k^{i}\right)+\left.\mathfrak{q}_{2 j+3}\left(\partial_{t}^{j / 2} \lambda^{i}, \partial_{s}^{j} k^{i}\right)\right|_{\text {at the 3-point }} \\
& \quad=\sum_{i=1}^{3} \partial_{t} \mathfrak{q}_{2 j+1}\left(\partial_{t}^{j / 2-1} \lambda^{i}, \partial_{s}^{j-1} k^{i}\right)+\left.\mathfrak{q}_{2 j+3}\left(\partial_{t}^{j / 2} \lambda^{i}, \partial_{s}^{j} k^{i}\right)\right|_{\text {at the 3-point }}
\end{aligned}
$$

by relations $(2.10)$.

Resuming, if $j \geq 2$ is even, we have

$$
\begin{aligned}
\frac{d}{d t} \int_{\mathbb{T}_{t}}\left|\partial_{s}^{j} k\right|^{2} d s= & -\int_{\mathbb{T}_{t}}\left|\partial_{s}^{j+1} k\right|^{2} d s+\int_{\mathbb{T}_{t}} \mathfrak{p}_{2 j+4}\left(\partial_{s}^{j} k\right) d s \\
& +\sum_{i=1}^{3} \partial_{t} \mathfrak{q}_{2 j+1}\left(\partial_{t}^{j / 2-1} \lambda^{i}, \partial_{s}^{j-1} k^{i}\right) \\
& +\left.\mathfrak{q}_{2 j+3}\left(\partial_{t}^{j / 2} \lambda^{i}, \partial_{s}^{j} k^{i}\right)\right|_{\text {at the 3-point }} .
\end{aligned}
$$

Now, the key tool to estimate the terms

$$
\int_{\mathbb{T}_{t}} \mathfrak{p}_{2 j+4}\left(\partial_{s}^{j} k\right) d s \text { and }\left.\sum_{i=1}^{3} \mathfrak{q}_{2 j+3}\left(\partial_{t}^{j / 2} \lambda^{i}, \partial_{s}^{j} k^{i}\right)\right|_{\text {at the 3-point }}
$$

are the following Gagliardo-Nirenberg interpolation inequalities (see [2], [8], for instance). 
PROPOSITION 3.11. Let $\gamma$ be a smooth regular curve in $\mathbb{R}^{2}$ with finite length $\mathrm{L}$. If $u$ is a smooth function defined on $\gamma$ and $m \geq 1, p \in[2,+\infty]$, we have the estimates

$$
\left\|\partial_{s}^{n} u\right\|_{L^{p}} \leq C_{n, m, p}\left\|\partial_{s}^{m} u\right\|_{L^{2}}^{\sigma}\|u\|_{L^{2}}^{1-\sigma}+\frac{B_{n, m, p}}{\mathrm{~L}^{m \sigma}}\|u\|_{L^{2}}
$$

for every $n \in\{0, \ldots, m-1\}$ where

$$
\sigma=\frac{n+1 / 2-1 / p}{m}
$$

and the constants $C_{n, m, p}$ and $B_{n, m, p}$ are independent of $\gamma$.

REMARK 3.12. We put in evidence the particular case $p=+\infty$,

$$
\left\|\partial_{s}^{n} u\right\|_{L^{\infty}} \leq C_{n, m}\left\|\partial_{s}^{m} u\right\|_{L^{2}}^{\sigma}\|u\|_{L^{2}}^{1-\sigma}+\frac{B_{n, m}}{\mathrm{~L}^{m \sigma}}\|u\|_{L^{2}} \quad \text { with } \quad \sigma=\frac{n+1 / 2}{m} \text {. }
$$

It clearly follows that for a family of curves with lengths equibounded from below by some positive value, these inequalities hold with uniform constants.

Every monomial of $\mathfrak{p}_{2 j+4}\left(\partial_{s}^{j} k\right)$ is of the form $C \prod_{l=0}^{j}\left(\partial_{s}^{l} k\right)^{\alpha} l$ with $\sum_{l=0}^{j}(l+$ 1) $\alpha_{l}=2 j+4$, then we estimate its integral by means of Hölder inequality,

$$
C \int_{\mathbb{T}_{t}} \prod_{l=0}^{j}\left(\partial_{s}^{l} k\right)^{\alpha_{l}} d s \leq C \prod_{l=0}^{j}\left(\int_{\mathbb{T}_{t}}\left|\partial_{s}^{l} k\right|^{\alpha_{l} \beta_{l}}\right)^{1 / \beta_{l}} d s=C \prod_{l=0}^{j}\left\|\partial_{s}^{l} k\right\|_{L^{\alpha_{l} \beta_{l}}}^{\alpha_{l}}
$$

where the exponents $\beta_{l}$ satisfy $\sum 1 / \beta_{l}=1$ and $\alpha_{l} \beta_{l}>2$ for every $l \in\{0, \ldots, j\}$ such that $\alpha_{l} \neq 0$. These conditions can be fulfilled choosing $\beta_{l}=\frac{2 j+4}{(l+1) \alpha_{l}}$, then $\alpha_{l} \beta_{l}=(2 j+4) /(l+1)>2$ since $l \leq j$ and $\sum 1 / \beta_{l}=\sum_{l=0}^{j}(l+1) \alpha_{l} /(2 j+4)=1$.

Notice that the constant $C$ depends only on the structure of the polynomial $\mathfrak{p}_{2 j+4}\left(\partial_{s}^{j} k\right)$, that is, only on $j \in \mathbb{N}$.

Putting $n=l, m=j+1, p=\alpha_{l} \beta_{l}$ and $u=k$ in inequality (3.7) we get

$$
\left\|\partial_{s}^{l} k\right\|_{L^{\alpha_{l} \beta_{l}}} \leq C_{l}\left(\left\|\partial_{s}^{j+1} k\right\|_{L^{2}}^{\sigma_{l}}\|k\|_{L^{2}}^{1-\sigma_{l}}+\|k\|_{L^{2}}\right)
$$

with $\sigma_{l}=\frac{l+1 / 2-1 /\left(\alpha_{l} \beta_{l}\right)}{j+1}$ for every $l \in\{0, \ldots, j\}$ and the constants $C_{l}$ depend only on the lengths of the curves. $j \in \mathbb{N}$,

Hence, since the number of monomials of $\mathfrak{p}_{2 j+4}\left(\partial_{s}^{j} k\right)$ depends only on

$$
\begin{aligned}
\int_{\mathbb{T}_{t}} \mathfrak{p}_{2 j+4}\left(\partial_{s}^{j} k\right) d s & \leq C \prod_{l=0}^{j}\left(\left\|\partial_{s}^{j+1} k\right\|_{L^{2}}+\|k\|_{L^{2}}\right)^{\sigma_{l} \alpha_{l}}\|k\|_{L^{2}}^{\left(1-\sigma_{l}\right) \alpha_{l}} \\
& \leq C\left(\left\|\partial_{s}^{j+1} k\right\|_{L^{2}}+\|k\|_{L^{2}}\right)^{\sum_{l=0}^{j} \sigma_{l} \alpha_{l}}\|k\|_{L^{2}}^{\sum_{l=0}^{j}\left(1-\sigma_{l}\right) \alpha_{l}}
\end{aligned}
$$


Now we have

$$
\begin{aligned}
\sum_{l=0}^{j} \sigma_{l} \alpha_{l} & =\sum_{l=0}^{j} \alpha_{l} \frac{l+1 / 2-1 /\left(\alpha_{l} \beta_{l}\right)}{j+1}=\sum_{l=0}^{j} \frac{(l+1 / 2) \alpha_{l}-1 / \beta_{l}}{j+1} \\
& =\frac{-1+\sum_{l=0}^{j}(l+1) \alpha_{l}-1 / 2 \alpha_{l}}{j+1}=\frac{2 j+3-1 / 2 \sum_{l=0}^{j} \alpha_{l}}{j+1} \\
& \leq \frac{2 j+3-1 / 2 \sum_{l=0}^{j} \alpha_{l}(l+1) /(j+1)}{j+1}=\frac{2 j+3-(2 j+4) / 2(j+1)}{j+1} \\
& =\frac{2 j+3-1-1 /(j+1)}{j+1}=2-\frac{1}{(j+1)^{2}}<2
\end{aligned}
$$

then, by Young inequality,

$$
\begin{aligned}
& \left(\left\|\partial_{s}^{j+1} k\right\|_{L^{2}}+\|k\|_{L^{2}}\right)^{\sum_{l=0}^{j} \sigma_{l} \alpha_{l}}\|k\|_{L^{2}}^{\sum_{l=0}^{j}\left(1-\sigma_{l}\right) \alpha_{l}} \leq \varepsilon\left(\left\|\partial_{s}^{j+1} k\right\|_{L^{2}}+\|k\|_{L^{2}}\right)^{2} \\
& +C\|k\|_{L^{2}} \frac{\sum_{l=0}^{j}\left(1-\sigma_{l}\right) \alpha_{l}}{2-\sum_{l=0}^{j} \sigma_{l} \alpha_{l}}
\end{aligned}
$$

and this last exponent is equal to

$$
\begin{aligned}
2 \frac{\sum_{l=0}^{j}\left(1-\sigma_{l}\right) \alpha_{l}}{2-\sum_{l=0}^{j} \sigma_{l} \alpha_{l}} & =2 \frac{\sum_{l=0}^{j} \alpha_{l}-\frac{2 j+3-1 / 2 \sum_{l=0}^{j} \alpha_{l}}{j+1}}{2-\frac{2 j+3-1 / 2 \sum_{l=0}^{j} \alpha_{l}}{j+1}} \\
& =2 \frac{(j+1) \sum_{l=0}^{j} \alpha_{l}-2 j-3+1 / 2 \sum_{l=0}^{j} \alpha_{l}}{2 j+2-2 j-3+1 / 2 \sum_{l=0}^{j} \alpha_{l}} \\
& =2 \frac{-2 j-3+(j+3 / 2) \sum_{l=0}^{j} \alpha_{l}}{-1+1 / 2 \sum_{l=0}^{j} \alpha_{l}} \\
& =2(2 j+3) .
\end{aligned}
$$

Choosing a value $\varepsilon>0$ small enough and controlling, via interpolation again, the term $\|k\|_{L^{2}}^{2}$, we conclude

$$
\int_{\mathbb{T}_{t}} \mathfrak{p}_{2 j+4}\left(\partial_{s}^{j} k\right) d s \leq 1 / 4 \int_{\mathbb{T}_{t}}\left|\partial_{s}^{j+1} k\right|^{2} d s+C\left(\int_{\mathbb{T}_{t}} k^{2} d s\right)^{2 j+3}+C
$$

where the constant $C$ depends only on $j \in \mathbb{N}$ and the lengths of the curves of the triod.

The term $\left.\sum_{i=1}^{3} \mathfrak{q}_{2 j+3}\left(\partial_{t}^{j / 2} \lambda^{i}, \partial_{s}^{j} k^{i}\right)\right|_{\text {at the 3-point }}$ can be estimated similarly. Taking into account Remark 3.9, we have $\left.\sum_{i=1}^{3} \mathfrak{q}_{2 j+3}\left(\partial_{t}^{j / 2} \lambda^{i}, \partial_{s}^{j} k^{i}\right)\right|_{\text {at the 3-point }} \leq$ 
$\left\|\mathfrak{p}_{2 j+3}\left(\left|\partial_{s}^{j} k\right|\right)\right\|_{L^{\infty}}$ and this latter can be controlled with a sum of terms like $C \prod_{l=0}^{j}\left\|\partial_{s}^{l} k\right\|_{L^{\infty}}^{\alpha_{l}}$ with $\sum_{l=0}^{j}(l+1) \alpha_{l}=2 j+3$.

Then, we use interpolation inequalities with $p=+\infty$,

$$
\left\|\partial_{s}^{l} k\right\|_{L^{\infty}} \leq C_{l}\left(\left\|\partial_{s}^{j+1} k\right\|_{L^{2}}^{\sigma_{l}}\|k\|_{L^{2}}^{1-\sigma_{l}}+\|k\|_{L^{2}}\right)
$$

with $\sigma_{l}=\frac{l+1 / 2}{j+1}$, hence

$$
\begin{aligned}
& \left.\sum_{i=1}^{3} \mathfrak{q}_{2 j+3}\left(\partial_{t}^{j / 2} \lambda^{i}, \partial_{s}^{j} k^{i}\right)\right|_{\text {at the 3-point }} \leq C \prod_{l=0}^{j}\left(\left\|\partial_{s}^{j+1} k\right\|_{L^{2}}+\|k\|_{L^{2}}\right)^{\sigma_{l} \alpha_{l}}\|k\|_{L^{2}}^{\left(1-\sigma_{l}\right) \alpha_{l}} \\
& \leq C\left(\left\|\partial_{s}^{j+1} k\right\|_{L^{2}}+\|k\|_{L^{2}}\right)^{\sum_{l=0}^{j} \sigma_{l} \alpha_{l}}\|k\|_{L^{2}}^{l=0}\left(1-\sigma_{l}\right) \alpha_{l}
\end{aligned}
$$

and

$$
\begin{aligned}
\sum_{l=0}^{j} \sigma_{l} \alpha_{l} & =\sum_{l=0}^{j} \alpha_{l} \frac{l+1 / 2}{j+1}=\sum_{l=0}^{j} \frac{(l+1 / 2) \alpha_{l}}{j+1} \\
& =\frac{\sum_{l=0}^{j}(l+1) \alpha_{l}-1 / 2 \alpha_{l}}{j+1}=\frac{2 j+3-1 / 2 \sum_{l=0}^{j} \alpha_{l}}{j+1} \\
& \leq \frac{2 j+3-1 / 2 \sum_{l=0}^{j} \alpha_{l}(l+1) /(j+1)}{j+1}=\frac{2 j+3-(2 j+3) / 2(j+1)}{j+1} \\
& =\frac{2 j+3-1-1 / 2(j+1)}{j+1}=2-\frac{1}{2(j+1)^{2}}<2 .
\end{aligned}
$$

As before, by Young inequality,

$$
\begin{aligned}
\left(\left\|\partial_{s}^{j+1} k\right\|_{L^{2}}+\|k\|_{L^{2}}\right)^{\sum_{l=0}^{j} \sigma_{l} \alpha_{l}}\|k\|_{L^{2}}^{\sum_{l=0}^{j}\left(1-\sigma_{l}\right) \alpha_{l}} \leq & \varepsilon\left(\left\|\partial_{s}^{j+1} k\right\|_{L^{2}}+\|k\|_{L^{2}}\right)^{2} \\
& +C\|k\|_{L^{2}} \frac{\sum_{l=0}^{j} \sum_{l=0}^{\left(1-\sigma_{l}\right) \alpha_{l}}}{2-\sum_{l} \alpha_{l}}
\end{aligned}
$$

and the last exponent is again equal to $2(2 j+3)$. Choosing here also a value $\varepsilon>0$ small enough, we get an estimate analogous to the previous one.

Hence, for every even $j \geq 2$ we can finally write

$$
\begin{aligned}
\frac{d}{d t} \int_{\mathbb{T}_{t}}\left|\partial_{s}^{j} k\right|^{2} d s \leq & -1 / 2 \int_{\mathbb{T}_{t}}\left|\partial_{s}^{j+1} k\right|^{2} d s+C\left(\int_{\mathbb{T}_{t}} k^{2} d s\right)^{2 j+3}+C \\
& +\left.\partial_{t} \sum_{i=1}^{3} \mathfrak{q}_{2 j+1}\left(\partial_{t}^{j / 2-1} \lambda^{i}, \partial_{s}^{j-1} k^{i}\right)\right|_{\text {at the 3-point }} \\
\leq & C\left(\int_{\mathbb{T}_{t}} k^{2} d s\right)^{2 j+3}+C \\
& +\left.\partial_{t} \sum_{i=1}^{3} \mathfrak{q}_{2 j+1}\left(\partial_{t}^{j / 2-1} \lambda^{i}, \partial_{s}^{j-1} k^{i}\right)\right|_{\text {at the 3-point }} .
\end{aligned}
$$


Recalling the computation in the special case special case $j=0$, this argument gives the same final estimate without the the last term.

$$
\left|\frac{d}{d t} \int_{\mathbb{T}_{t}} k^{2} d s\right| \leq C\left(\int_{\mathbb{T}_{t}} k^{2} d s\right)^{3}+C .
$$

Integrating (3.9) in time on $[0, t]$ and estimating we get

$$
\begin{aligned}
\int_{\mathbb{T}_{t}}\left|\partial_{s}^{j} k\right|^{2} d s \leq & \int_{\mathbb{T}_{0}}\left|\partial_{s}^{j} k\right|^{2} d s+C \int_{0}^{t}\left(\int_{\mathbb{T}_{\xi}} k^{2} d s\right)^{2 j+3} d \xi+C t \\
& +\sum_{i=1}^{3} \mathfrak{q}_{2 j+1}\left(\partial_{t}^{j / 2-1} \lambda^{i}(0, t), \partial_{s}^{j-1} k^{i}(0, t)\right) \\
& -\mathfrak{q}_{2 j+1}\left(\partial_{t}^{j / 2-1} \lambda^{i}(0,0), \partial_{s}^{j-1} k^{i}(0,0)\right) \\
\leq & C \int_{0}^{t}\left(\int_{\mathbb{T}_{\xi}} k^{2} d s\right)^{2 j+3} d \xi+\left\|\mathfrak{p}_{2 j+1}\left(\left|\partial_{s}^{j-1} k\right|\right)\right\|_{L^{\infty}}+C t+C
\end{aligned}
$$

where in the last passage we used as before Remark 3.9. The constant $C$ depends only on $j \in \mathbb{N}$ and the triod $\mathbb{T}_{0}$.

Interpolating again by means of inequalities (3.8) (we leave the details to the reader), one gets

$$
\left\|\mathfrak{p}_{2 j+1}\left(\left|\partial_{s}^{j-1} k\right|\right)\right\|_{L^{\infty}} \leq 1 / 2\left\|\partial_{s}^{j} k\right\|_{L^{2}}^{2}+C\|k\|_{L^{2}}^{4 j+2} .
$$

Hence, putting all together, for every even $j \in \mathbb{N}$.

$$
\int_{\mathbb{T}_{t}}\left|\partial_{s}^{j} k\right|^{2} d s \leq C \int_{0}^{t}\left(\int_{\mathbb{T}_{\xi}} k^{2} d s\right)^{2 j+3} d \xi+C\left(\int_{\mathbb{T}_{t}} k^{2} d s\right)^{2 j+1}+C t+C
$$

Passing from integral to $L^{\infty}$ estimates by using inequalities (3.8), we have the following proposition.

PROPOSITION 3.13. If the lengths of the three curves are bounded away from zero and the $L^{2}$ norm of $k$ is bounded, uniformly on $[0, T)$, then the curvature of $\mathbb{T}_{t}$ and all its space derivatives of are uniformly bounded in the same time interval by some constants depending only on the $L^{2}$ integrals of the space derivatives of $k$ on the initial triod $\mathbb{T}_{0}$.

Now in the hypotheses of this proposition we deal with $\lambda$ and its derivatives.

At the 3-point $\sum_{i=1}^{3}\left(\lambda^{i}\right)^{2}=\sum_{i=1}^{3}\left(k^{i}\right)^{2}$, hence the squared modulus of the velocity $v^{2}=|\underline{v}|^{2}$ is uniformly bounded at $O$.

Then, since $\gamma_{t}^{i}(1, t)=\underline{v}^{i}(1, t)=0$ for every index $i \in\{1,2,3\}$, by the maximum principle applied to the equation for $v^{2}$,

$$
\partial_{t} v^{2}=\left(v^{2}\right)_{s s}-2 \lambda_{s}^{2}-2 k_{s}^{2}-\lambda\left(v^{2}\right)_{s}+2 v^{2} k^{2}
$$


which follows from equation (2.7)

$$
\partial_{t} \lambda=\lambda_{s s}-\lambda \lambda_{s}-2 k k_{s}+\lambda k^{2}
$$

and equation (2.6), we see that if $v^{2}$ gets larger than its value at the 3-point, then its maximum is taken in the interior of some curve of the triod so, as $k^{2}$ is uniformly bounded,

$$
\partial_{t} v_{\max }^{2} \leq 2 v_{\max }^{2} k_{\max }^{2} \leq C v_{\max }^{2}
$$

Integrating this linear differential inequality, we obtain that $\underline{v}$ and hence $\lambda$ are also uniformly bounded as $k$ and its derivatives in the time interval $[0, T)$.

By Lemma 3.8 and computing like for $k$, we get

$$
\begin{aligned}
\frac{d}{d t} \int_{\mathbb{T}_{t}}\left|\partial_{s}^{j} \lambda\right|^{2} d s= & -2 \int_{\mathbb{T}_{t}}\left|\partial_{s}^{j+1} \lambda\right|^{2} d s-2 \int_{\mathbb{T}_{t}} \lambda \partial_{s}^{j} \lambda \partial_{s}^{j+1} \lambda \\
& +2 k \partial_{s}^{j} \lambda \partial_{s}^{j+1} k d s+\int_{\mathbb{T}_{t}} \mathfrak{p}_{2 j+4}\left(\partial_{s}^{j} \lambda, \partial_{s}^{j} k\right) d s \\
& -\left.2 \sum_{i=1}^{3} \partial_{s}^{j} \lambda^{i} \partial_{s}^{j+1} \lambda^{i}\right|_{\text {at the 3-point }} \\
& +\left.2 \sum_{i=1}^{3} \partial_{s}^{j} \lambda^{i} \partial_{s}^{j+1} \lambda^{i}\right|_{\text {at the point } P^{i}} \\
\leq & -\int_{\mathbb{T}_{t}}\left|\partial_{s}^{j+1} \lambda\right|^{2} d s+\int_{\mathbb{T}_{t}} \mathfrak{p}_{2 j+4}\left(\partial_{s}^{j} \lambda, \partial_{s}^{j} k\right) d s \\
& +\int_{\mathbb{T}_{t}}\left|\partial_{s}^{j+1} k\right|^{2} d s \\
& -\left.2 \sum_{i=1}^{3} \partial_{s}^{j} \lambda^{i} \partial_{s}^{j+1} \lambda^{i}\right|_{\text {at the 3-point }} \\
\leq & -\int_{\mathbb{T}_{t}}\left|\partial_{s}^{j+1} \lambda\right|^{2} d s+\int_{\mathbb{T}_{t}} \mathfrak{r}_{2 j+4}\left(\left|\partial_{s}^{j} \lambda\right|\right)+C \\
& -\left.2 \sum_{i=1}^{3} \partial_{s}^{j} \lambda^{i} \partial_{s}^{j+1} \lambda^{i}\right|_{\text {at the 3-point }}
\end{aligned}
$$

where in the first passage we used Peter-Paul inequality $a b \leq \varepsilon a^{2}+b^{2} / 4 \varepsilon$ with $\varepsilon=1 / 2, a=\partial_{s}^{j+1} \lambda$ and $b=\lambda \partial_{s}^{j} \lambda$ on the first term of the second integral, and with $\varepsilon=1 / 2, a=\partial_{s}^{j+1} k$ and $b=k \partial_{s}^{j} \lambda$ on the second term of the second integral. Then, we summed and absorbed the terms without $(j+1)$-th derivatives into $\mathfrak{p}_{2 j+4}\left(\partial_{s}^{j} \lambda, \partial_{s}^{j} k\right)$.

In the second passage, using Young inequality, we "separated" in all the monomials of $\mathfrak{p}_{2 j+4}\left(\partial_{s}^{j} \lambda, \partial_{s}^{j} k\right)$ the derivatives of $\lambda$ and $k$, controlling them with 
$\mathfrak{r}_{2 j+4}\left(\left|\partial_{s}^{j} \lambda\right|\right)+\mathfrak{r}_{2 j+4}\left(\left|\partial_{s}^{j} k\right|\right)$, where $\mathfrak{r}_{2 j+4}$ denotes a "polynomial" (similar to $\mathfrak{p}$ and $\mathfrak{q}$ ) with real exponents all greater or equal than 1 .

Then we estimated the term $\mathfrak{r}_{2 j+4}\left(\left|\partial_{s}^{j} k\right|\right)$ with some constant, as we know that $k$ and its space derivatives are bounded. Notice that the number of monomials of $\mathfrak{p}_{2 j+4}\left(\partial_{s}^{j} \lambda, \partial_{s}^{j} k\right)$ depends only on $j \in \mathbb{N}$.

Finally, the contributions of the end points $P^{i}$ vanish by Lemma 3.10, since at least one of the two derivatives of $\lambda$ is even.

We estimate by interpolation, exactly like for $k$, the term $\int_{\mathbb{T}_{t}} \mathfrak{r}_{2 j+4}\left(\left|\partial_{s}^{j} \lambda\right|\right) d s$ with a small fraction of the "good" term $\int_{\mathbb{T}_{t}}\left|\partial_{s}^{j+1} \lambda\right|^{2} d s$ and a possibly large multiple of $\left(\int_{\mathbb{T}_{t}} \lambda^{2} d s\right)^{2 j+3}$, which is bounded by the argument above.

Hence, it only remains to control the 3-point term

$$
-\left.2 \sum_{i=1}^{3} \partial_{s}^{j} \lambda^{i} \partial_{s}^{j+1} \lambda^{i}\right|_{\text {at the 3-point }} \leq\left. C \sum_{i=1}^{3}\left|\partial_{s}^{j} \lambda^{i}\right|\left|\partial_{s}^{j+1} \lambda^{i}\right|\right|_{\text {at the 3-point }} .
$$

Now, if $j \in \mathbb{N}$ is odd, by the second formula of Lemma 3.8,

$$
\partial_{s}^{j+1} \lambda^{i}=\partial_{t}^{(j+1) / 2} \lambda^{i}+\mathfrak{p}_{j+2}\left(\partial_{s}^{j} \lambda^{i}, \partial_{s}^{j} k^{i}\right)
$$

thus, recalling that $\left.\left|\partial_{t}^{(j+1) / 2} \lambda^{i}\right|\right|_{\text {at the 3-point }} \leq\left\|\mathfrak{p}_{j+2}\left(\left|\partial_{s}^{j+1} k\right|\right)\right\|_{L^{\infty}}$ by Remark 3.9,

$$
\begin{aligned}
\left.\left|\partial_{s}^{j} \lambda^{i}\right|\left|\partial_{s}^{j+1} \lambda^{i}\right|\right|_{\text {at the 3-point }} \leq & \left.\left|\partial_{s}^{j} \lambda^{i}\right|\left(\left|\partial_{t}^{(j+1) / 2} \lambda^{i}\right|+\left|\mathfrak{p}_{j+2}\left(\partial_{s}^{j} \lambda^{i}, \partial_{s}^{j} k^{i}\right)\right|\right)\right|_{\text {at the 3-point }} \\
\leq & \left\|\partial_{s}^{j} \lambda\right\|_{L^{\infty}}\left\|\mathfrak{p}_{j+2}\left(\left|\partial_{s}^{j+1} k\right|\right)\right\|_{L^{\infty}}+\left\|\mathfrak{p}_{2 j+3}\left(\left|\partial_{s}^{j} \lambda\right|,\left|\partial_{s}^{j} k\right|\right)\right\|_{L^{\infty}} \\
\leq & \left\|\partial_{s}^{j} \lambda\right\|_{L^{\infty}}^{\frac{2 j+3}{j+1}}+\left\|\mathfrak{p}_{j+2}\left(\left|\partial_{s}^{j+1} k\right|\right)\right\|_{L^{\infty}}^{\frac{2 j+3}{j+2}} \\
& +\left\|\mathfrak{p}_{2 j+3}\left(\left|\partial_{s}^{j} \lambda\right|,\left|\partial_{s}^{j} k\right|\right)\right\|_{L^{\infty}} \\
\leq & \left\|\mathfrak{r}_{2 j+3}\left(\left|\partial_{s}^{j} \lambda\right|\right)\right\|_{L^{\infty}}+C+\left\|\mathfrak{p}_{2 j+3}\left(\left|\partial_{s}^{j} \lambda\right|,\left|\partial_{s}^{j} k\right|\right)\right\|_{L^{\infty}} \\
\leq & \left\|\mathfrak{r}_{2 j+3}\left(\left|\partial_{s}^{j} \lambda\right|\right)\right\|_{L^{\infty}}+C+\| \mathfrak{r}_{2 j+3}\left(\left|\partial_{s}^{j} \lambda\right|\right) \\
& +\mathfrak{r}_{2 j+3}\left(\left|\partial_{s}^{j} k\right|\right) \|_{L^{\infty}} \\
\leq & \left\|\mathfrak{r}_{2 j+3}\left(\left|\partial_{s}^{j} \lambda\right|\right)\right\|_{L^{\infty}}+C
\end{aligned}
$$

where we used Young inequality and the fact that $\left\|\mathfrak{p}_{j+2}\left(\left|\partial_{s}^{j+1} k\right|\right)\right\|_{L^{\infty}}$ is uniformly bounded.

Moreover, we separated, as before, the derivatives of $\lambda$ and $k$ in every monomial of $\mathfrak{p}_{2 j+3}\left(\partial_{s}^{j} \lambda, \partial_{s}^{j} k\right)$, hence estimating them with $\mathfrak{r}_{2 j+3}\left(\left|\partial_{s}^{j} \lambda\right|\right)+\mathfrak{r}_{2 j+3}\left(\left|\partial_{s}^{j} k\right|\right)$. Finally, we controlled the $k$-terms with some constants and we can now interpolate the $\lambda$-terms like we did for $k$, since these latter do not contain $(j+1)$-th space derivatives of $\lambda$.

Hence, coming back to computation (3.11), we conclude that for every odd $j \in \mathbb{N}$

$$
\frac{d}{d t} \int_{\mathbb{T}_{t}}\left|\partial_{s}^{j} \lambda\right|^{2} d s \leq C<+\infty
$$

for a constant $C$ depending only on $j \in \mathbb{N}$ and $\mathbb{T}_{0}$. 
Like for $k$, since we know that $\|\lambda\|_{L^{2}}$ is bounded, passing from integral to $L^{\infty}$ estimates by means of inequalities (3.8), we obtain that also all the space derivatives of $\lambda$ are uniformly bounded in $[0, T)$.

Then, we can bound from above and positively from below the term $\left|\gamma_{x}\right|$ at the denominator in the evolution equation (2.2).

$$
\partial_{t} \log \left|\gamma_{x}(x, t)\right|=\frac{\left\langle\gamma_{x} \mid \gamma_{x t}\right\rangle}{\left|\gamma_{x}\right|^{2}}=\left\langle\tau \mid \partial_{s}(\lambda \tau+k \nu)\right\rangle=\lambda_{s}-k^{2} \leq C<+\infty
$$

for a constant $C$ independent of $x \in[0,1]$ and $t \in[0, T)$. This implies that $\left|\gamma_{x}\right|$ is bounded from above and away from zero, uniformly in space and time as

$$
|\log | \gamma_{x}(x, t)|| \leq \int_{0}^{t}\left|\partial_{t} \log \right| \gamma_{x}(x, \xi)|| d \xi \leq C t \leq C T<+\infty .
$$

Since $\left|\gamma_{x}\right|$ is uniformly bounded and $\partial_{x}=\left|\gamma_{x}\right| \partial_{s}$, by using the evolution equation (2.2), it follows that all the mixed derivatives in $x$ and $t$ of $\gamma^{i}$ for every $i \in\{1,2,3\}$ are uniformly bounded in $[0,1] \times[0, T)$.

Proposition 3.14. If $\mathbb{T}_{t}$ is a smooth evolution of the initial triod $\mathbb{T}_{0}=\cup_{i=1}^{3} \sigma^{i}$ such that the lengths of the three curves are uniformly bounded away from zero and the $L^{2}$ norm of the curvature is uniformly bounded by some constants in the time interval $[0, T)$, then

- all the derivatives in space and time of $k$ and $\lambda$ are uniformly bounded in $[0,1] \times[0, T)$,

- all the derivatives in space and time of the curves $\gamma^{i}(x, t)$ are uniformly bounded in $[0,1] \times[0, T)$,

- the quantities $\left|\gamma_{x}^{i}(x, t)\right|$ are uniformly bounded from above and away from zero in $[0,1] \times[0, T)$.

All the bounds depend only on the uniform controls on $k$ and the lengths of the curves, and on the $L^{\infty}$ norms of the derivatives of the maps $\sigma^{i}$ composing the initial triod $\mathbb{T}_{0}$.

Now, we work out a second family of estimates where everything is controlled only by the $L^{2}$ norm of the curvature and the inverses of the lengths of the three curves at time zero.

As before we consider the smooth evolution $\mathbb{T}_{t}$ of a smooth triod $\mathbb{T}_{0}$ in the time interval $[0, T)$.

Proposition 3.15. For every $M>0$ there exists a time $T_{M} \in(0, T)$ such that if the $L^{2}$ norm of the curvature and the inverses of the lengths of the three curves of $\mathbb{T}_{0}$ are bounded by $M$, then the $L^{2}$ norm of $k$ and the inverses of the lengths of the curves of $\mathbb{T}_{t}$ are smaller than $2 M^{2}+6 M$, for every time $t \in\left[0, T_{M}\right]$. 
PROOF. The evolution equations for the lengths of the three curves are given by $\frac{d L^{i}(t)}{d t}=-\lambda^{i}(0, t)-\int_{\gamma^{i}(\cdot, t)} k^{2} d s$ (Proposition 3.2), then recalling computation (3.5), we have

$$
\begin{aligned}
& \frac{d}{d t}\left(\int_{\mathbb{T}_{t}} k^{2} d s+\sum_{i=1}^{3} \frac{1}{L^{i}}\right) \leq-2 \int_{\mathbb{T}_{t}} k_{s}^{2} d s+\int_{\mathbb{T}_{t}} k^{4} d s+C\|k\|_{L^{\infty}}^{3}-\sum_{i=1}^{3} \frac{1}{\left(L^{i}\right)^{2}} \frac{d L}{d t} \\
& \quad=-2 \int_{\mathbb{T}_{t}} k_{s}^{2} d s+\int_{\mathbb{T}_{t}} k^{4} d s+C\|k\|_{L^{\infty}}^{3}+\sum_{i=1}^{3} \frac{\lambda^{i}(0, t)+\int_{\gamma^{i}(\cdot, t)} k^{2} d s}{\left(L^{i}\right)^{2}} \\
& \quad \leq-2 \int_{\mathbb{T}_{t}} k_{s}^{2} d s+\int_{\mathbb{T}_{t}} k^{4} d s+C\|k\|_{L^{\infty}}^{3}+C \sum_{i=1}^{3} \frac{\|k\|_{L^{\infty}}}{\left(L^{i}\right)^{2}}+\sum_{i=1}^{3} \frac{\int_{\mathbb{T}_{t}} k^{2} d s}{\left(L^{i}\right)^{2}} \\
& \quad \leq-2 \int_{\mathbb{T}_{t}} k_{s}^{2} d s+\int_{\mathbb{T}_{t}} k^{4} d s+C\|k\|_{L^{\infty}}^{3}+C\left(\int_{\mathbb{T}_{t}} k^{2} d s\right)^{3}+C \sum_{i=1}^{3} \frac{1}{\left(L^{i}\right)^{3}}
\end{aligned}
$$

where we used Young inequality in the last passage.

Interpolating as before (and applying again Young inequality) but keeping now in evidence the terms depending on $L^{i}$ in inequalities (3.7) and (3.8), we obtain

$$
\begin{aligned}
\frac{d}{d t}\left(\int_{\mathbb{T}_{t}} k^{2} d s+\sum_{i=1}^{3} \frac{1}{L^{i}}\right) \leq & -\int_{\mathbb{T}_{t}} k_{s}^{2} d s+C\left(\int_{\mathbb{T}_{t}} k^{2} d s\right)^{3}+C \sum_{i=1}^{3} \frac{\left(\int_{\mathbb{T}_{t}} k^{2} d s\right)^{2}}{L^{i}} \\
& +C \sum_{i=1}^{3} \frac{\left(\int_{\mathbb{T}_{t}} k^{2} d s\right)^{3 / 2}}{\left(L^{i}\right)^{3 / 2}}+C \sum_{i=1}^{3} \frac{1}{\left(L^{i}\right)^{3}} \\
\leq & C\left(\int_{\mathbb{T}_{t}} k^{2} d s\right)^{3}+C \sum_{i=1}^{3} \frac{1}{\left(L^{i}\right)^{3}} \\
\leq & C\left(\int_{\mathbb{T}_{t}} k^{2} d s+\sum_{i=1}^{3} \frac{1}{L^{i}}\right)^{3}
\end{aligned}
$$

with a universal constant $C$ independent of the triods.

This means that the function $f(t)=\int_{\mathbb{T}_{t}} k^{2} d s+\sum_{i=1}^{3} \frac{1}{L^{i}(t)}$ satisfies the differential inequality $f^{\prime} \leq C f^{3}$, hence, after integration the thesis follows.

By means of this proposition we can strengthen the conclusion of Proposition 3.14.

COROLLARY 3.16. In the hypothesis of the previous proposition, in the time interval $\left[0, T_{M}\right]$ all the bounds in Proposition 3.14 depends only on the $L^{2}$ norm of the curvature and the lengths of the curves of $\mathbb{T}_{0}=\cup_{i=1}^{3} \sigma^{i}$ and on the $L^{\infty}$ norms of the derivatives of the maps $\sigma^{i}$. 
Then, from now on we assume that the $L^{2}$ norm of the curvature and the inverses of the lengths of the three curves are bounded in the interval $\left[0, T_{M}\right]$.

Before dealing with the general estimate, we work out a special case as an example.

By means of computations (3.4), we obtain

$$
\begin{aligned}
& \frac{d}{d t} \int_{\mathbb{T}_{t}} k^{2}+t k_{s}^{2}+\frac{t^{2} k_{s s}^{2}}{2} d s=-2 \int_{\mathbb{T}_{t}} k_{s}^{2}+t k_{s s}^{2}+\frac{t^{2} k_{s s s}^{2}}{2} d s+\int_{\mathbb{T}_{t}} k_{s}^{2}+t k_{s s}^{2} d s \\
&+\int_{\mathbb{T}_{t}} k^{4}+t \mathfrak{p}_{6}\left(k_{s}\right)+t^{2} \mathfrak{p}_{8}\left(k_{s s}\right) d s \\
&+\sum_{i=1}^{3} \lambda^{i}\left(k^{i}\right)^{2}-t\left[2 k_{s}^{i} k_{s s}^{i}+\lambda^{i}\left(k_{s}^{i}\right)^{2}\right] \\
&-t^{2}\left[2 k_{s s}^{i} k_{s s s}^{i}+\lambda^{i}\left(k_{s s}^{i}\right)^{2}\right] /\left.2\right|_{\text {at the 3-point }} \\
&=-\int_{\mathbb{T}_{t}} k_{s}^{2}+t k_{s s}^{2}+t^{2} k_{s s s}^{2} d s \\
&+\int_{\mathbb{T}_{t}} k^{4}+t \mathfrak{p}_{6}\left(k_{s}\right)+t^{2} \mathfrak{p}_{8}\left(k_{s s}\right) d s \\
&+\sum_{i=1}^{3} \lambda^{i}\left(k^{i}\right)^{2}-t\left[2 k_{s}^{i} k_{s s}^{i}+\lambda^{i}\left(k_{s}^{i}\right)^{2}\right] \\
&-t^{2}\left[2 k_{s s}^{i} k_{s s s}^{i}+\lambda^{i}\left(k_{s s}^{i}\right)^{2}\right] /\left.2\right|_{\text {at the 3-point }}
\end{aligned}
$$

Estimating, as we did before in order to get inequality (3.9), the terms coming from the integrals of $k^{2}$ and $t^{2} k_{s s}^{2}$ (the even terms) we get

$$
\begin{aligned}
\frac{d}{d t} \int_{\mathbb{T}_{t}} k^{2}+t k_{s}^{2}+t^{2} k_{s s}^{2} / 2 d s \leq & -1 / 2 \int_{\mathbb{T}_{t}} k_{s}^{2}+t k_{s s}^{2}+t^{2} k_{s s s}^{2} d s \\
& +\int_{\mathbb{T}_{t}} t \mathfrak{p}_{6}\left(k_{s}\right) d s-t \sum_{i=1}^{3} 2 k_{s}^{i} k_{s s}^{i}+\left.\lambda^{i}\left(k_{s}^{i}\right)^{2}\right|_{\text {at the 3-point }} \\
& +C\left(\int_{\mathbb{T}_{t}} k^{2} d s\right)^{3}+C t^{2}\left(\int_{\mathbb{T}_{t}} k^{2} d s\right)^{7}+C\left(1+t^{2}\right) \\
& +\left.t^{2} \partial_{t} \sum_{i=1}^{3} \mathfrak{q}_{5}\left(\lambda^{i}, k_{s}^{i}\right)\right|_{\text {at the 3-point }} .
\end{aligned}
$$

The integral term $\int_{\mathbb{T}_{t}} t \mathfrak{p}_{6}\left(k_{s}\right) d s$ and the term $-\left.t \sum_{i=1}^{3} \lambda^{i}\left(k_{s}^{i}\right)^{2}\right|_{\text {at the 3-point }}$, which is of type $t \mathfrak{q}_{5}\left(\lambda^{i}, k_{s}^{i}\right)$, can be estimated by means of interpolation inequalities 
like in the even derivative case with a small fraction of the term $t \int_{\mathbb{T}_{t}} k_{s s}^{2}$ and a possibly large multiple of $t\left(\int_{\mathbb{T}_{t}} k^{2} d s\right)^{5}$. The same holds for the term $-\left.2 t \sum_{i=1}^{3} \mathfrak{q}_{5}\left(\lambda^{i}, k_{s}^{i}\right)\right|_{\text {at the 3-point }}$ arising by putting $t^{2}$ inside the time derivative of the last sum. Finally since the $L^{2}$ norm of $k$ is bounded by some constant and $t \leq T_{M}$, we conclude

$$
\begin{aligned}
\frac{d}{d t} \int_{\mathbb{T}_{t}} k^{2}+t k_{s}^{2}+t^{2} k_{s s}^{2} / 2 d s \leq & -1 / 4 \int_{\mathbb{T}_{t}} k_{s}^{2}+t k_{s s}^{2}+t^{2} k_{s s s}^{2} d s+C \\
& +\left.\partial_{t} \sum_{i=1}^{3} t^{2} \mathfrak{q}_{5}\left(\lambda^{i}, k_{s}^{i}\right)\right|_{\text {at the 3-point }} \\
& -\left.2 t \sum_{i=1}^{3} k_{s}^{i} k_{s s}^{i}\right|_{\text {at the 3-point }}
\end{aligned}
$$

The last term is the only one which needs a special analysis, so we deal with it in the general case.

Considering now $j \in \mathbb{N}$ even and following exactly the same line, if we differentiate the function

$$
\int_{\mathbb{T}_{t}} k^{2}+t k_{s}^{2}+\frac{t^{2} k_{s s}^{2}}{2 !}+\cdots+\frac{t^{j}\left|\partial_{s}^{j} k\right|^{2}}{j !} d s
$$

and we estimate as above, we obtain

$$
\begin{aligned}
\frac{d}{d t} \int_{\mathbb{T}_{t}} k^{2} & +t k_{s}^{2}+\frac{t^{2} k_{s s}^{2}}{2 !}+\cdots+\frac{t^{j}\left|\partial_{s}^{j} k\right|^{2}}{j !} d s \\
\leq & -\varepsilon \int_{\mathbb{T}_{t}} k_{s}^{2}+t k_{s s}^{2}+t^{2} k_{s s s}^{2}+\cdots+t^{j}\left|\partial_{s}^{j+1} k\right|^{2} d s+C \\
& +\partial_{t} \sum_{i=1}^{3} t^{2} \mathfrak{q}_{5}\left(\lambda^{i}, k_{s}^{i}\right) \\
& +t^{4} \mathfrak{q}_{9}\left(\partial_{t} \lambda^{i}, k_{s s s}^{i}\right)+\cdots+\left.t^{j} \mathfrak{q}_{2 j+1}\left(\partial_{t}^{j / 2-1} \lambda^{i}, \partial_{s}^{j-1} k^{i}\right)\right|_{\text {at the 3-point }} \\
& +C \sum_{i=1}^{3} t k_{s}^{i} k_{s s}^{i}+t^{3} k_{s s s}^{i} k_{s s s s}^{i}+\cdots+\left.t^{j-1} \partial_{s}^{j-1} k^{i} \partial_{s}^{j} k^{i}\right|_{\text {at the 3-point }}
\end{aligned}
$$

in the time interval $\left[0, T_{M}\right]$, where $\varepsilon>0$ and $C$ are two constants depending only on the $L^{2}$ norm of the curvature and the inverses of the lengths of the three curves of $\mathbb{T}_{0}$. 
We deal now with the terms $\left.\sum_{i=1}^{3} t^{h-1} \partial_{s}^{h-1} k^{i} \partial_{s}^{h} k^{i}\right|_{\text {at the 3-point }}$ with $h \in$ $\{2, \ldots, j\}$ even (notice that this family contains the term $t k_{s}^{i} k_{s s}^{i}$ of the case $j=2$ above). By formulas (3.3) we have

$$
\begin{aligned}
\partial_{s}^{h-1} k \cdot & \partial_{s}^{h} k \\
= & {\left[\partial_{t}^{h / 2-1} k_{s}+\mathfrak{q}_{h}\left(\partial_{t}^{h / 2-2} \lambda, \partial_{s}^{h-2} k\right)\right] \cdot\left[\partial_{t}^{h / 2} k+\mathfrak{q}_{h+1}\left(\partial_{t}^{h / 2-1} \lambda, \partial_{s}^{h-1} k\right)\right] } \\
= & {\left[\partial_{t}^{h / 2-1}\left(k_{s}+\lambda k\right)+\mathfrak{q}_{h}\left(\partial_{t}^{h / 2-1} \lambda, \partial_{s}^{h-2} k\right)\right] \cdot\left[\partial_{t}^{h / 2} k+\mathfrak{q}_{h+1}\left(\partial_{t}^{h / 2-1} \lambda, \partial_{s}^{h-1} k\right)\right] } \\
= & \partial_{t}^{h / 2-1}\left(k_{s}+\lambda k\right) \cdot \partial_{t}^{h / 2} k+\partial_{t}^{h / 2-1}\left(k_{s}+\lambda k\right) \cdot \mathfrak{q}_{h+1}\left(\partial_{t}^{h / 2-1} \lambda, \partial_{s}^{h-1} k\right) \\
& +\mathfrak{q}_{h}\left(\partial_{t}^{h / 2-1} \lambda, \partial_{s}^{h-2} k\right) \cdot \mathfrak{q}_{h+1}\left(\partial_{t}^{h / 2-1} \lambda, \partial_{s}^{h-1} k\right)+\partial_{t}^{h / 2} k \cdot \mathfrak{q}_{h}\left(\partial_{t}^{h / 2-1} \lambda, \partial_{s}^{h-2} k\right) \\
= & \partial_{t}^{h / 2-1}\left(k_{s}+\lambda k\right) \cdot \partial_{t}^{h / 2} k+\mathfrak{q}_{2 h+1}\left(\partial_{t}^{h / 2-1} \lambda, \partial_{s}^{h-1} k\right) \\
& +\partial_{t}^{h / 2} k \cdot \mathfrak{q}_{h}\left(\partial_{t}^{h / 2-1} \lambda, \partial_{s}^{h-2} k\right) \\
= & \partial_{t}^{h / 2-1}\left(k_{s}+\lambda k\right) \cdot \partial_{t}^{h / 2} k+\mathfrak{q}_{2 h+1}\left(\partial_{t}^{h / 2-1} \lambda, \partial_{s}^{h-1} k\right) \\
& +\left[\partial_{s}^{h} k+\mathfrak{q}_{h+1}\left(\partial_{t}^{h / 2-1} \lambda, \partial_{s}^{h-1} k\right)\right] \cdot \mathfrak{q}_{h}\left(\partial_{t}^{h / 2-1} \lambda, \partial_{s}^{h-2} k\right) \\
= & \partial_{t}^{h / 2-1}\left(k_{s}+\lambda k\right) \cdot \partial_{t}^{h / 2} k+\mathfrak{q}_{2 h+1}\left(\partial_{t}^{h / 2-1} \lambda, \partial_{s}^{h-1} k\right) \\
& +\partial_{s}^{h} k \cdot \mathfrak{q}_{h}\left(\partial_{t}^{h / 2-1} \lambda, \partial_{s}^{h-2} k\right)
\end{aligned}
$$

and since we are summing at the 3-point, the first product is zero by relations (2.10) and, by Remark 3.9, we get

$$
\begin{aligned}
\left.\sum_{i=1}^{3} t^{h-1} \partial_{s}^{h-1} k^{i} \partial_{s}^{h} k^{i}\right|_{\text {at the 3-point }} & =\sum_{i=1}^{3} t^{h-1} \mathfrak{q}_{2 h+1}\left(\partial_{t}^{h / 2-1} \lambda^{i}, \partial_{s}^{h-1} k^{i}\right) \\
& +\left.t^{h-1} \partial_{s}^{h} k^{i} \cdot \mathfrak{q}_{h}\left(\partial_{t}^{h / 2-1} \lambda^{i}, \partial_{s}^{h-2} k^{i}\right)\right|_{\text {at the 3-point }} \\
\leq & t^{h-1}\left\|\mathfrak{p}_{2 h+1}\left(\left|\partial_{s}^{h-1} k\right|\right)\right\|_{L^{\infty}} \\
& +t^{h-1}\left\|\partial_{s}^{h} k\right\|_{L^{\infty}}\left\|\mathfrak{p}_{h}\left(\left|\partial_{s}^{h-2} k\right|\right)\right\|_{L^{\infty}} .
\end{aligned}
$$

The term $t^{h-1}\left\|\mathfrak{p}_{2 h+1}\left(\left|\partial_{s}^{h-1} k\right|\right)\right\|_{L^{\infty}}$ is controlled as before by a small fraction of the term $t^{h-1} \int_{\mathbb{T}_{t}}\left|\partial_{s}^{h} k\right|^{2} d s$ and a possibly large multiple of $t^{h-1}$ times some power of the $L^{2}$ norm of $k$ (which is bounded), whereas $t^{h-1}\left\|\partial_{s}^{h} k\right\|_{L^{\infty}}\left\|\mathfrak{p}_{h}\left(\left|\partial_{s}^{h-2} k\right|\right)\right\|_{L^{\infty}}$ is the critical term. 
Again by means of interpolation inequalities (3.8) we have

$$
\begin{aligned}
& \left\|\partial_{s}^{h} k\right\|_{L^{\infty}} \leq C\left\|\partial_{s}^{h+1} k\right\|_{L^{2}}^{1 / 2}\left\|\partial_{s}^{h} k\right\|_{L^{2}}^{1 / 2}+C\left\|\partial_{s}^{h} k\right\|_{L^{2}} \\
& \leq C\left(\left\|\partial_{s}^{h+1} k\right\|_{L^{2}}^{1 / 2}+C\left\|\partial_{s}^{h} k\right\|_{L^{2}}^{1 / 2}\right)\left\|\partial_{s}^{h} k\right\|_{L^{2}}^{1 / 2} \\
& \left\|\mathfrak{p}_{h}\left(\left|\partial_{s}^{h-2} k\right|\right)\right\|_{L^{\infty}} \leq C\left\|\partial_{s}^{h+1} k\right\|_{L^{2}}^{\sigma}\|k\|_{L^{2}}^{\sum_{l=0}^{h-2} \alpha_{l}-\sigma}+C\|k\|_{L^{2}}^{h} \leq C\left\|\partial_{s}^{h+1} k\right\|_{L^{2}}^{\sigma}+C \\
& \leq C\left(\left\|\partial_{s}^{h+1} k\right\|_{L^{2}}^{2}+C\right)^{\sigma / 2} \\
& \left\|\partial_{s}^{h} k\right\|_{L^{2}} \leq C\left\|\partial_{s}^{h+1} k\right\|_{L^{2}}^{h /(h+1)}\|k\|_{L^{2}}^{1 /(h+1)}+C\|k\|_{L^{2}} \\
& \leq C\left\|\partial_{s}^{h+1} k\right\|_{L^{2}}+C
\end{aligned}
$$

with $\sigma=\frac{\sum_{l=0}^{h-2}(l+1 / 2) \alpha_{l}}{h+1}$ as every monomial of $\mathfrak{p}_{h}\left(\left|\partial_{s}^{h-2} k\right|\right)$ is less than $C \prod_{l=0}^{h-2}\left|\partial_{s}^{l} k\right|^{\alpha_{l}}$.

Hence, putting together the first and third inequalities above,

$$
\begin{aligned}
\left\|\partial_{s}^{h} k\right\|_{L^{\infty}} & \leq C\left(\left\|\partial_{s}^{h+1} k\right\|_{L^{2}}^{2}+C\left\|\partial_{s}^{h} k\right\|_{L^{2}}^{2}\right)^{1 / 4}\left\|\partial_{s}^{h} k\right\|_{L^{2}}^{1 / 2} \\
& \leq C\left(\left\|\partial_{s}^{h+1} k\right\|_{L^{2}}^{2}+C\right)^{1 / 4}\left\|\partial_{s}^{h} k\right\|_{L^{2}}^{1 / 2}
\end{aligned}
$$

and multiplying this last with the second we get

$$
\begin{aligned}
& t^{h-1}\left\|\partial_{s}^{h} k\right\|_{L^{\infty}}\left\|\mathfrak{p}_{h}\left(\left|\partial_{s}^{h-2} k\right|\right)\right\|_{L^{\infty}} \\
& \leq C t^{h-1}\left(\left\|\partial_{s}^{h+1} k\right\|_{L^{2}}^{2}+C\right)^{\sigma / 2+1 / 4}\left\|\partial_{s}^{h} k\right\|_{L^{2}}^{1 / 2} \\
& =C t^{h-1}\left(\int_{\mathbb{T}_{t}}\left|\partial_{s}^{h+1} k\right|^{2} d s+C\right)^{\frac{\sum_{l=0}^{h-2}(l+1 / 2) \alpha_{l}}{2(h+1)}+\frac{1}{4}}\left(\int_{\mathbb{T}_{t}}\left|\partial_{s}^{h} k\right|^{2} d s\right)^{1 / 4} \\
& =C \frac{\left(t^{h} \int_{\mathbb{T}_{t}}\left|\partial_{s}^{h+1} k\right|^{2} d s+C t^{h}\right)^{\frac{\sum_{l=0}^{h-2}(l+1 / 2) \alpha_{l}}{2(h+1)}+\frac{1}{4}}\left(t^{h-1} \int_{\mathbb{T}_{t}}\left|\partial_{s}^{h} k\right|^{2} d s\right)^{1 / 4}}{t^{1-h} \cdot t^{h \frac{\sum_{l=0}^{h-2}(l+1 / 2) \alpha_{l}}{2(h+1)}+\frac{h}{4}} \cdot t^{\frac{h-1}{4}}} \\
& =C \frac{\left(t^{h} \int_{\mathbb{T}_{t}}\left|\partial_{s}^{h+1} k\right|^{2} d s+C t^{h}\right)^{\frac{\sum_{l=0}^{h-2}(l+1 / 2) \alpha_{l}}{2(h+1)}+\frac{1}{4}}\left(t^{h-1} \int_{\mathbb{T}_{t}}\left|\partial_{s}^{h} k\right|^{2} d s\right)^{1 / 4}}{t^{3 / 4-h / 2+h} \frac{\sum_{l=0}^{h-2}(l+1 / 2) \alpha_{l}}{2(h+1)}} .
\end{aligned}
$$

Now, applying Young inequality, if we elevate to $\left(\frac{\sum_{l=0}^{h-2}(l+1 / 2) \alpha_{l}}{2(h+1)}+\frac{1}{4}\right)^{-1}$ the first term at the numerator, to 4 the second, as $\sum_{l=0}^{h-2}(l+1) \alpha_{l}=h$, it follows 
that the exponent which, at the end, goes on $t$ at the denominator is

$$
\begin{aligned}
\theta_{h} & =\left(3 / 4-h / 2+h \frac{\sum_{l=0}^{h-2}(l+1 / 2) \alpha_{l}}{2(h+1)}\right)\left(1-1 / 4-\frac{\sum_{l=0}^{h-2}(l+1 / 2) \alpha_{l}}{2(h+1)}-1 / 4\right)^{-1} \\
& =\left(3 / 4-h / 2+h \frac{h-1 / 2 \sum_{l=0}^{h-2} \alpha_{l}}{2(h+1)}\right)\left(1 / 2-\frac{h-1 / 2 \sum_{l=0}^{h-2} \alpha_{l}}{2(h+1)}\right)^{-1} \\
& =\frac{3 h+3-2 h^{2}-2 h+2 h^{2}-h \sum_{l=0}^{h-2} \alpha_{l}}{4(h+1)} \cdot \frac{4(h+1)}{2 h+2-2 h+\sum_{l=0}^{h-2} \alpha_{l}} \\
& =\frac{h+3-h \sum_{l=0}^{h-2} \alpha_{l}}{2+\sum_{l=0}^{h-2} \alpha_{l}}=\frac{3 h+3}{2+\sum_{l=0}^{h-2} \alpha_{l}}-h
\end{aligned}
$$

and since $\sum_{l=0}^{h-2} \alpha_{l} \geq \sum_{l=0}^{h-2} \alpha_{l} \frac{l+1}{h-1}=\frac{h}{h-1}=1+1 /(h-1)$, we have

$$
\theta_{h} \leq \frac{2 h-3}{3 h-2}=1-\frac{h+1}{3 h-2}<1 \quad \text { for every even } h \geq 2 \text {. }
$$

Thus,

$$
\begin{aligned}
\left.\sum_{i=1}^{3} t^{h-1} \partial_{s}^{h-1} k^{i} \partial_{s}^{h} k^{i}\right|_{\text {at the 3-point }} & \leq \varepsilon_{h} / 2\left(t^{h} \int_{\mathbb{T}_{t}}\left|\partial_{s}^{h+1} k\right|^{2} d s+t^{h-1} \int_{\mathbb{T}_{t}}\left|\partial_{s}^{h} k\right|^{2} d s+C t^{h}\right) \\
& +C / t^{\theta h}
\end{aligned}
$$

with $\theta_{h}<1$ and $\varepsilon_{h}>0$ which can be chosen arbitrarily small.

We apply this argument for every even $h$ from 2 to $j$, choosing accurately the values $\varepsilon_{j}>0$.

Hence, we can continue estimate (3.12) as follows,

$$
\begin{aligned}
& \frac{d}{d t} \int_{\mathbb{T}_{t}} k^{2}+t k_{s}^{2}+\frac{t^{2} k_{s s}^{2}}{2 !}+\cdots+\frac{t^{j}\left|\partial_{s}^{j} k\right|^{2}}{j !} d s \\
& \leq-\varepsilon / 2 \int_{\mathbb{T}_{t}} k_{s}^{2}+t k_{s s}^{2}+t^{2} k_{s s s}^{2}+\cdots+t^{j}\left|\partial_{s}^{j+1} k\right|^{2} d s+C+C / t^{\theta_{2}}+\cdots+C / t^{\theta_{j}} \\
& \quad+\partial_{t} \sum_{i=1}^{3} t^{2} \mathfrak{q}_{5}\left(\lambda^{i}, k_{s}^{i}\right)+t^{4} \mathfrak{q}_{9}\left(\partial_{t} \lambda^{i}, k_{s s s}^{i}\right)+\cdots+\left.t^{j} \mathfrak{q}_{2 j+1}\left(\partial_{t}^{j / 2-1} \lambda^{i}, \partial_{s}^{j-1} k^{i}\right)\right|_{\text {at the 3-point }} \\
& \leq C+C / t^{\theta} \\
& \quad+\partial_{t} \sum_{i=1}^{3} t^{2} \mathfrak{q}_{5}\left(\lambda^{i}, k_{s}^{i}\right)+t^{4} \mathfrak{q}_{9}\left(\partial_{t} \lambda^{i}, k_{s s s}^{i}\right)+\cdots+\left.t^{j} \mathfrak{q}_{2 j+1}\left(\partial_{t}^{j / 2-1} \lambda^{i}, \partial_{s}^{j-1} k^{i}\right)\right|_{\text {at the 3-point }}
\end{aligned}
$$

for some $\theta<1$. 
Integrating this inequality in time on $[0, t]$ with $t \leq T_{M}$ and taking into account Remark 3.9, we get

$$
\begin{aligned}
\int_{\mathbb{T}_{t}} k^{2}+ & t k_{s}^{2}+\frac{t^{2} k_{s s}^{2}}{2 !}+\cdots+\frac{t^{j}\left|\partial_{s}^{j} k\right|^{2}}{j !} d s \\
\leq & \int_{\mathbb{T}_{0}} k^{2} d s+C T_{M}+C T_{M}^{(1-\theta)}+\sum_{i=1}^{3} t^{2} \mathfrak{q}_{5}\left(\lambda^{i}, k_{s}^{i}\right) \\
& +t^{4} \mathfrak{q}_{9}\left(\partial_{t} \lambda^{i}, k_{s s s}^{i}\right)+\cdots+\left.t^{j} \mathfrak{q}_{2 j+1}\left(\partial_{t}^{j / 2-1} \lambda^{i}, \partial_{s}^{j-1} k^{i}\right)\right|_{\text {at the 3-point }} \\
\leq & \int_{\mathbb{T}_{0}} k^{2} d s+C+t^{2}\left\|\mathfrak{p}_{5}\left(\left|k_{s}\right|\right)\right\|_{L^{\infty}} \\
& +t^{4}\left\|\mathfrak{p}_{9}\left(\left|k_{s s s}\right|\right)\right\|_{L^{\infty}}+\cdots+t^{j}\left\|\mathfrak{p}_{2 j+1}\left(\left|\partial_{s}^{j-1} k\right|\right)\right\|_{L^{\infty}} .
\end{aligned}
$$

Now we absorb all the polynomial terms, after interpolating each one of them between the corresponding "good" integral in the left member and some power of the $L^{2}$ norm of $k$, as we did in showing Proposition 3.13, hence we finally obtain for every even $j \in \mathbb{N}$,

$$
\int_{\mathbb{T}_{t}} k^{2}+t k_{s}^{2}+\frac{t^{2} k_{s s}^{2}}{2 !}+\cdots+\frac{t^{j}\left|\partial_{s}^{j} k\right|^{2}}{j !} d s \leq C_{j}
$$

with $t \in\left[0, T_{M}\right]$ and a constant $C_{j}$ depending only on $\int_{\mathbb{T}_{0}} k^{2} d s$ and the inverses of the lengths of the three curves at time zero.

This family of inequalities clearly implies

$$
\int_{\mathbb{T}_{t}}\left|\partial_{s}^{j} k\right|^{2} d s \leq \frac{C_{j} j !}{t^{j}} \quad \text { for every even } j \in \mathbb{N} \text {. }
$$

Then, passing as before from integral to $L^{\infty}$ estimates by means of inequalities (3.8), we have the following proposition.

PROPOSITION 3.17. For every $\mu>0$ the curvature and all its space derivatives of $\mathbb{T}_{t}$ are uniformly bounded in the time interval $\left[\mu, T_{M}\right]$ (where $T_{M}$ is given by Proposition 3.15) by some constants depending only on $\mu$, the $L^{2}$ norm of $k$ of $\mathbb{T}_{0}$ and the inverses of the lengths of the three curves at time zero.

By means of these a priori estimates we can now work out some results about the flow and improve Theorem 3.1.

THEOREM 3.18. If $[0, T)$ is the maximal time interval of existence of a smooth solution $\mathbb{T}_{t}$ with $T<+\infty$ of Problem (2.1), then

(1) either the inferior limit of the length of at least one curve of $\mathbb{T}_{t}$ goes to zero when $t \rightarrow T$,

(2) or $\varlimsup_{t \rightarrow T} \int_{\mathbb{T}_{t}} k^{2} d s=+\infty$.

Moreover, if the lengths of the three curves are uniformly bounded away from zero, then the superior limit in (2) is a limit and there exists a positive constant $C$ such that $\int_{\mathbb{T}_{t}} k^{2} d s \geq C / \sqrt{T-t} \rightarrow+\infty$ for every $t \in[0, T)$. 
PROOF. If the three lengths are uniformly bounded away from zero and the $L^{2}$ norm of $k$ is bounded, by Proposition 3.14 and Ascoli-Arzelà Theorem, the triods $\mathbb{T}_{t}$ converge in $C^{\infty}$ to a smooth triod $\mathbb{T}_{T}$ as $t \rightarrow T$. Then, applying Theorem 3.1 to $\mathbb{T}_{T}$ we could restart the flow obtaining a smooth evolution on a longer time interval, hence contradicting the maximality of the interval $[0, T)$.

By means of differential inequality (3.10), we have

$$
\frac{d}{d t} \int_{\mathbb{T}_{t}} k^{2} d s \leq C\left(\int_{\mathbb{T}_{t}} k^{2} d s\right)^{3}+C \leq C\left(1+\int_{\mathbb{T}_{t}} k^{2} d s\right)^{3},
$$

which, after integration between $t, r \in[0, T)$ with $t<r$, gives

$$
\frac{1}{\left(1+\int_{\mathbb{T}_{t}} k^{2} d s\right)^{2}}-\frac{1}{\left(1+\int_{\mathbb{T}_{r}} k^{2} d s\right)^{2}} \leq C(r-t) .
$$

Then, if case (1) does not hold, we can choose a sequence of times $r_{j} \rightarrow T$ such that $\int_{\mathbb{T}_{r_{j}}} k^{2} d s \rightarrow+\infty$. Putting $r=r_{j}$ in the inequality above and passing to the limit we get

$$
\frac{1}{\left(1+\int_{\mathbb{T}_{t}} k^{2} d s\right)^{2}} \leq C(T-t)
$$

hence,

$$
\int_{\mathbb{T}_{t}} k^{2} d s \geq \frac{C}{\sqrt{T-t}}-1 \geq \frac{C}{\sqrt{T-t}} \rightarrow+\infty,
$$

for some positive constant $C$.

This theorem obviously implies the following corollary.

COROLlARY 3.19. If $[0, T)$ is the maximal time interval of existence of a smooth solution $\mathbb{T}_{t}$ with $T<+\infty$ and the three lengths are uniformly bounded away from zero, then

$$
\max _{\mathbb{T}_{t}} k^{2} \geq \frac{C}{\sqrt{T-t}} \rightarrow+\infty
$$

as $t \rightarrow T$.

REMARK 3.20. In the case of the evolution $\gamma_{t}$ of a single closed curve in the plane there exist a constant $C>0$ such that if at time $T>0$ a singularity develops, then

for every $t \in[0, T$ ) (see [25]).

$$
\max _{\gamma_{t}} k^{2} \geq \frac{C}{T-t}
$$

If this lower bound on the rate of blowing up of the curvature (which is clearly stronger than the one in inequality (3.13)) holds also in the case of the evolution of a triod is an open problem.

Proposition 3.21. For every $M>0$ there exists a positive time $T_{M}$ such that if the $L^{2}$ norm of the curvature and the inverses of the lengths of the smooth triod $\mathbb{T}_{0}$ are bounded by $M$, then the maximal time of existence $T>0$ of the associated solution of Problem (2.1) with initial data $\mathbb{T}_{0}$ is larger than $T_{M}$. 
Proof. By Proposition 3.15 in the interval $\left[0, \min \left\{T_{M}, T\right\}\right.$ ) the $L^{2}$ norm of $k$ and the inverses of the lengths of the three curves of $\mathbb{T}_{t}$ are bounded by $2 M^{2}+6 M$.

Then, by Theorem 3.18, the value $\min \left\{T_{M}, T\right\}$ cannot coincide with the maximal time of existence, hence $T>T_{M}$.

By means of Proposition 3.17 we can now work out an existence result for an initial triod $\mathbb{T}_{0}$ which is neither smooth nor geometrically smooth, but it is only $C^{2}$ and satisfies the 120 degrees condition.

THEOREM 3.22. If $\mathbb{T}_{0}$ is a $C^{2}$ initial triod (not necessarily geometrically smooth) then there exists a Brakke flow with equality $\mathbb{T}_{t}$ of $\mathbb{T}_{0}$ for some positive time interval $[0, T)$.

Moreover, the triods $\mathbb{T}_{t}$ are geometrically smooth for every time $t>0$ and the curvatures $k^{i}$ belong to $C^{\infty}([0,1] \times(0, T))$, hence the flow is a smooth Brakke flow with equality for every positive time.

Finally, the unit tangents $\tau^{i}$ are continuous in $[0,1] \times[0, T)$ and the function $\int_{\mathbb{T}_{t}} k^{2} d s$ is continuous on $[0, T)$.

Proof. We can approximate in $W^{2,2}(0,1)$ (hence in $\left.C^{1}([0,1])\right)$ the triod $\mathbb{T}_{0}=\cup_{i=1}^{3} \sigma^{i}$ with a family of smooth triods $\mathbb{T}_{j}$, composed of $C^{\infty}$ curves $\sigma_{j}^{i} \rightarrow \sigma^{i}$, as $j \rightarrow \infty$ with $\frac{d \sigma_{j}^{i}(0)}{d x}=\frac{d \sigma^{i}(0)}{d x}$ and $\frac{d^{2} \sigma_{j}^{i}(0)}{d x^{2}}=0$.

By the convergence in $W^{2,2}$ and in $C^{1}$, the inverses of the lengths of the initial curves, the integrals $\int_{\mathbb{T}_{j}} k^{2}+\lambda^{2} d s$ and $\left|\partial_{x} \sigma_{j}^{i}(x)\right|$ (from above and away from zero) for all the approximating triods are equibounded, thus Proposition 3.21 assures the existence of a uniform interval $[0, T)$ of existence of smooth evolutions given by the curves $\gamma_{j}^{i}(x, t):[0,1] \times[0, T) \rightarrow \bar{\Omega}$.

Now, by the same reason, Proposition 3.17 gives uniform estimates on the $L^{\infty}$ norms of the curvature and of all its derivatives in every rectangle $[0,1] \times[\mu, T)$, with $\mu>0$.

We can then select a subsequence (not relabelled) such that the curves $\gamma_{j}^{i}$, after reparametrization proportional to arclength, converge to some $\gamma^{i}(x, t)$ : $[0,1] \times[0, T) \rightarrow \bar{\Omega}\left(\right.$ composing the triods $\left.\mathbb{T}_{t}\right)$,

- uniformly in $[0,1] \times[0, T)$,

- in $C^{\infty}$ in every rectangle $[0,1] \times[\mu, T)$, with $\mu>0$.

Moreover, since all the approximating flows are composed of smooth triods and the curvatures converge smoothly, when $t>0$ the triods $\mathbb{T}_{t}$ are geometrically smooth (see Remark 2.7).

It is then an exercise to see that the unit tangents $\tau^{i}$ are continuous functions also at $t=0$, that is, on all the rectangle $[0,1] \times[0, T)$ (by the uniform control on $\|k\|_{L^{2}}$ and Sobolev embedding theorem). Notice that, the continuity of $\gamma^{i}$ also implies that the measures $\mathcal{H}^{1}\left\llcorner\mathbb{T}_{t}\right.$ weakly ${ }^{\star}$ converge to $\mathcal{H}^{1}\left\llcorner\mathbb{T}_{0}\right.$, where $\mathcal{H}^{1}$ is the Hausdorff one-dimensional measure. 
Now we show that $\mathbb{T}_{t}=\cup_{i=1}^{3} \gamma^{i}$ is a Brakke flow with equality. By the smoothness of the flow for every positive time, we have only to check the derivative $\frac{d}{d t} \int_{\mathbb{T}_{t}} \varphi d s$ at $t=0$.

For every smooth positive test function $\varphi: \bar{\Omega} \times[0, T) \rightarrow \mathbb{R}$ the functional $\int_{\mathbb{T}} \varphi k^{2} d s$ is lower semicontinuous in the convergence of the triods with their unit tangents (see [34], moreover, integrating on [0,t) inequality (3.10) (forgetting the absolute value), for the approximating flows $\gamma_{j}^{i}$, and passing to the limit, we see that the function $\int_{\mathbb{T}_{t}} k^{2} d s$ is actually continuous at $t=0$. Then, by a standard argument, it follows that also the functions $\int_{\mathbb{T}_{t}} \varphi k^{2} d s$ are continuous at $t=0$, for every positive $\varphi$, hence for every smooth function $\varphi$ with compact support in $\Omega$.

Analogously, also the terms $\int_{\mathbb{T}_{t}}\langle\nabla \varphi \mid \underline{k}\rangle d s$ and $\int_{\mathbb{T}_{t}} \varphi_{t} d s$ are continuous at $t=0$, hence integrating equation (2.11), satisfied by the approximating flows, on $[0, t)$ and then passing to the limit we get

$$
\int_{\mathbb{T}_{t}} \varphi d s-\int_{\mathbb{T}_{0}} \varphi d s=-\int_{0}^{t} \int_{\mathbb{T}_{\xi}} \varphi k^{2} d s d \xi+\int_{0}^{t} \int_{\mathbb{T}_{\xi}}\langle\nabla \varphi \mid \underline{k}\rangle d s d \xi+\int_{0}^{t} \int_{\mathbb{T}_{\xi}} \varphi_{t} d s d \xi
$$

which clearly says, by the fundamental theorem of calculus, that the derivative $\frac{d}{d t} \int_{\mathbb{T}_{t}} \varphi d s$ exists at $t=0$ and that $\mathbb{T}_{t}$ is a Brakke flow with equality.

REMARK 3.23.

(1) The relevance of this theorem is that the initial triod is not required to satisfy any compatibility condition, but only to have angles of 120 degrees, in particular, it is not necessary that the sum of the three curvatures at its 3-point is zero.

(2) It should be noticed that if the three initial curves are $C^{\infty}$, the flow $\gamma^{i}$ is smooth till $t=0$ far from the 3 -point, that is, in a closed rectangle included in $[0,1] \times[0, T) \backslash\{(0,0)\}$ we can locally reparametrize the curves to get a smooth flow also at $t=0$.

This follows from the local estimates for the motion by curvature (see [17], for instance).

(3) As we said in the introduction, the next important question is what can be said if the initial triod does not satisfy the 120 degrees condition. One would hope to have a suitable definition of evolution (possibly weak) such that the 120 degrees condition is satisfied instantaneously, that is, at every positive time, like it happens here for the geometrical smoothness.

(4) The uniqueness of the limit $\gamma^{i}$ is an open problem as well as its dependence on the approximating procedure.

Even more important is the geometric uniqueness of such a Brakke flow with continuous unit tangents, forgetting the parametrizations and looking at the triods as subsets of $\mathbb{R}^{2}$.

Finally, if the initial triod is smooth (or geometrically smooth) this flow should be a reparametrization of the smooth evolution given by Theorem 3.1 (see Proposition 3.3). 
(5) We do not know if every Brakke flow with equality starting from a $C^{2}$ initial triod $\mathbb{T}_{0}$, which becomes immediately smooth (possibly requiring also the continuity of the unit tangents), can be obtained in this way, even when the initial triod $\mathbb{T}_{0}$ is smooth.

This problem is clearly related to the uniqueness of the smooth Brakke flows with equality (maybe further restricting the candidates to a special class with extra geometric properties). A positive answer would also allow us to extend to them the analysis of the singularities carried on in the next sections.

REMARK 3.24. We point out that all this section can be extended to networks of curves with many 3-points.

Indeed, an analog of the result of Bronsard and Reitich for such situation (they also remark that) can be obtained generalizing the "algebraic" analysis in their paper [11] in order to show the complementary conditions for the system 3.1 associated to the network. Then, all the estimates can be generalized simply adding the contributions of all the 3-points and of every end point, since each one of them has to satisfy the relations between $k, \lambda$ and their derivatives computed in Section 2 for a single triod.

In all the discussion of this section, we did not take care of the fact that the triods have to remain in the domain $\Omega$ and of the condition of embeddedness, which are required in the formulation of Problem (2.1), actually, we only concentrated on the analytic properties of the solution of the parabolic system (3.1).

It will follow by the geometric results of the next section that since the initial triod is embedded, if the lengths of three curves stay away from zero, then, during the evolution, the triods do not develop self-intersections and "touch" the boundary of $\Omega$ only with their end points.

In the rest of the paper we restrict ourselves only to the smooth flows given by Theorem 3.1 and we will analyse the possible formation of singularities.

\section{4. - Geometric properties of the flow}

Let us consider a smooth evolution $\mathbb{T}_{t}$ in the time interval $[0, T)$ of an initially embedded smooth triod $\mathbb{T}_{0}$ in the convex set $\Omega$.

The first thing we want to show is that the triods cannot get out of $\Omega$.

Proposition 4.1. The triods $\mathbb{T}_{t}$ intersect the boundary of $\Omega$ only at the end points.

Moreover, for every positive time such intersections are transversal.

PRoof. Even if some of the three curves of the initial triod are tangent to $\partial \Omega$ at the end points $P^{i}$, by the strong maximum principle, as $\Omega$ is convex, the intersections become immediately transversal and stay so for every time. 
By continuity, the 3-point cannot hit the boundary at least for some time $T^{\prime}>0$. Then, fixing a time $t \in\left[0, T^{\prime}\right)$, even if the curve $\gamma^{i}(\cdot, t)$ intersects the boundary at some of its inner points, that is, $\gamma^{i}(x, t) \in \partial \Omega$ with $x \neq\{0,1\}$, again by the strong maximum principle and the convexity of $\Omega$ we can conclude that for every subsequent time in the interval $\left(t, T^{\prime}\right)$ there are no more intersections.

This argument clearly implies that if $t_{0}>0$ is the "first time" when the triods intersect the boundary with an inner point, this latter has to be the 3-point $O$. The minimality of $t_{0}$ is then easily contradicted by the convexity of $\Omega$, the 120 degrees condition and the non zero length of the three curves of $\mathbb{T}_{t_{0}}$.

Now we concentrate on the condition of embeddedness.

Given the smooth flow $\mathbb{T}_{t}=F(\mathbb{T}, t)$, we take two points $p=F(x, t)$ and $q=F(y, t)$ belonging to $\mathbb{T}_{t}$ and we define $\Gamma_{p, q}$ to be the geodesic curve contained in $\mathbb{T}_{t}$ connecting $p$ and $q$. Then we let $A_{p, q}$ to be the area of the open region $\mathcal{A}_{p, q}$ in $\mathbb{R}^{2}$ enclosed by the segment $[p, q]$ and the curve $\Gamma_{p, q}$. When the region $\mathcal{A}_{p, q}$ is not connected, we let $A_{p, q}$ to be the sum of the areas of its connected components.

We consider the function $\Phi_{t}: \mathbb{T} \times \mathbb{T} \rightarrow \mathbb{R} \cup\{+\infty\}$ as

$$
\Phi_{t}(x, y)= \begin{cases}\frac{|p-q|^{2}}{A_{p, q}} & \text { if } x \neq y, \\ 4 \sqrt{3} & \text { if } x \text { and } y \text { coincide with the 3-point } O \text { of } \mathbb{T}, \\ +\infty & \text { if } x=y \neq O\end{cases}
$$

where $p=F(x, t)$ and $q=F(y, t)$.

Since $\mathbb{T}_{t}$ is smooth and the 120 degrees condition holds, it is easy to check that $\Phi_{t}$ is a lower semicontinuous function. Hence, by the compactness of $\mathbb{T}$, the following infimum is actually a minimum

$$
E(t)=\inf _{x, y \in \mathbb{T}} \Phi_{t}(x, y)
$$

for every $t \in[0, T)$.

Similar geometric quantities have already been applied in [23], [13] and [26].

If the triod $\mathbb{T}_{t}$ has no self-intersections we have $E(t)>0$, the converse is clearly also true.

Moreover, $E(t) \leq \Phi_{t}(0,0)=4 \sqrt{3}$ always holds, thus when $E(t)>0$ the two points $(p, q)$ of a minimizing pair $(x, y)$ can coincide if and only if $p=q=O$.

Finally, since the evolution is smooth it is easy to see that the function $E:[0, T) \rightarrow \mathbb{R}$ is continuous.

These properties set the question of the possible self-intersections of solutions of Problem (2.1) that we let open in the previous section at the end of the proof of Theorem 3.1 (the fact that the triods remain in $\Omega$ is shown by Proposition 4.1). Indeed, if the initial triod $\mathbb{T}_{0}$ is embedded, we have $E(0)>0$, hence $E(t)>0$ for some time. 
Now we want to show something more, that is, in all the maximal interval of existence of a smooth flow self-intersections cannot happen, in other words, if $E(0)>0$ then $E(t)>0$ for every $t \in[0, T)$.

Since we are dealing with embedded triods, with a little abuse of notation, we consider the function $\Phi_{t}$ defined on $\mathbb{T}_{t} \times \mathbb{T}_{t}$ and we speak of a minimizing pair for the couple of points $(p, q) \in \mathbb{T}_{t} \times \mathbb{T}_{t}$ instead of $(x, y) \in \mathbb{T} \times \mathbb{T}$.

LEMMA 4.2. Assume that $0<E(t)<4 \sqrt{3}$, then for any minimizing pair $(p, q)$ we have $p \neq q$ and neither $p$ nor $q$ coincides with the 3-point $O$ of $\mathbb{T}_{t}$.

Moreover, it is always possible to find a minimizing pair such that $\mathcal{A}_{p, q}$ is a single connected region and the segment $[p, q]$ meet the (one or two) curves containing $p$ and $q$ only at these points and with transversal intersection.

Proof. We already saw that by the very definition of $\Phi_{t}$, the inequality $0<E(t)<4 \sqrt{3}$ implies $p \neq q$. The proof that $p$ and $q$ can be chosen in order that the region $\mathcal{A}_{p, q}$ is connected and the segment $[p, q]$ does not intersect the curves which contain the two points, goes like in [13, Lemma 2.1]).

We prove the first claim, assuming by contradiction that $p=O \in \mathbb{T}_{t}$ and $\Phi_{t}(O, q)=E(t)$. By the above, we can suppose that the segment $[O, q]$ is contained in in the sector between the curves $\gamma^{1}$ and $\gamma^{2}$, that $q \in \gamma^{1}$ and that the region $\mathcal{A}_{O, q}$ is bounded by such segment and the curve $\gamma^{1}$.

If the angle $\alpha \geq 0$ formed by $[O, q]$ and $\tau^{2}(O, t)$ is smaller than 90 degrees it is easy to see that moving a little the point $p$ along $\gamma^{2}$, the distance $|p-q|$ decreases while the area $A_{p, q}$ increases, hence the ratio $|O-q|^{2} / A_{O, q}$ cannot be minimal.

Thus the width of the angle $\alpha$ has to be greater or equal than 90 degrees.

We consider then the points $p(s)=\gamma^{1}(x(s), t)$ with arclength parameter $s \in[0, \varepsilon)$ (then $p(0)=O$ and $\frac{d p(0)}{d s}=\tau^{1}(O, t)$ ) and we compute the right derivative at $s=0$ of $\Phi_{t}(p(s), q)$ (see the proof of Proposition 4.4),

$$
\begin{aligned}
\left.\frac{d}{d s} \Phi_{t}(p(s), q)\right|_{s=0} & =\left.\frac{A_{p(s), q} \frac{d}{d s}|p(s)-q|^{2}-|p(s)-q|^{2} \frac{d}{d s} A_{p(s), q}}{A_{p(s), q}^{2}}\right|_{s=0} \\
& =\frac{-2 A_{p(0), q}|p(0)-q| \cos (120-\alpha)+1 / 2|p(0)-q|^{3} \sin (120-\alpha)}{A_{p(0), q}^{2}} \\
& =\frac{|O-q|}{2 A_{O, q}^{2}}\left[-4 A_{O, q} \cos (120-\alpha)+|O-q|^{2} \sin (120-\alpha)\right]
\end{aligned}
$$

which has to be non negative by minimality.

Hence, it follows that $4 A_{O, q} \cos (120-\alpha) \leq|O-q|^{2} \sin (120-\alpha)$ and

$$
4 \cot (120-\alpha) \leq \frac{|O-q|^{2}}{A_{O, q}}=\Phi_{t}(O, q)=E(t) .
$$

Since $\pi / 2 \leq \alpha \leq 2 \pi / 3$ we have $\cot (120-\alpha) \geq \sqrt{3}$, hence we conclude $E(t) \geq 4 \sqrt{3}$ which is in contradiction with the initial hypothesis. 
Finally, as neither $p$ nor $q$ coincides with the 3-point, if $[p, q]$ intersects $\mathbb{T}_{t}$ tangentially at $p$ or $q$, moving such point a little in the direction which decreases the distance $|p-q|$, the area $A_{p, q}$ is little changed, so a variation as above gives a contradiction (see [13, Lemma 2.1]).

REMARK 4.3. Looking at the proof of the connectedness in [13], it can be proved also that if there is at least one minimizing pair such that both its points are distinct from the end points $P^{i}$, then the same holds also for the pair $(p, q)$ with $\mathcal{A}_{p, q}$ connected whose existence is assured by this lemma.

PROPOSITION 4.4. The function $E(t)$ is monotone increasing in every time interval where $0<E(t)<4 \sqrt{3}$ and for at least one minimizing pair $(p, q)$ of $\Phi_{t}$ neither $p$ nor $q$ coincides with one of the end points $P^{i}$.

Proof. We assume that $0<E(t)<4 \sqrt{3}$ and that there exists a minimizing pair $(p, q)$ for $\Phi_{t}$ such that the two points are both distinct from the end points $P^{i}$, for every $t$ in some interval of time. Since $E(t)$ is a locally Lipschitz function, to prove the statement it is then enough to show that $\frac{d E(t)}{d t}>0$ for every time $t$ such that this derivative exists (which happens almost everywhere in the interval).

Fixed a minimizing pair $(p, q)$ at time $t$, satisfying the conclusions of Lemma 4.2 and Remark 4.3, we choose a value $\varepsilon>0$ smaller than the geodesic distances of $p$ and $q$ from the 3-point $O$ of $\mathbb{T}_{t}$ and between them, moreover if $p$ and $q$ both belong to the same curve we can also suppose that $q$ is the closest to $O$.

By simplicity, we discuss the situation where the points $p, q$ are like in Figure 1, the computations in the other cases are analogous.

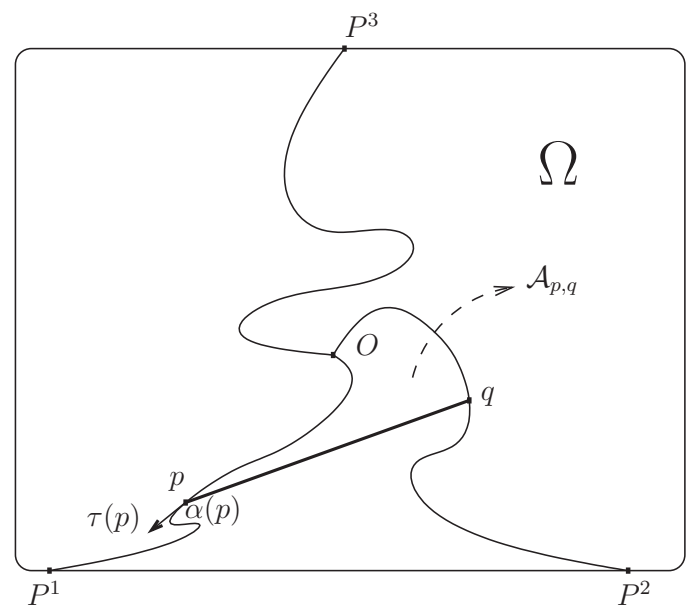

Fig. 1. 
Possibly taking a smaller $\varepsilon>0$, we fix an arclength coordinate $s \in(-\varepsilon, \varepsilon)$ and a local parametrization $p(s)$ of the curve containing in a neighborhood of $p=p(0)$, with the same orientation of the original one. Let $\eta(s)=|p(s)-q|$ and $A(s)=A_{p(s), q}$, since

$$
E(t)=\min _{s \in(-\varepsilon, \varepsilon)} \frac{\eta^{2}(s)}{A(s)}=\frac{\eta^{2}(0)}{A(0)}
$$

if we differentiate in $s$ we obtain

$$
\frac{d \eta^{2}(0)}{d s} A(0)=\frac{d A(0)}{d s} \eta^{2}(0)
$$

As the intersection of the segment $[p, q]$ with the triod is transversal, we have an angle $\alpha(p) \in(0, \pi)$ determined by the unit tangent $\tau(p)$ and the vector $q-p$. We compute

$$
\begin{aligned}
& \frac{d \eta^{2}(0)}{d s}=-2\langle\tau(p) \mid q-p\rangle=-2|p-q| \cos \alpha(p) \\
& \frac{d A(0)}{d s}=\frac{1}{2}|\tau(p) \wedge(q-p)|=\frac{1}{2}\langle\nu(p) \mid q-p\rangle=\frac{1}{2}|p-q| \sin \alpha(p)
\end{aligned}
$$

Putting these derivatives in equation (4.2) and recalling that $\eta^{2}(0) / A(0)=E(t)$, we get

$$
\cot \alpha(p)=-\frac{|p-q|^{2}}{4 A_{p, q}}=-\frac{E(t)}{4} .
$$

Since $0<E(t)<4 \sqrt{3}$ we get $-\sqrt{3}<\cot \alpha(p)<0$ which implies

$$
\frac{\pi}{2}<\alpha(p)<\frac{5}{6} \pi
$$

The same argument clearly holds for the point $q$, hence defining $\alpha(q) \in(0, \pi)$ to be the angle determined by the unit tangent $\tau(q)$ and the vector $p-q$, by equation (4.3) it follows that $\alpha(p)=\alpha(q)$ and we simply write $\alpha$ for both.

We consider now a different variation, moving at the same time the points $p$ and $q$, in a way that $\frac{d p(s)}{d s}=\tau(p(s))$ and $\frac{d q(s)}{d s}=\tau(q(s))$. we have

As above, letting $\eta(s)=|p(s)-q(s)|$ and $A(s)=A_{p(s), q(s)}$, by minimality

$$
\frac{d \eta^{2}(0)}{d s} A(0)=\frac{d A(0)}{d s} \eta^{2}(0) \quad \text { and } \quad \frac{d^{2} \eta^{2}(0)}{d s^{2}} A(0) \geq \frac{d^{2} A(0)}{d s^{2}} \eta^{2}(0)
$$


Computing as before,

$$
\begin{aligned}
\frac{d \eta^{2}(0)}{d s}= & 2\langle p-q \mid \tau(p)-\tau(q)\rangle \\
\frac{d A(0)}{d s}= & -4|p-q| \cos \alpha=-\frac{1}{2}\langle p-q \mid v(p)+v(q)\rangle \\
\frac{d^{2} \eta^{2}(0)}{d s^{2}}= & |p-q| \sin \alpha=2\langle\tau(p)-\tau(q) \mid \tau(p)-\tau(q)\rangle \\
& +2\langle p-q \mid k(p) v(p)-k(q) v(q)\rangle \\
= & 2|\tau(p)-\tau(q)|^{2}+2\langle p-q \mid k(p) v(p)-k(q) v(q)\rangle \\
= & 8 \cos ^{2} \alpha+2\langle p-q \mid k(p) v(p)-k(q) v(q)\rangle \\
\frac{d^{2} A(0)}{d s^{2}}= & -\frac{1}{2}\langle\tau(p)-\tau(q) \mid v(p)+v(q)\rangle+\frac{1}{2}\langle p-q \mid k(p) \tau(p)+k(q) \tau(q)\rangle \\
= & -\frac{1}{2}\langle\tau(p) \mid v(q)\rangle+\frac{1}{2}\langle\tau(q) \mid v(p)\rangle+\frac{1}{2}\langle p-q \mid k(p) \tau(p)+k(q) \tau(q)\rangle \\
= & -2 \sin \alpha \cos \alpha-1 / 2|p-q|(k(p)-k(q)) \cos \alpha .
\end{aligned}
$$

Putting the last two relations in the second inequality of (4.4), we get

$$
\begin{aligned}
\left(8 \cos ^{2} \alpha\right. & +2\langle p-q \mid k(p) v(p)-k(q) v(q)\rangle) A_{p, q} \\
& \geq(-2 \sin \alpha \cos \alpha-1 / 2|p-q|(k(p)-k(q)) \cos \alpha)|p-q|^{2}
\end{aligned}
$$

hence, keeping in mind that $\tan \alpha=-4 / E(t)$, by equation (4.3), we obtain

$$
\begin{aligned}
2 A_{p, q}\langle p-q| & k(p) v(p)-k(q) v(q)\rangle+1 / 2|p-q|^{3}(k(p)-k(q)) \cos \alpha \\
& \geq-2 \sin \alpha \cos \alpha|p-q|^{2}-8 A_{p, q} \cos ^{2} \alpha \\
& =-2 A_{p, q} \cos ^{2} \alpha\left(\tan \alpha \frac{|p-q|^{2}}{A_{p, q}}+4\right) \\
& =-2 A_{p, q} \cos ^{2} \alpha\left(-\frac{4}{E(t)} E(t)+4\right)=0 .
\end{aligned}
$$

We consider now a time $t_{0}$ such that the derivative $\frac{d E\left(t_{0}\right)}{d t}$ exists and we compute it with the following standard trick,

$$
\frac{d E\left(t_{0}\right)}{d t}=\left.\frac{\partial}{\partial t} \Phi_{t}(p, q)\right|_{t=t_{0}}
$$

for any pair $(p, q)$ such that $p, q \in \mathbb{T}_{t_{0}}$ and $\frac{|p-q|^{2}}{A_{p, q}}=E\left(t_{0}\right)$. 
Considering then a minimizing pair $(p, q)$ for $\Phi_{t_{0}}$ with all the previous properties, by minimality, we are free to choose the "motion" of the points $p(t), q(t)$ "inside" the triods $\mathbb{T}_{t}$ in computing such partial derivative.

Since locally the triods are moving by curvature and we know that neither $p$ nor $q$ coincides with the 3-point or the end points, we can find $\varepsilon>0$ and two smooth curves $p(t), q(t) \in \mathbb{T}_{t}$ for every $t \in\left(t_{0}-\varepsilon, t_{0}+\varepsilon\right)$ such that

$$
\begin{array}{lll}
p\left(t_{0}\right)=p & \text { and } & \frac{d p(t)}{d t}=k(p(t), t) v(p(t), t), \\
q\left(t_{0}\right)=q & \text { and } & \frac{d q(t)}{d t}=k(q(t), t) v(q(t), t) .
\end{array}
$$

Then,

$$
\begin{aligned}
\frac{d E\left(t_{0}\right)}{d t} & =\left.\frac{\partial}{\partial t} \Phi_{t}(p, q)\right|_{t=t_{0}} \\
& =\left.\frac{1}{A_{p, q}^{2}}\left(A_{p, q} \frac{d|p(t)-q(t)|^{2}}{d t}-|p-q|^{2} \frac{d A_{p(t), q(t)}}{d t}\right)\right|_{t=t_{0}}
\end{aligned}
$$

With a straightforward computation we get the following equalities,

$$
\begin{aligned}
\left.\frac{d|p(t)-q(t)|^{2}}{d t}\right|_{t=t_{0}} & =2\langle p-q \mid k(p) v(p)-k(q) v(q)\rangle \\
\left.\frac{d A_{p(t), q(t)}}{d t}\right|_{t=t_{0}} & =\int_{\Gamma_{p, q}}\left\langle\underline{k}(s) \mid v_{\Gamma_{p, q}}\right\rangle d s+1 / 2|p-q|\left\langle v_{[p, q]} \mid k(p) v(p)+k(q) v(q)\right\rangle \\
& =2 \alpha-5 \pi / 3-1 / 2|p-q|(k(p)-k(q)) \cos \alpha
\end{aligned}
$$

where we wrote $v_{\Gamma p, q}$ and $v_{[p, q]}$ for the exterior unit normal to the region $\mathcal{A}_{p, q}$, respectively at the points of the geodesic $\Gamma_{p, q}$ and of the segment $[p, q]$.

Substituting these derivatives in equation (4.6) we get

$$
\begin{aligned}
& \frac{d E\left(t_{0}\right)}{d t} \\
& =\frac{1}{A_{p, q}^{2}}\left(2 A_{p, q}\langle p-q \mid k(p) v(p)-k(q) v(q)\rangle+1 / 2 \cos \alpha|p-q|^{3}(k(p)-k(q))\right) \\
& \quad-\frac{|p-q|^{2}}{A_{p, q}^{2}}\left(2 \alpha-\frac{5 \pi}{3}\right),
\end{aligned}
$$

and, by equation (4.5), the first term in parentheses is non negative, hence

$$
\frac{d E\left(t_{0}\right)}{d t} \geq-\frac{|p-q|^{2}}{A_{p, q}^{2}}\left(2 \alpha-\frac{5 \pi}{3}\right) .
$$


By equation (4.3) we have $\alpha=\arctan \left(-4 / E\left(t_{0}\right)\right)$, hence the following inequality holds

$$
\frac{d E\left(t_{0}\right)}{d t} \geq \frac{2 E\left(t_{0}\right)}{A_{p, q}}\left(\arctan \left(4 / E\left(t_{0}\right)\right)-\arctan (1 / \sqrt{3})\right)>0 .
$$

As the area $A_{p, q}$ is bounded by the area of $\Omega$, we conclude that for every $t$ in an interval such that the minimum of $\Phi_{t}$ is taken by at least one pair of inner points and the derivative of $E(t)$ exists,

$$
\frac{d E\left(t_{0}\right)}{d t} \geq 2 C E\left(t_{0}\right)\left(\arctan \left(4 / E\left(t_{0}\right)\right)-\arctan (1 / \sqrt{3})\right)
$$

for the time independent constant $C=1 / \operatorname{Area}(\Omega)>0$.

REMARK 4.5. All this analysis can be extended step by step to a network with many 3-points but without loops, that is, a tree. The presence of loops complicates the analysis of the minimality properties, because of the possible presence of more than one geodesic in $\mathbb{T}_{t}$ between the two points $p$ and $q$.

We now show that on all $[0, T)$ we have $E(t)>0$.

THEOREM 4.6. If $\Omega$ is bounded and strictly convex, there exists a constant $C>0$ depending only on $\mathbb{T}_{0}$ such that $E(t)>C>0$ for every $t \in[0, T)$.

Hence, the triods $\mathbb{T}_{t}$ remain embedded in all the maximal interval of existence of the flow.

Proof. We define three flows of networks of curves $\mathbb{H}_{t}^{1}, \mathbb{H}_{t}^{2}, \mathbb{H}_{t}^{3}$ in the interval $[0, T)$. The network $\mathbb{H}_{t}^{i}$ is obtained as the set theoretic union of $\mathbb{T}_{t}$ with its symmetric image $\mathbb{T}_{t}^{i}$ with respect to the point $P^{i}$.

As the triods $\mathbb{T}_{t}$ are contained in the convex $\Omega$ which is strictly convex, this operation does not introduces self-intersections and since, by Lemma 3.10 all the even derivatives of $k$ and $\lambda$ are zero at the end points $P^{i}$, each one of $\mathbb{H}_{t}^{1}, \mathbb{H}_{t}^{2}, \mathbb{H}_{t}^{3}$ is a smooth flow by curvature of a centrally symmetric network, which is a tree and it is composed of five curves, two 3-points and four fixed end points.

We define for these networks the functions $E^{1}, E^{2}, E^{3}:[0, T) \rightarrow \mathbb{R}$, analogous to the function $E:[0, T) \rightarrow \mathbb{R}$ of $\mathbb{T}_{t}$ and we set $\Pi(t)=\min \left\{E^{1}(t), E^{2}(t), E^{3}(t)\right\}$ which clearly turns out to be a locally Lipschitz function on $[0, T)$ satisfying $\Pi(t) \leq E^{i}(t) \leq E(t)$ for every time $t$ and index $i \in\{1,2,3\}$, since every $\mathbb{H}_{t}^{i}$ contains a copy of $\mathbb{T}_{t}$ (actually two copies). Moreover, as there are no self-intersections by construction, $\Pi(0)>0$.

Showing that for every time $\Pi(t)>0$, we prove the theorem.

We consider a time $t \in[0, T)$ such that the time derivatives of $\Pi$ and of all the $E^{i}$ exist (almost everywhere), then for every index $i \in\{1,2,3\}$ such that $E^{i}(t)=\Pi(t)$ we must have $\frac{d E^{i}(t)}{d t}=\frac{d \Pi(t)}{d t}$.

Extending the previous analysis to the flow $\mathbb{H}_{t}^{i}$, which is a tree hence Remark 4.5 applies, if the minimum $E^{i}(t)=\Pi(t)$ is taken by at least one 
pair of inner points (notice that $P^{i}$ became an inner point for $\mathbb{H}_{t}^{i}$ ), then this derivative is positive.

If every minimizing pair $(p, q)$ is constituted by two end points then, by our construction, the squared distance $|p-q|^{2}$ is bounded from below and $E^{i}(t)=\Pi(t)>C>0$ for some uniform constant $C>0$ independent of $t \in[0, T)$, determined by $\mathbb{T}_{0}$.

The same holds if one is an end point of $\mathbb{T}_{t}$, different from $P^{i}$, and the other is a point of $\mathbb{T}_{t}^{i}$ and viceversa ( $\mathbb{H}_{t}^{i}$ is centrally symmetric).

In the last case, when $(p, q)$ is composed of an inner point and an end point of $\mathbb{H}_{t}^{i}$, both in the same copy of $\mathbb{T}_{t}$, if we consider the same pair and associated region, contained in the other two networks, in at least one of them the two points are both inner, for instance this happens in the network, say $\mathbb{H}_{t}^{j}$, if the end point of $\mathbb{H}_{t}^{i}$ in the pair is a copy of $P^{j}$. Hence, we found an inner minimizing pair of $\mathbb{H}_{t}^{j}$, that is $E^{j}(t)=E^{i}(t)=\Pi(t)$, which implies, by the previous discussion, that $\frac{d E^{i}(t)}{d t}>0$ so $\frac{d \Pi(t)}{d t}>0$.

We conclude that if $\Pi(t)$ is under some constant $C>0$ on $[0, T)$ then it is increasing. Since $\Pi(0)>0$ this argument gives a uniform bound from below on $\Pi(t)$ on $[0, T)$, hence on $E(t)$.

REMARK 4.7. The reason why we put the strict convexity of $\Omega$ in the hypothesis is that, in the very special situation such that the three end points $P^{i}$ stay on a line and one of them is the middle point of the segment determined by the other two, then the symmetry operation with respect to this middle point produces an intersection between $\mathbb{T}_{t}$ and its image at the other two end points, hence the argument above cannot be applied since two loops have formed.

All the results of this section, in particular the previous one can be generalized to networks of curves with many 3-points which are trees, that is, without loops in a strictly convex set $\Omega$.

\section{5. - Blow up and self-similar solutions}

As before we suppose to have a smooth embedded solution $\mathbb{T}_{t}$ of Problem (2.1) in a bounded and strictly convex $\Omega \subset \mathbb{R}^{2}$ on a maximal time interval $[0, T)$. Moreover, we assume that there is a constant $\delta>0$ which uniformly bounds from below the lengths $L^{i}(t)$ of the three curves of $\mathbb{T}_{t}$.

By Theorem 3.18 and Corollary 3.19 the maximum of the modulus of the curvature and its $L^{2}$ norm go to $+\infty$, as $t \rightarrow T$.

As it is standard, we divide the possible singularities in two cases (recall Remark 3.20) according to the rate of blow up of the curvature.

We say that we have a Type I singularity (or a fast singularity) if there exists a constant $C$ such that

$$
\max _{\mathbb{T}_{t}} k^{2} \leq \frac{C}{T-t}
$$

for every $t \in[0, T)$. 
In the case inequality (5.1) does not hold for any constant $C$ we say that the singularity is of Type II (or a slow singularity).

Blowing up in a proper way the evolving triods around a Type I singularity, we obtain as possible limits an unbounded and embedded triod without end points or an unbounded and embedded curve with at most one end point, moving by curvature simply shrinking homothetically.

In the standard case of the evolution of a closed curve in the plane, it is possible to do a blow up of a Type II singularity obtaining a translating solution of the motion by curvature which has to be a straight line or the grim reaper (see [5], [22]). In our situation dealing with triods, we are not able at the moment to get the same conclusion, but we can anyway hope to exclude the presence of Type II singularities, analysing the possible blow up.

In the rest of this section we classify the embedded triods without end points (see Remark 1.4) shrinking homothetically by curvature and, since they could become relevant, we classify also the translating ones.

DeFinition 5.1. We say that a family of unbounded triods without end points is a smooth flow by curvature in $\mathbb{R}^{2}$ if locally, in space and time, it can be parametrized by some maps giving a smooth flow as in Definition 2.4 (but with the end points free to move).

\section{1. - Classification of homothetic flows of triods}

We can clearly suppose that the origin of $\mathbb{R}^{2}$ is the center of homothety.

By a straightforward computation, it can be seen that the curves $\gamma$ in $\mathbb{R}^{2}$ which are homothetically shrinking around the origin of $\mathbb{R}^{2}$ under the curvature flow satisfy the equation $k=-\lambda\langle x \mid \nu\rangle$, for some positive constant $\lambda$, at every point $x \in \gamma$.

Then, by a rescaling, we can suppose that $k=-\langle x \mid \nu\rangle$, that is $\lambda=1$.

LEMMA 5.2. If a closed, unbounded and embedded triod $\mathbb{T}$ in $\mathbb{R}^{2}$ without end points satisfies $k=-\langle x \mid \nu\rangle$ at every point $x \in \mathbb{T}$, then the 3-point coincides with the origin of $\mathbb{R}^{2}$ and the three curves of the triod are halfines forming angles of 120 degrees.

Proof. By the work of Abresch and Langer [1], since the three curves of the triod satisfy the equation above, they can be only halflines, pieces of circles or pieces of a special family of curves (curves of Abresch and Langer) described in [1], which are bounded, periodic and with transversal self-intersections. Since the triod has no end points, if an edge is a piece of one of these curves or of a circle, following this edge in the direction opposite to the 3-point one would get a self-intersection which is not present, hence, these two possibilities have to be excluded.

Then, the three curves are halflines meeting with angles of 120 degrees and, by means of the equation above, it follows immediately that the 3-point has to coincide with the origin of $\mathbb{R}^{2}$. 
By the same argument used in this proof we can also get the following two lemmas that we will need in the next section.

LEMMA 5.3. If a closed, unbounded and embedded curve $\gamma$ without end points satisfies $k=-\langle x \mid v\rangle$ at every point $x \in \gamma$, then the curve is a straight line through the origin of $\mathbb{R}^{2}$.

LEMMA 5.4. If a closed, unbounded and embedded curve $\gamma$ with only one end satisfies $k=-\langle x \mid v\rangle$ at every point $x \in \gamma$, then the curve is a halfline.

These lemmas implies the following classification result.

Proposition 5.5. If $\mathbb{T}_{t}$ is a flow of unbounded triods (curves) without end points (with one or without end points) shrinking homothetically during the motion by curvature, then $\mathbb{T}_{t}$ is composed of three halfines forming three angles of 120 degrees (one halfine or a straight line), hence it is not moving at all $(k \equiv 0)$.

\section{2. - Classification of translating flows of triods}

The curves $\gamma$ in $\mathbb{R}^{2}$ which move by translation with constant velocity $w \in \mathbb{R}^{2}$, for the curvature flow satisfy the equation $k=\langle w \mid v\rangle$ at every point (observe that this equation is translation invariant).

Definition 5.6. The grim reaper relative to the vector $e_{1}$ is the graph of the function $x=-\log (\cos y)$ in $\mathbb{R}^{2}$ when $y$ varies in the interval $(-\pi / 2, \pi / 2)$.

The grim reaper relative to a non zero vector $w \in \mathbb{R}^{2}$ is obtained rotating and dilating the grim reaper relative to $e_{1}$, in a way to make this latter coincide with $w$.

REMARK 5.7. Notice that the grim reaper relative to $w$ is a smooth convex curve asymptotic to two straight lines in $\mathbb{R}^{2}$ parallel to such vector.

LEMMA 5.8. If a closed, unbounded and embedded triod $\mathbb{T}$ in $\mathbb{R}^{2}$ without end points satisfies $k=\langle w \mid v\rangle$ at every point, with $w \neq 0$, then its curves are halflines parallel to $w$ or translated copies of pieces of the grim reaper relative to $w$ (see Figure 2), meeting at the 3-point with angles of 120 degrees (it clearly follows that at most one curve is a halfline).

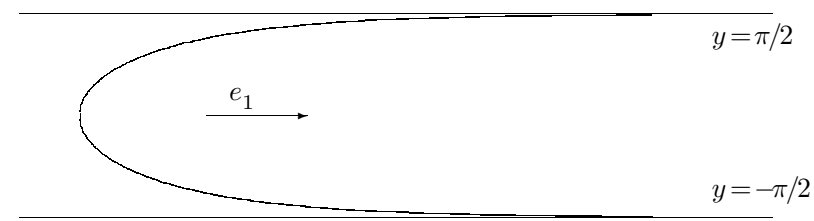

Fig. 2. The grim reaper relative to $e_{1}$. 

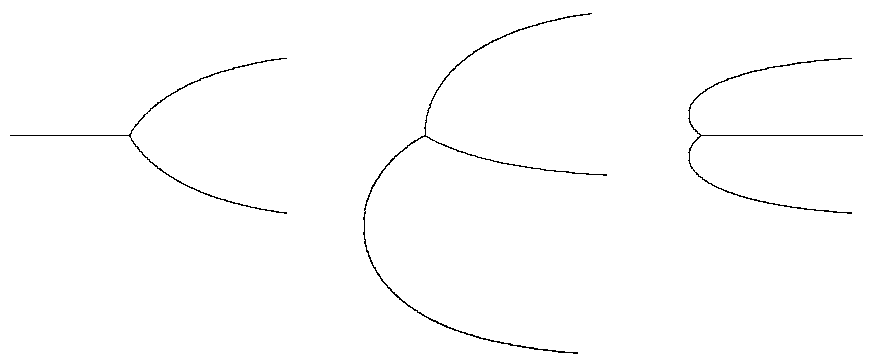

Fig. 3. Some examples of translating triods.

Proof. At every inner point of the curves composing the triod, differentiating in arclength the equation above we get $k_{s}=-k\langle w \mid \tau\rangle$, hence, if the curvature is zero at some point, it has to be zero on all the curve, so this latter is a halfline parallel to $w$.

If $k$ is non zero, then differentiating again we obtain $k_{s s}=-k_{s}\langle w \mid \tau\rangle-$ $k^{2}\langle w \mid v\rangle=k_{s}^{2} / k-k^{3}$ which is the equation of a grim reaper. Integrating such equation we get the thesis.

With the same arguments we can also prove the following lemmas.

LEMMA 5.9. If a closed, unbounded and embedded curve $\gamma$ in $\mathbb{R}^{2}$ without end points satisfies $k=\langle w \mid v\rangle$ at every point, then it is either a straight line parallel to the vector $w$ or a translated copy of the grim reaper relative to $w$.

LEMMA 5.10. If a closed, unbounded and embedded curve $\gamma$ with only one end satisfies $k=\langle w \mid v\rangle$ at every point, then it is either a halfline parallel to the vector $w$ or a translated copy of a piece of the grim reaper relative to $w$.

Hence, we conclude as above.

Proposition 5.11. If $\mathbb{T}_{t}$ is a flow of unbounded triods (curves) without end points (without or with only one end point) translating during the motion by curvature, then every $\mathbb{T}_{t}$ is one of the sets of Lemma 5.8 (5.9 or 5.10).

\section{6. - Type I singularities}

In this section we study the Type I singularities of a smooth flow of triods $\mathbb{T}_{t}$, described by a map $F: \mathbb{T} \times[0, T) \rightarrow \bar{\Omega}$ (see after Definition 2.5), in a bounded and strictly convex $\Omega \subset \mathbb{R}^{2}$, on a maximal time interval $[0, T)$.

We remark that in this section, we will often consider the triods as subsets of $\mathbb{R}^{2}$, without mentioning $\Omega$.

By the Type $I$ assumption, there exists a constant $C>0$ such that

$$
\lim _{t \rightarrow T} \max _{\mathbb{T}_{t}} k^{2}=+\infty \quad \text { and } \quad k^{2}(p, t) \leq \frac{C}{T-t}
$$

for every $p \in \mathbb{T}$ and $t \in[0, T)$. 
LEMMA 6.1. One of the following three (mutually non exclusive) possibilities holds:

(1) There is an index $i \in\{1,2,3\}$ and a sequence of times $t_{j} \nearrow T$ such that

$$
\left[k^{i}\left(O, t_{j}\right)\right]^{2} \geq \frac{C}{T-t_{j}}
$$

for some positive constant $C$.

(2) We have the estimate

$$
\max _{\mathbb{T}_{t}} k^{2}=\frac{o(1)}{T-t}
$$

as $t \rightarrow T$.

(3) The maximum of $k^{2}$ is definitely not taken at the point $O$ and

$$
\max _{\mathbb{T}_{t}} k^{2} \geq \frac{1}{2(T-t)}
$$

for every $t$ larger than some $t_{0} \in[0, T)$.

Proof. We consider the non negative, locally Lipschitz functions $f(t)=$ $\max _{\mathbb{T}_{t}} k^{2}$ and $g(t)=(T-t) \max _{\mathbb{T}_{t}} k^{2}$.

If we are not in the first case, $\lim _{t \rightarrow T}(T-t)\left[k^{i}(O, t)\right]^{2}=0$ for every index $i \in\{1,2,3\}$. Setting $g_{O}(t)=\max _{i \in\{1,2,3\}}(T-t)\left[k^{i}(O, t)\right]^{2}$, if $g(t)>g_{O}(t)$ then the maximum of $k^{2}$ at time $t$ is taken in the interior of one of the three curves ( $k$ is zero at the three end points).

If $g>g_{O}$ does not hold definitely, there exists a time $t_{0}$ such that $g_{O}(t)<$ $1 / 4$ for every $t>t_{0}$ and another time $t_{1}>t_{0}$ such that $g\left(t_{1}\right)=g_{O}\left(t_{1}\right)<1 / 4$.

Then, following Huisken [25], at every time $t$ such that $g(t)>g_{O}(t)$ by the parabolic maximum principle the function $f(t)=\max _{\mathbb{T}_{t}} k^{2}$ satisfies the differential inequality

$$
\frac{d}{d t} f(t) \leq 2 \max _{\mathbb{T}_{t}} k^{4}=2 f^{2}(t)
$$

and the function $g(t)$,

$$
\frac{d}{d t} g(t) \leq 2(T-t) \max _{\mathbb{T}_{t}} k^{4}-\max _{\mathbb{T}_{t}} k^{2}=(2 g-1) f(t) .
$$

If $J$ is the set of times $t \in\left[t_{1}, T\right)$ such that $g(t)=g_{O}(t)<1 / 4$, then in $\left[t_{1}, T\right) \backslash J$, which is a union of open intervals, the function $g$ is decreasing, by a simple ODE's argument. It follows that $g(t) \leq g_{O}(\sup (J \cap[0, t]))$ and since we supposed that $\lim _{t \rightarrow T} \sup (J \cap[0, t])=T$, we conclude $\lim _{t \rightarrow T} g(t)=0$. This means that we are in the second case.

If instead $g>g_{O}$ definitely, then after some time the maximum of $k^{2}$ is always taken at some inner points, hence inequality (6.2) holds and since $f$ goes to $+\infty$ as $t \rightarrow T$, by integration we get the bound from below (Huisken [25])

$$
\max _{\mathbb{T}_{t}} k^{2} \geq \frac{1}{2(T-t)}
$$

for every $t$ larger than some $t_{0}$, that is, we are in the third case. 
In the second situation above we can get something more.

LEMMA 6.2. If the curvature of the triods satisfies

$$
\max _{\mathbb{T}_{t}} k^{2}=\frac{o(1)}{T-t} .
$$

then there exists an index $i \in\{1,2,3\}$ and a sequence of times $t_{j} \nearrow T$ such that

$$
k^{2}(p, t) \leq\left[k^{i}\left(O, t_{j}\right)\right]^{2} \text { for every } t \leq t_{j}, p \in \mathbb{T} \text { and }\left[k^{i}\left(O, t_{j}\right)\right]^{2} \nearrow+\infty .
$$

Proof. We start studying the non decreasing hull $\tilde{f}(t)=\sup _{\xi \in[0, t]} f(\xi)$ of the function $f(t)=\max _{\mathbb{T}_{t}} k^{2}=\varepsilon(t) /(T-t)$, with $\lim _{t \rightarrow T} \varepsilon(t)=0$. Clearly $\widetilde{f}(t) \geq f(t)$, hence also $\widetilde{f}$ goes to $+\infty$ as $t \rightarrow T$.

We notice that since $f$ is locally Lipschitz, the same hold for $\widetilde{f}$, moreover the derivative of this latter, which exists at almost every time $t \in[0, T)$, is zero or coincides with the derivative of $f$, if this happen $\widetilde{f}(t)=f(t)$ also holds.

We saw in inequality (6.2) that when the maximum of $k^{2}$ is taken at some points different from $O$, then $f^{\prime} \leq 2 f^{2}$, so under the same hypothesis, it also holds $\widetilde{f}^{\prime} \leq f^{\prime} \leq 2 f^{2} \leq 2 \widetilde{f}^{2}$.

Let us consider the set $J=\left\{t \in[0, T) \mid \widetilde{f}(t)=\max _{i \in\{1,2,3\}}\left[k^{i}(O, t)\right]^{2}\right\}$, if a value $t$ does not belong to $J$ then either the maximum of $k^{2}$ is taken at some points different from $O$ or $f(t)<\widetilde{f}(t)$ and the derivative of $\widetilde{f}$ at $t$ is zero. In both cases the inequality $\widetilde{f}^{\prime} \leq 2 \widetilde{f}^{2}$ holds at every time such that the derivatives of $f$ and $\tilde{f}$ exist (almost everywhere).

If $T$ is not a limit point of $J$, then definitely $\tilde{f}^{\prime} \leq 2 \widetilde{f}^{2}$ almost everywhere, integrating then the distributional derivative of $1 / \widetilde{f}$, we obtain, as in the previous lemma for $f$, the bound from below $\widetilde{f}(t) \geq \frac{1}{2(T-t)}$.

This gives a contradiction since it implies that definitely $\widetilde{f}>f$, hence $\tilde{f}$ would be constant against the fact that $f(t)$ goes to $+\infty$ as $t \rightarrow T$.

Thus, we can assume that there exists an index $i \in\{1,2,3\}$ and a sequence of times $t_{j} \nearrow T$ belonging to $J$, hence satisfying $\widetilde{f}\left(t_{j}\right)=\left[k^{i}\left(O, t_{j}\right)\right]^{2}$. It follows, by construction, that

$$
k^{2}(p, t) \leq\left[k^{i}\left(O, t_{j}\right)\right]^{2} \text { for every } t \leq t_{j} \text { and } p \in \mathbb{T},
$$

and $\left[k^{i}\left(O, t_{j}\right)\right]^{2} \nearrow+\infty$.

We will deal with the first and the last case of Lemma 6.1 by means of the rescaling procedure of Huisken [25], adapting the line of Stone in [37], [38] to our situation. By means of an argument of Ilmanen in [28, Lemma 8] and [29, Section 3], we will show in Proposition 6.18 that the second case also cannot happen.

We start with the analogue in our situation of Huisken's monotonicity formula for mean curvature flow (see [25]). 
Let $x_{0} \in \mathbb{R}^{2}$ and define the backward heat kernel relative to $\left(x_{0}, T\right)$ as

$$
\rho_{x_{0}}(x, t)=\frac{e^{-\frac{\left|x-x_{0}\right|^{2}}{4(T-t)}}}{\sqrt{4 \pi(T-t)}}
$$

Lemma 6.3. For every $t \in[0, T)$ and $i \in\{1,2,3\}$ the following identity holds

$$
\begin{aligned}
\frac{d}{d t} \int_{\gamma^{i}(\cdot, t)} \rho_{x_{0}}(x, t) d s= & -\int_{\gamma^{i}(\cdot, t)}\left|\underline{k}+\frac{\left(x-x_{0}\right)^{\perp}}{2(T-t)}\right|^{2} \rho_{x_{0}}(x, t) d s \\
& +\left\langle\frac{P^{i}-x_{0}}{2(T-t)} \mid \tau^{i}(1, t)\right\rangle \rho_{x_{0}}\left(P^{i}, t\right) \\
& +\left\langle\frac{\gamma^{i}(0, t)-x_{0}}{2(T-t)} \mid \tau^{i}(0, t)\right\rangle \rho_{x_{0}}\left(\gamma^{i}(0, t), t\right) \\
& +\lambda^{i}(0, t) \rho_{x_{0}}\left(\gamma^{i}(0, t), t\right) .
\end{aligned}
$$

Proof. The proof goes like in [25, Theorem 3.1] with the extra boundary terms coming from the application of the first variation formula (see [34]).

By such formula, for every $C^{1}$ vector field $X$ we have

$\int_{\gamma^{i}(\cdot, t)} \operatorname{div}^{\top} X d s=-\int_{\gamma^{i}(\cdot, t)}\langle X \mid \underline{k}\rangle d s+\left\langle X\left(\gamma^{i}(1, t)\right) \mid \tau^{i}(1, t)\right\rangle-\left\langle X\left(\gamma^{i}(0, t)\right) \mid \tau^{i}(0, t)\right\rangle$

where $\operatorname{div}^{\top}$ means tangential divergence (see [34]), and following Huisken [25],

$$
\begin{aligned}
\frac{d}{d t} \int_{\gamma^{i}(\cdot, t)} \rho_{x_{0}} d s= & \int_{\gamma^{i}(\cdot, t)} \rho_{x_{0}} \operatorname{div}^{\top} \underline{v} d s+\int_{\gamma^{i}(\cdot, t)}\left\langle\nabla \rho_{x_{0}} \mid \underline{v}\right\rangle+\frac{\partial \rho_{x_{0}}}{\partial t} d s \\
= & -\int_{\gamma^{i}(\cdot, t)} \rho_{x_{0}} k^{2}-\operatorname{div}^{\top}\left(\rho_{x_{0}} \lambda \tau\right) d s+\int_{\gamma^{i}(\cdot, t)}\left\langle\nabla^{\perp} \rho_{x_{0}} \mid \underline{v}\right\rangle+\frac{\partial \rho_{x_{0}}}{\partial t} d s \\
= & -\int_{\gamma^{i}(\cdot, t)} \rho_{x_{0}}\left\{k^{2}-\frac{1}{2(T-t)}+\frac{\left\langle x-x_{0} \mid \underline{k}\right\rangle}{2(T-t)}+\frac{\left|x-x_{0}\right|^{2}}{4(T-t)^{2}}\right\} d s \\
& -\lambda^{i}(0, t) \rho_{x_{0}}\left(\gamma^{i}(0, t), t\right) \\
= & -\int_{\gamma^{i}(\cdot, t)}\left|\underline{k}+\frac{x-x_{0}}{2(T-t)}\right|^{2} \rho_{x_{0}} d s-\lambda^{i}(0, t) \rho_{x_{0}}\left(\gamma^{i}(0, t), t\right) \\
& +\int_{\gamma^{i}(\cdot, t)} \frac{\rho_{x_{0}}}{2(T-t)}+\frac{\left\langle x-x_{0} \mid \underline{k}\right\rangle}{2(T-t)} \rho_{x_{0}} d s
\end{aligned}
$$

since $\lambda^{i}(1, t)=0$. 
Now we use again the first variation formula with the field $X(x)=(x-$ $\left.x_{0}\right) \rho_{x_{0}}(x, t) / 2(T-t)$ whose tangential divergence is given explicitly by

$$
\begin{aligned}
\frac{\sum_{l=1}^{2} \nabla_{j}^{\top}\left(\left(x^{l}-x_{0}^{l}\right) \rho_{x_{0}}(x, t)\right)}{2(T-t)} & =\frac{\left\langle x-x_{0} \mid \nabla^{\top} \rho_{x_{0}}(x, t)\right\rangle+\sum_{j=1}^{2}\left\langle e_{j} \mid e_{j}^{\top}\right\rangle \rho_{x_{0}}(x, t)}{2(T-t)} \\
& =\frac{\rho_{x_{0}}(x, t)}{2(T-t)}-\frac{\left|\left(x-x_{0}\right)^{\top}\right|^{2}}{4(T-t)^{2}} \rho_{x_{0}}(x, t)
\end{aligned}
$$

then,

$$
\begin{aligned}
& \int_{\gamma^{i}(\cdot, t)} \operatorname{div}^{\top} X d s=-\int_{\gamma^{i}(\cdot, t)} \frac{\left\langle x-x_{0} \mid \underline{k}\right\rangle}{2(T-t)} \rho_{x_{0}} d s \\
& +\frac{\left\langle P^{i}-x_{0} \mid \tau^{i}(1, t)\right\rangle \rho_{x_{0}}\left(P^{i}, t\right)-\left\langle\gamma^{i}(0, t)-x_{0} \mid \tau^{i}(0, t)\right\rangle \rho_{x_{0}}\left(\gamma^{i}(0, t), t\right)}{2(T-t)} \\
& =\int_{\gamma^{i}(\cdot, t)} \frac{\rho_{x_{0}}}{2(T-t)}-\frac{\left|\left(x-x_{0}\right)^{\top}\right|^{2}}{4(T-t)^{2}} \rho_{x_{0}} d s .
\end{aligned}
$$

Hence,

$$
\begin{aligned}
& \frac{d}{d t} \int_{\gamma^{i}(\cdot, t)} \rho_{x_{0}} d s=-\int_{\gamma^{i}(\cdot, t)}\left|\underline{k}+\frac{x-x_{0}}{2(T-t)}\right|^{2} \rho_{x_{0}} d s-\lambda^{i}(0, t) \rho_{x_{0}}\left(\gamma^{i}(0, t), t\right) \\
& \quad+\frac{\left\langle P^{i}-x_{0} \mid \tau^{i}(1, t)\right\rangle \rho_{x_{0}}\left(P^{i}, t\right)-\left\langle\gamma^{i}(0, t)-x_{0} \mid \tau^{i}(0, t)\right\rangle \rho_{x_{0}}\left(\gamma^{i}(0, t), t\right)}{2(T-t)} \\
& \quad+\int_{\gamma^{i}(\cdot, t)} \frac{\left|\left(x-x_{0}\right)^{\top}\right|^{2}}{4(T-t)^{2}} \rho_{x_{0}} d s \\
& =-\int_{\gamma^{i}(\cdot, t)}\left|\underline{k}+\frac{\left(x-x_{0}\right)^{\perp}}{2(T-t)}\right|^{2} \rho_{x_{0}} d s-\lambda^{i}(0, t) \rho_{x_{0}}\left(\gamma^{i}(0, t), t\right) \\
& \quad+\frac{\left\langle P^{i}-x_{0} \mid \tau^{i}(1, t)\right\rangle \rho_{x_{0}}\left(P^{i}, t\right)-\left\langle\gamma^{i}(0, t)-x_{0} \mid \tau^{i}(0, t)\right\rangle \rho_{x_{0}}\left(\gamma^{i}(0, t), t\right)}{2(T-t)}
\end{aligned}
$$

and reordering the terms we get the claimed identity.

Proposition 6.4 (Monotonicity formula). For every $t \in[0, T)$ the following identity holds

$$
\begin{aligned}
\frac{d}{d t} \int_{\mathbb{T}_{t}} \rho_{x_{0}}(x, t) d s= & -\int_{\mathbb{T}_{t}}\left|\underline{k}+\frac{\left(x-x_{0}\right)^{\perp}}{2(T-t)}\right|^{2} \rho_{x_{0}}(x, t) d s \\
& +\sum_{i=1}^{3}\left\langle\frac{P^{i}-x_{0}}{2(T-t)} \mid \tau^{i}(1, t)\right\rangle \rho_{x_{0}}\left(P^{i}, t\right) .
\end{aligned}
$$


Integrating between $t_{1}$ and $t_{2}$ with $0 \leq t_{1} \leq t_{2}<T$ we get

$$
\begin{aligned}
\int_{t_{1}}^{t_{2}} \int_{\mathbb{T}_{t}} & \left|\underline{k}+\frac{\left(x-x_{0}\right)^{\perp}}{2(T-t)}\right|^{2} \rho_{x_{0}}(x, t) d s d t \\
= & \int_{\mathbb{T}_{t_{1}}} \rho_{x_{0}}\left(x, t_{1}\right) d s-\int_{\mathbb{T}_{t_{2}}} \rho_{x_{0}}\left(x, t_{2}\right) d s \\
& +\sum_{i=1}^{3} \int_{t_{1}}^{t_{2}}\left\langle\frac{P^{i}-x_{0}}{2(T-t)} \mid \tau^{i}(1, t)\right\rangle \rho_{x_{0}}\left(P^{i}, t\right) d t .
\end{aligned}
$$

Proof. We simply add the contributions for the three curves given by Lemma 6.3, recalling that $\gamma^{i}(0, t)=O$ and $\sum_{i=1}^{3} \tau^{i}(0, t)=\sum_{i=1}^{3} \lambda^{i}(0, t)=0$.

Lemma 6.5. Setting $\left|P^{i}-x_{0}\right|=d^{i}$, for every index $i \in\{1,2,3\}$ the following estimate holds

$$
\left|\int_{t}^{T}\left\langle\frac{P^{i}-x_{0}}{2(T-\xi)} \mid \tau^{i}(1, \xi)\right\rangle \rho_{x_{0}}\left(P^{i}, \xi\right) d \xi\right| \leq \frac{1}{\sqrt{2 \pi}} \int_{d^{i} / \sqrt{2(T-t)}}^{+\infty} e^{-y^{2} / 2} d y \leq 1 / 2 .
$$

Then, for every $x_{0} \in \mathbb{R}^{2}$,

$$
\lim _{t \rightarrow T} \sum_{i=1}^{3} \int_{t}^{T}\left\langle\frac{P^{i}-x_{0}}{2(T-\xi)} \mid \tau^{i}(1, \xi)\right\rangle \rho_{x_{0}}\left(P^{i}, \xi\right) d \xi=0 .
$$

Proof. If $d^{i}>0$, we estimate

$$
\begin{aligned}
& \left|\int_{t}^{T}\left\langle\frac{P^{i}-x_{0}}{2(T-\xi)} \mid \tau^{i}(1, \xi)\right\rangle \rho_{x_{0}}\left(P^{i}, \xi\right) d \xi\right| \\
& \quad \leq \int_{t}^{T}\left|\left\langle\frac{P^{i}-x_{0}}{2(T-\xi)} \mid \tau^{i}(1, \xi)\right\rangle\right| \rho_{x_{0}}\left(P^{i}, \xi\right) d \xi \\
& \quad \leq \frac{1}{\sqrt{2 \pi}} \int_{t}^{T} \frac{d^{i}}{[2(T-\xi)]^{3 / 2}} e^{-\frac{\left[d^{i}\right]^{2}}{4(T-\xi)}} d \xi \\
& \quad=\frac{1}{\sqrt{2 \pi}} \int_{d^{i} / \sqrt{2(T-t)}}^{+\infty} e^{-y^{2} / 2} d y \\
& \quad \leq \frac{1}{\sqrt{2 \pi}} \int_{0}^{+\infty} e^{-y^{2} / 2} d y=1 / 2
\end{aligned}
$$

where we could change variable $y=d^{i} / \sqrt{2(T-\xi)}$ since $d^{i}>0$.

Since the integral on $[0,+\infty)$ is finite, looking at the third line we have also that

$$
\lim _{t \rightarrow T}\left|\int_{t}^{T}\left\langle\frac{P^{i}-x_{0}}{2(T-\xi)} \mid \tau^{i}(1, \xi)\right\rangle \rho_{x_{0}}\left(P^{i}, \xi\right) d \xi\right|=0 .
$$

In the special case $d^{i}=0$, that is, $x_{0}$ coincides with the end point $P^{i}$, the corresponding integral is zero for every $t \in[0, T)$, so the thesis follows. 

exists.

Proposition 6.6. For every $x_{0} \in \mathbb{R}^{2}$ the limit $\lim _{t \rightarrow T} \int_{\mathbb{T}_{t}} \rho_{x_{0}}(x, t) d s$ there

Proof. Fixed $x_{0} \in \mathbb{R}^{2}$ we look at the function $b:[0, T) \rightarrow \mathbb{R}$

$$
b(t)=\int_{t}^{T} \sum_{i=1}^{3}\left\langle\frac{P^{i}-x_{0}}{2(T-\xi)} \mid \tau^{i}(1, \xi)\right\rangle \rho_{x_{0}}\left(P^{i}, \xi\right) d \xi
$$

As Lemma 6.5 says that $b$ is bounded and $\lim _{t \rightarrow T} b(t)=0$, the monotonicity formula (6.3) implies that the limit of the statement there exists.

Now, we introduce the the rescaling procedure of Huisken [25].

Fixed $x_{0} \in \mathbb{R}^{2}$, let $\widetilde{F}_{x_{0}}: \mathbb{T} \times[-1 / 2 \log T,+\infty) \rightarrow \mathbb{R}^{2}$ be the map

$$
\widetilde{F}_{x_{0}}(p, \mathfrak{t})=\frac{F(p, t)-x_{0}}{\sqrt{2(T-t)}} \quad \mathfrak{t}(t)=-\frac{1}{2} \log (T-t)
$$

then, the rescaled triods are given by

$$
\widetilde{\mathbb{T}}_{x_{0}, t}=\frac{\mathbb{T}_{t}-x_{0}}{\sqrt{2(T-t)}}
$$

and they evolve according to the equation

$$
\frac{\partial}{\partial \mathfrak{t}} \widetilde{F}_{x_{0}}(p, \mathfrak{t})=\underline{\widetilde{v}}(p, \mathfrak{t})+\widetilde{F}_{x_{0}}(p, \mathfrak{t})
$$

where

$$
\underline{\widetilde{v}}(p, \mathfrak{t})=\frac{\underline{v}(p, t(\mathfrak{t}))}{\sqrt{2(T-t(\mathfrak{t}))}}=\underline{\tilde{k}}+\underline{\tilde{\lambda}}=\tilde{k} v+\tilde{\lambda} \tau \quad \text { and } \quad t(\mathfrak{t})=T-e^{-2 \mathfrak{t}} .
$$

Notice that we did not put the "tilde" over the unit tangent and normal, since they remain the same in the rescaling.

We will often write $\widetilde{O}(\mathfrak{t})=\widetilde{F}_{x_{0}}(O, \mathfrak{t})$ for the 3 -point of the rescaled triod $\widetilde{\mathbb{T}}_{x_{0}, \mathfrak{t}}$, when there is no ambiguity on the point $x_{0}$.

The rescaled curvature evolves according to the following equation,

$$
\partial_{\mathfrak{t}} \widetilde{k}=\widetilde{k}_{\sigma \sigma}+\widetilde{k}_{\sigma} \widetilde{\lambda}+\widetilde{k}^{3}-\widetilde{k}
$$

which can be obtained as in Section 3 by means of the commutation law

$$
\partial_{\mathfrak{t}} \partial_{\sigma}=\partial_{\sigma} \partial_{\mathfrak{t}}+\left(\widetilde{k}^{2}-\tilde{\lambda}_{\sigma}-1\right) \partial_{\sigma}
$$

where we denoted with $\sigma$ the arclength parameter for $\widetilde{\mathbb{T}}_{x_{0}, \mathrm{t}}$.

By a straightforward computation ([25], [38, Lemma 2.3]) we have the following rescaled version of the monotonicity formula. 
Proposition 6.7 (Rescaled Monotonicity Formula). Let $x_{0} \in \mathbb{R}^{2}$ and set

$$
\widetilde{\rho}(x)=e^{-\frac{|x|^{2}}{2}}
$$

For every $\mathrm{t} \in[-1 / 2 \log T,+\infty)$ the following identity holds

$$
\begin{aligned}
\frac{d}{d \mathfrak{t}} \int_{\widetilde{\mathbb{T}}_{x_{0}, \mathfrak{t}}} \widetilde{\rho}(x) d \sigma= & -\int_{\widetilde{\mathbb{T}}_{x_{0}, \mathfrak{t}}}\left|\underline{\widetilde{k}}+x^{\perp}\right|^{2} \widetilde{\rho}(x) d \sigma \\
& +\sum_{i=1}^{3}\left\langle\widetilde{P}_{x_{0}, \mathfrak{t}}^{i} \mid \tau^{i}(1, t(\mathfrak{t}))\right\rangle \widetilde{\rho}\left(\widetilde{P}_{x_{0}, \mathfrak{t}}^{i}\right)
\end{aligned}
$$

where $\widetilde{P}_{x_{0}, \mathfrak{t}}^{i}=\frac{P^{i}-x_{0}}{\sqrt{2(T-t(\mathfrak{t}))}}$. Integrating between $\mathfrak{t}_{1}$ and $\mathfrak{t}_{2}$ with $-1 / 2 \log T \leq \mathfrak{t}_{1} \leq$ $\mathfrak{t}_{2}<+\infty$ we get

$$
\begin{aligned}
\int_{\mathfrak{t}_{1}}^{\mathfrak{t}_{2}} \int_{\widetilde{\mathbb{T}}_{x_{0}, \mathfrak{t}}}\left|\underline{\tilde{k}}+x^{\perp}\right|^{2} \widetilde{\rho}(x) d \sigma d \mathfrak{t}= & \int_{\widetilde{\mathbb{T}}_{x_{0}, \mathfrak{t}_{1}}} \widetilde{\rho}(x) d \sigma-\int_{\widetilde{T}_{x_{0}, \mathfrak{t}_{2}}} \tilde{\rho}(x) d \sigma \\
& +\sum_{i=1}^{3} \int_{\mathfrak{t}_{1}}^{\mathfrak{t}_{2}}\left\langle\widetilde{P}_{x_{0}, \mathfrak{t}}^{i} \mid \tau^{i}(1, t(\mathfrak{t}))\right\rangle \widetilde{\rho}\left(\widetilde{P}_{x_{0}, \mathfrak{t}}^{i}\right) d \mathfrak{t} .
\end{aligned}
$$

Then, we have the analog of Lemma 6.5 whose proof follows in the same way, substituting the rescaled quantities.

LEMMA 6.8. For every index $i \in\{1,2,3\}$ the following estimate holds

$$
\left|\int_{\mathfrak{t}}^{+\infty}\left\langle\widetilde{P}_{x_{0}, \xi}^{i} \mid \tau^{i}(1, t(\xi))\right\rangle \widetilde{\rho}\left(\widetilde{P}_{x_{0}, \xi}^{i}\right) d \xi\right| \leq \sqrt{\pi / 2} .
$$

Then, for every $x_{0} \in \mathbb{R}^{2}$,

$$
\lim _{\mathfrak{t} \rightarrow+\infty} \sum_{i=1}^{3} \int_{\mathfrak{t}}^{+\infty}\left\langle\widetilde{P}_{x_{0}, \xi}^{i} \mid \tau^{i}(1, t(\xi))\right\rangle \widetilde{\rho}\left(\widetilde{P}_{x_{0}, \xi}^{i}\right) d \xi=0
$$

We need the following lemmas in order to study the possible limits of the rescaled triods.

LEMMA 6.9. Under the Type I hypothesis (6.1) there exists $\lim _{t \rightarrow T} F(O, t)=$ $\widehat{O} \in \mathbb{R}^{2}$.

The 3-point $\widetilde{F}_{x_{0}}(O, \mathfrak{t})$ of the rescaled triods either it is uniformly bounded or it goes to infinity as $\mathfrak{t} \rightarrow+\infty$, according to the fact that $x_{0}=\widehat{O}$ or not. 
Proof. Since at the 3 -point we have $\sum_{i=1}^{3}\left(\lambda^{i}\right)^{2}=\sum_{i=1}^{3}\left(k^{i}\right)^{2} \leq C /(T-t)$ we have also $|\underline{v}(O, t)|^{2} \leq C /(T-t)$ for some constant $C>0$ independent of $t \in[0, T)$. Then we get

$$
\begin{aligned}
\left|F\left(O, t_{1}\right)-F\left(O, t_{2}\right)\right| & =\left|\int_{t_{1}}^{t_{2}} \frac{\partial F}{\partial t} d t\right| \\
& \leq \int_{t_{1}}^{t_{2}}|\underline{v}(O, t)| d t \leq \int_{t_{1}}^{t_{2}} \sqrt{\frac{C}{T-t}} d t \leq 2 \sqrt{C\left(T-t_{1}\right)}
\end{aligned}
$$

for every $0 \leq t_{1} \leq t_{2}<T$. Hence, the $\operatorname{limit}_{t \rightarrow T} F(O, t)=\widehat{O}$ there exists and

$$
|F(O, t)-\widehat{O}| \leq 2 \sqrt{C(T-t)}
$$

for every $t$.

Considering then the 3-points $\widetilde{F}_{\widehat{O}}(O, \mathfrak{t})$ of the triods $\widetilde{\mathbb{T}}_{\widehat{O}}$, , we have

$$
\left|\widetilde{F}_{\widehat{O}}(O, \mathfrak{t})\right|=\frac{|F(O, t)-\widehat{O}|}{\sqrt{2(T-t)}} \leq \frac{2 \sqrt{C(T-t)}}{\sqrt{2(T-t)}}=\sqrt{2 C}
$$

hence, if $x_{0}=\widehat{O}$ then the 3-point always belongs to the ball $B \sqrt{2 C}$.

By the same inequality (6.9) it follows that if $x_{0} \neq \widehat{O}$ then the 3-points of the rescaled triods go to infinity as $t \rightarrow+\infty$.

Lemma 6.10. The curvature $\widetilde{k}$ of the rescaled triods $\widetilde{\mathbb{T}}_{x_{0}, t}$ is uniformly bounded in space and time. Moreover, for every ball $B_{R}$ centered at the origin of $\mathbb{R}^{2}$, we have the following estimates with a constant $C_{R}$ independent of $x_{0} \in \mathbb{R}^{2}$ and $\mathfrak{t} \in$ $[-1 / 2 \log T,+\infty)$

$$
\mathcal{H}^{1}\left(\widetilde{\mathbb{T}}_{x_{0}, t} \cap B_{R}\right) \leq C_{R} .
$$

PROOF. The maximum of the curvature of every rescaled triod is bounded by a uniform constant, by the assumption (6.1) on the blow up rate of the curvature.

Moreover, using the rescaled monotonicity formula (6.7) we get

$$
\begin{aligned}
\int_{\widetilde{\mathbb{T}}_{x_{0}, \mathrm{t}}} \tilde{\rho} d \sigma= & \int_{\widetilde{\mathbb{T}}_{x_{0},-1 / 2 \log T}} \tilde{\rho} d \sigma-\int_{-1 / 2 \log T \widetilde{\mathbb{T}}_{x_{0}, \xi}}^{\mathrm{t}}\left|\underline{\tilde{k}}+x^{\perp}\right|^{2} \widetilde{\rho} d \sigma d \xi \\
& +\sum_{i=1}^{3} \int_{-1 / 2 \log T}^{\mathfrak{t}}\left\langle\widetilde{P}_{x_{0}, \xi}^{i} \mid \tau^{i}(1, t(\xi))\right\rangle \widetilde{\rho}\left(\widetilde{P}_{x_{0}, \xi}^{i}\right) d \xi \leq C
\end{aligned}
$$

where the last estimate follows from Lemma 6.8 .

Hence, since $\widetilde{\rho} \geq e^{-R^{2} / 2}$ in every ball $B_{R}$ centered at the origin of $\mathbb{R}^{2}$, we have a uniform bound $\mathcal{H}^{1}\left(\widetilde{\mathbb{T}}_{x_{0}, \mathrm{t}} \cap B_{R}\right) \leq C_{R}$ for some constants $C_{R}$ independent of $\mathfrak{t}$ and $x_{0}$. 
Definition 6.11. We say that a sequence of triods converges in the $C_{\text {loc }}^{r}$ topology if, after reparametrizing the curves composing the triods in arclength, they converge in $C^{r}$ in every compact of $\mathbb{R}^{2}$.

The definition of convergence in $W_{\mathrm{loc}}^{n, p}$ is analogous.

LEMMA 6.12. The function (see Section 4)

$$
E(\mathbb{T})=\inf _{\substack{p, q \in \mathbb{T} \\ p \neq q}} \frac{|p-q|^{2}}{A_{p, q}},
$$

defined on the class of $C^{2}$ triods without self-intersections (bounded or unbounded and with or without end points), is upper semicontinuous with respect to the $C_{\mathrm{loc}}^{2}$ convergence.

Moreover, $E$ is dilation and translation invariant.

Hence, every $C_{\mathrm{loc}}^{2}$ limit of rescaled triods satisfies $E>C>0$ where the uniform positive constant $C$ is given by Theorem 4.6. Thus, such limit has no self-intersections.

PROOF. The proof is straightforward.

Lemma 6.13. For every $x_{0} \in \mathbb{R}^{2}$ and every sequence of rescaled times $\mathfrak{t}_{j} \rightarrow \infty$ there exists a subsequence $\mathfrak{t}_{j_{l}}$ such that the triods $\widetilde{\mathbb{T}}_{x_{0}, t_{j_{l}}}$ converge in the $C_{\text {loc }}^{1}$ topology to a limit set $\mathbb{T}_{\infty}$ which, if non empty, is a curve or a triod with at most one end point and without self-intersections.

Moreover, the Radon measures $\mathcal{H}^{1}\left\llcorner\widetilde{\mathbb{T}}_{x_{0}, \mathrm{t}_{j l}}\right.$ weakly converge in $\mathbb{R}^{2}$ to the Radon measure $\mathcal{H}^{1}\left\llcorner\mathbb{T}_{\infty}\right.$.

PROOF. Reparametrizing the triods in arclength, we have curves with uniformly bounded first and second derivatives, moreover, by Lemma 6.10, for every ball $B_{R}$ centered at the origin of $\mathbb{R}^{2}$ we have a uniform bound $\mathcal{H}^{1}\left(\widetilde{\mathbb{T}}_{x_{0}, \mathrm{t}} \cap B_{R}\right) \leq$ $C_{R}$ for some constants $C_{R}$ independent of $\mathrm{t}$.

Then, by standard compactness arguments (see [25], [31], 34]), the sequence $\widetilde{\mathbb{T}}_{x_{0}, \mathrm{t}_{j}}$ of reparametrized triods has a subsequence $\widetilde{\mathbb{T}}_{x_{0}, \mathrm{t}_{j_{l}}}$ weakly ${ }^{\star}$ converging in $W_{\text {loc }}^{2, \infty}$, then in the $C_{\text {loc }}^{1}$ topology to a (possibly empty) set $\mathbb{T}_{\infty}$ which, if $x_{0}$ is distinct from all the $P^{i}$, has no end points since they go to infinity during the rescaled flow. If $x_{0}=P^{i}$, the set $\mathbb{T}_{\infty}$ has a single end point at the origin of $\mathbb{R}^{2}$.

In both cases the 3-point could be present or not, if it is present then the angles are of 120 degrees by the convergence of the curves in $C_{\text {loc }}^{1}$. The only "strange" situation is if $x_{0}=P^{1}$, for instance, and $\lim _{l \rightarrow \infty} \widetilde{O}\left(\mathfrak{t}_{j_{l}}\right)=0$, which is in contradiction with the fact that the three lengths are uniformly bounded away from zero, indeed in this situation the curve between $P^{1}$ and the 3-point has to collapse otherwise embeddedness, which we are going to show now, is lost.

The limit set, which we suppose non empty, has no self-intersections by Lemma 6.12.

Finally, the embeddedness of the limit and the $C^{1}$ convergence in every compact imply that the Radon measures $\mathcal{H}^{1}\left\llcorner\widetilde{\mathbb{T}}_{x_{0}, t_{j l}}\right.$ weakly ${ }^{\star}$ converge in $\mathbb{R}^{2}$ to the Radon measure $\mathcal{H}^{1}\left\llcorner\mathbb{T}_{\infty}\right.$. 
LEMMA 6.14. If the 3-point of the rescaled triod $\widetilde{\mathbb{T}}_{x_{0}, t}$ does not belong to the ball $B_{2 R}(z) \subset \mathbb{R}^{2}$ with radius $2 R$ and center $z \in \mathbb{R}^{2}$, then there exist constants $\delta_{R}>0$ and $D_{R}$, independent of the points $z, x_{0} \in \mathbb{R}^{2}$ and the time $\mathfrak{t} \in[-1 / 2 \log T,+\infty)$, such that $\widetilde{k}_{\sigma}, \widetilde{k}_{\sigma \sigma}$ and $\widetilde{k}_{\mathfrak{t}}$ for the family $\left\{\widetilde{\mathbb{T}}_{x_{0}, r} \mid r \in\left[\mathfrak{t}, \mathfrak{t}+\delta_{R}\right]\right\}$ are uniformly bounded by $D_{R}$ in the smaller ball $B_{R}(z)$.

Proof. By the control on the curvature, the velocity of the 3-point is bounded by a uniform constant, hence, for some $\delta_{R}>0$ in the time interval $\left[\mathfrak{t}-\delta_{R}, \mathfrak{t}+\delta_{R}\right]$ the 3 -point cannot enter in the ball $B_{3 R / 2}(z)$.

Then, as the 3-points are far from the ball, by the uniform bound on the curvature of all the rescaled triods, repeating the interior estimates of Ecker and Huisken in [17] (see also [16, Section 2]) for the rescaled flow and recalling the evolution equation for the rescaled curvature $\partial_{\mathrm{t}} \widetilde{k}=\widetilde{k}_{\sigma \sigma}+\widetilde{\eta} \widetilde{k}_{\sigma}+\widetilde{k}^{3}-\widetilde{k}$, we get the thesis, possibly choosing a smaller $\delta_{R}>0$.

REMARK 6.15. The same conclusion clearly holds for a family of triods moving by curvature (not rescaled) if we have a uniform bound on the curvature.

PROPOSITION 6.16. For every $x_{0} \in \mathbb{R}^{2}$ and every sequence of rescaled times $\mathfrak{t}_{j} \rightarrow \infty$ there exists a subsequence $\mathfrak{t}_{j_{l}}$ such that the triods $\widetilde{\mathbb{T}}_{x_{0}, \mathfrak{t}_{j_{l}}}$ converge in the $C_{\text {loc }}^{2}$ topology to a limit set $\mathbb{T}_{\infty}$ which, if non empty, is one of the following:

- a triod composed of three halfines originating from $0 \in \mathbb{R}^{2}$,

- a halfline from $0 \in \mathbb{R}^{2}$,

- a straight line from $0 \in \mathbb{R}^{2}$.

Moreover, the Radon measures $\mathcal{H}^{1}\left\llcorner\widetilde{\mathbb{T}}_{x_{0}, t_{j_{l}}}\right.$ weakly ${ }^{\star}$ converge in $\mathbb{R}^{2}$ to the Radon measure $\mathcal{H}^{1}\left\llcorner\mathbb{T}_{\infty}\right.$.

Proof. By Lemma 6.13, we have to show that, supposing the $C_{\text {loc }}^{1}$-limit $\mathbb{T}_{\infty}=\lim _{l \rightarrow \infty} \widetilde{\mathbb{T}}_{x_{0}, t_{j l}}$ non empty, it is among the ones of the statement.

Putting $\mathfrak{t}_{1}=-1 / 2 \log T$ and sending $\mathfrak{t}_{2}$ to $+\infty$ in the rescaled monotonicity formula (6.8), by Lemma 6.5 we get

$$
\int_{-1 / 2 \log T \widetilde{\mathbb{T}}_{x_{0}, \mathfrak{t}}}^{+\infty}\left|\underline{\tilde{k}}+x^{\perp}\right|^{2} \widetilde{\rho} d \sigma d \mathfrak{t}<+\infty,
$$

hence, extracting from the sequence of times $\mathfrak{t}_{j_{l}}$ a subsequence (not relabelled) with $\mathfrak{t}_{j_{l+1}}>\mathfrak{t}_{j_{l}}+1 / l$, we see that there exists an increasing sequence $r_{j_{l}}$ such that $\mathfrak{t}_{j_{l}} \leq r_{j_{l}} \leq \mathfrak{t}_{j_{l}}+1 / l$ and on a subsequence of the $r_{j_{l}}$ (again not relabelled) we have

$$
\lim _{l \rightarrow \infty} \int_{\widetilde{\mathbb{T}}_{x_{0}, r_{j_{l}}}}\left|\underline{\tilde{k}}+x^{\perp}\right|^{2} \widetilde{\rho} d \sigma^{1}=0 .
$$


Reapplying Lemma 6.13, we can assume that also the triods $\widetilde{\mathbb{T}}_{x_{0}, r_{j_{l}}}$ converges to some limit $\overline{\mathbb{T}}_{\infty}$ in $W_{\text {loc }}^{2, \infty}$ (possibly empty), and since the integral above is lower semicontinuous in this convergence (see [34]), the limit $\mathbb{T}_{\infty}$ satisfies $\underline{\underline{k}}+x^{\perp}=0$, distributionally. The limit set is composed of curves in $W_{\text {loc }}^{2, \infty}$ but from the relation $\underline{\underline{k}}+x^{\perp}=0$ it follows that $\underline{\underline{k}}$ is continuous, since the curves are $C_{\mathrm{loc}}^{1}$

Such limit set is a triod or a curve and the end point is present or not according to the choice of the point $x_{0}$.

As the relation above implies $\widetilde{k}=-\langle x \mid v\rangle$ at every point $x \in \overline{\mathbb{T}}_{\infty}$, the classification Lemmas 5.2, 5.3, 5.4 show that in any case the curvature of the limit set is zero everywhere and that $\overline{\mathbb{T}}_{\infty}$ is among the sets of the statement. Indeed, when such limit is an halfline, we have necessarily that the point $x_{0}$ coincides with one of the fixed end points of the triods, hence the limit halfline starts from the origin of $\mathbb{R}^{2}$.

We show now that $\mathbb{T}_{\infty}=\overline{\mathbb{T}}_{\infty}$ and that the convergence is in the $C_{\text {loc }}^{2}$ topology, proving the proposition.

We consider a point $y \in \mathbb{T}_{\infty}$ such that the distance $\operatorname{dist}\left(y, \overline{\mathbb{T}}_{\infty}\right)>0$. If we denote with $y_{\mathfrak{t}}=\widetilde{F}_{x_{0}}\left(p_{\mathfrak{t}}, \mathfrak{t}\right)$ the point of minimum distance from $y$ in the rescaled triod $\widetilde{\mathbb{T}}_{x_{0}, \mathfrak{t}}$ and we look at the function $f(\mathfrak{t})=\operatorname{dist}\left(y, \widetilde{\mathbb{T}}_{x_{0}, \mathfrak{t}}\right)=\left|y-y_{\mathfrak{t}}\right|$ we have (with the usual remarks about differentiability),

$$
\begin{aligned}
\frac{d f(\mathfrak{t})}{d \mathfrak{t}} & =\frac{\left\langle y-y_{\mathfrak{t}} \mid \frac{\partial \widetilde{F}_{x_{0}}\left(p_{\mathfrak{t}}, \mathfrak{t}\right)}{\partial \mathfrak{t}}\right\rangle}{\left|y-y_{\mathfrak{t}}\right|} \\
& =\frac{\left\langle y-y_{\mathfrak{t}} \mid \underline{\widetilde{v}}\left(p_{\mathfrak{t}}, \mathfrak{t}\right)+\widetilde{F}_{x_{0}}\left(p_{\mathfrak{t}}, \mathfrak{t}\right)\right\rangle}{\left|y-y_{\mathfrak{t}}\right|} \leq C|\widetilde{k}|+\left|\widetilde{F}_{x_{0}}\left(p_{\mathfrak{t}}, \mathfrak{t}\right)\right|
\end{aligned}
$$

where we substituted the velocity with the curvature since, if $y_{t}$ is in the interior, the vector $\left(y-y_{\mathfrak{t}}\right)$ is orthogonal to $\widetilde{\mathbb{T}}_{x_{0}, \mathfrak{t}}$ by minimality, if $y_{\mathrm{t}}$ is an end point then $\widetilde{\underline{v}}\left(p_{\mathfrak{t}}, \mathfrak{t}\right)=0$, finally if $y_{\mathfrak{t}}$ is the 3-point, by the usual relation $\sum_{i=1}^{3}\left(\widetilde{\lambda}^{i}\right)^{2}=\sum_{i=1}^{3}\left(\widetilde{k}^{i}\right)^{2}$, the velocity is controlled by a constant multiple of the curvature.

Since $\widetilde{k}$ is uniformly bounded and the triangular inequality gives $\left|\widetilde{F}_{x_{0}}\left(p_{\mathfrak{t}}, \mathfrak{t}\right)\right|=$ $\left|y_{\mathfrak{t}}\right| \leq|y|+\left|y-y_{\mathfrak{t}}\right| \leq C+f(\mathfrak{t})$, we conclude

$$
\frac{d f(\mathfrak{t})}{d \mathfrak{t}} \leq f(\mathfrak{t})+C .
$$

Integrating this differential inequality on the interval $\left[\mathfrak{t}_{j_{l}}, r_{j_{l}}\right]$ we get

$$
f\left(r_{j_{l}}\right) \leq e^{r_{j_{l}}-\mathfrak{t}_{l}}\left(f\left(\mathfrak{t}_{j_{l}}\right)+C\right)-C \leq e^{1 / l} f\left(\mathfrak{t}_{j_{l}}\right)+C\left(e^{1 / l}-1\right)
$$

so, if $l \rightarrow \infty$, since we know that $\lim _{l \rightarrow \infty} f\left(\mathfrak{t}_{j_{l}}\right)=0$ we have also $\lim _{l \rightarrow \infty} f\left(r_{j_{l}}\right)=$ 0 , thus $y \in \overline{\mathbb{T}}_{\infty}$. 
This clearly implies that $\overline{\mathbb{T}}_{\infty}$ cannot be empty, then, inverting the roles of the two limit sets and repeating this argument we conclude that they must coincide.

Now we show the $C_{\text {loc }}^{2}$ convergence.

If the limit set $\mathbb{T}_{\infty}$ is a straight line, by the $C^{1}$ convergence in every ball $B_{R}$ and the uniform bound on the curvature $\widetilde{k}$, we can apply Lemma 6.14 to get a uniform bound on the norm $\left\|\widetilde{k}_{\sigma}\right\|_{L^{\infty}\left(B_{R}\right)}$ for all the triods $\widetilde{\mathbb{T}}_{x_{0}, \mathrm{t}_{j}}$, hence the $C_{\text {loc }}^{2}$ convergence follows.

With the same argument we have a uniform bound on $\widetilde{k}_{\sigma}$ in the subset $B_{n R} \backslash B_{2 R}$, for every $n \in \mathbb{N}$ greater than 2, in the case that the 3-point of $\widetilde{\mathbb{T}}_{x_{0}, \mathrm{t}}$ definitely belongs to the ball $B_{R}$ (see Lemma 6.9), or the limit $\mathbb{T}_{\infty}$ is a halfline from the origin of $\mathbb{R}^{2}$.

Then, we work out a local version of the estimates leading to Proposition 3.17 in order to deal with these two situations. By means of equations (6.5) and (6.6) we have

$$
\begin{aligned}
\partial_{\mathrm{t}} \widetilde{k} & =\widetilde{k}_{\sigma \sigma}+\widetilde{k}_{\sigma} \widetilde{\lambda}+\widetilde{k}^{3}-\widetilde{k} \\
\partial_{\mathrm{t}} \widetilde{k}_{\sigma} & =\widetilde{k}_{\sigma \sigma \sigma}+\widetilde{k}_{\sigma \sigma} \widetilde{\lambda}+4 \widetilde{k}^{2} \widetilde{k}_{\sigma}-2 \widetilde{k}_{\sigma} \\
\partial_{\mathrm{t}} \widetilde{k}_{\sigma \sigma} & =\widetilde{k}_{\sigma \sigma \sigma \sigma}+\widetilde{\lambda} \widetilde{k}_{\sigma \sigma \sigma}+5 \widetilde{k}^{2} \widetilde{k}_{\sigma \sigma}+8 \widetilde{k}^{2}-3 \widetilde{k}_{\sigma \sigma}
\end{aligned}
$$

hence, for every smooth function $\varphi: \mathbb{R}^{2} \times[0, \delta] \rightarrow[0,1]$ with compact support we compute,

$$
\begin{aligned}
& \frac{d}{d \mathfrak{t}} \int_{\widetilde{\mathbb{T}}_{x_{0}, \mathfrak{t}}}\left(\widetilde{k}^{2}+\widetilde{k}_{\sigma}^{2}+\mathfrak{t}^{2} \widetilde{k}_{\sigma \sigma}^{2} / 2\right) \varphi^{2} d \sigma=-\int_{\widetilde{\mathbb{T}}_{x_{0}, \mathfrak{t}}}\left(\widetilde{k}_{\sigma}^{2}+\mathfrak{t} \widetilde{k}_{\sigma \sigma}^{2}+\mathfrak{t}^{2} \widetilde{k}_{\sigma \sigma \sigma}^{2}\right) \varphi^{2} d \sigma \\
& -\varphi^{2}(\widetilde{O}, \mathfrak{t}) \sum_{i=1}^{3} 2 \widetilde{k}_{k_{\sigma}}+2 \mathfrak{t} \widetilde{k}_{\sigma} \widetilde{k}_{\sigma \sigma}+\left.\mathfrak{t}^{2} \widetilde{k}_{\sigma \sigma} \widetilde{k}_{\sigma \sigma \sigma}\right|_{\text {at the 3-point }} \\
& +\int_{\widetilde{\mathbb{T}}_{x_{0}, \mathrm{t}}}\left(2 \widetilde{k} \widetilde{k}_{\sigma}+2 \mathfrak{t} \widetilde{k}_{\sigma} \widetilde{k}_{\sigma \sigma}+\mathfrak{t}^{2} \widetilde{k}_{\sigma \sigma} \widetilde{k}_{\sigma \sigma \sigma}\right) \tilde{\lambda} \varphi^{2} d \sigma \\
& +\int_{\widetilde{\mathbb{T}}_{x_{0}, \mathfrak{t}}}\left(\widetilde{k}^{2}+\widetilde{\mathfrak{k}}_{\sigma}^{2}+\mathfrak{t}^{2} \widetilde{k}_{\sigma \sigma}^{2} / 2\right)\left(\varphi^{2} \widetilde{\lambda}_{\sigma}+2 \varphi\langle\nabla \varphi \mid \tau\rangle \widetilde{\lambda}\right) d \sigma \\
& +\int_{\widetilde{\mathbb{T}}_{x_{0}, \mathrm{t}}}\left(\widetilde{k}^{2}+\widetilde{\mathrm{k}}_{\sigma}^{2}+\mathfrak{t}^{2} \widetilde{k}_{\sigma \sigma}^{2} / 2\right)\left(1-\widetilde{k}^{2}\right) \varphi^{2} d \sigma \\
& +2 \underset{\widetilde{T}_{x_{0}, \mathfrak{t}}}{\int}\left[\widetilde{k}^{4}-\widetilde{k}^{2}+\mathfrak{t}\left(4 \widetilde{k}^{2} \widetilde{k}_{\sigma}^{2}-2 \widetilde{k}_{\sigma}^{2}\right)+\frac{\mathfrak{t}^{2}}{2}\left(8 \widetilde{k} \widetilde{k}_{\sigma}^{2} \widetilde{k}_{\sigma \sigma}-3 \widetilde{k}_{\sigma \sigma}^{2}\right)\right] \varphi^{2} d \sigma \\
& +\int_{\widetilde{\mathbb{T}}_{x_{0}, \mathrm{t}}}\left(\widetilde{k}^{2}+\widetilde{\mathrm{t}}_{\sigma}^{2}+\mathfrak{t}^{2} \widetilde{k}_{\sigma \sigma}^{2} / 2\right)\left(\left\langle\nabla \varphi^{2} \mid \underline{\widetilde{k}}\right\rangle+2 \varphi \varphi_{\mathrm{t}}\right) d \sigma
\end{aligned}
$$

where we already forgot the end points contributions, by Lemma 3.10. 
After integrating by parts the terms containing $\tilde{\lambda}$, if $\left\langle\nabla \varphi^{2} \mid \underline{\widetilde{k}}\right\rangle+2 \varphi \varphi_{\mathrm{t}}$ is non positive for $\mathfrak{t} \in[0, \delta]$ and taking into account that $\widetilde{k}$ is bounded, we get

$$
\begin{aligned}
& \frac{d}{d \mathfrak{t}} \int_{\widetilde{\mathbb{T}}_{x_{0}, \mathfrak{t}}}\left(\widetilde{k}^{2}+\widetilde{t}_{\sigma}^{2}+\mathfrak{t}^{2} \widetilde{k}_{\sigma \sigma}^{2} / 2\right) \varphi^{2} d \sigma \\
& \leq-\int_{\widetilde{\mathbb{T}}_{x_{0}, \mathfrak{t}}}\left(\widetilde{k}_{\sigma}^{2}+\widetilde{t}_{\sigma \sigma}^{2}+\mathfrak{t}^{2} \widetilde{k}_{\sigma \sigma \sigma}^{2}\right) \varphi^{2} d \sigma \\
& -\varphi^{2}(\widetilde{O}, \mathfrak{t}) \sum_{i=1}^{3} 2 \widetilde{k}_{k}+2 \mathfrak{t} \widetilde{k}_{\sigma} \widetilde{k}_{\sigma \sigma}+\left.\mathfrak{t}^{2} \widetilde{k}_{\sigma \sigma} \widetilde{k}_{\sigma \sigma \sigma}\right|_{\text {at the 3-point }} \\
& -\varphi^{2}(\widetilde{O}, \mathfrak{t}) \sum_{i=1}^{3} \widetilde{k}^{2} \widetilde{\lambda}+\mathfrak{t} \widetilde{k}_{\sigma}^{2} \widetilde{\lambda}+\mathfrak{t}^{2} \widetilde{k}_{\sigma \sigma}^{2} \widetilde{\lambda} /\left.2\right|_{\text {at the 3-point }} \\
& +C \underset{\widetilde{\mathbb{T}}_{x_{0}, \mathrm{t}}}{ }\left(\widetilde{k}^{2}+\widetilde{t}_{\sigma}^{2}+\mathfrak{t}^{2} \widetilde{k}_{\sigma \sigma}^{2} / 2\right) \varphi^{2} d \sigma \\
& +2 \underset{\widetilde{\mathbb{T}}_{x_{0}, \mathrm{t}}}{2}\left[\widetilde{k}^{4}-\widetilde{k}^{2}+\mathfrak{t}\left(4 \widetilde{k}^{2} \widetilde{k}_{\sigma}^{2}-2 \widetilde{k}_{\sigma}^{2}\right)+\frac{\mathfrak{t}^{2}}{2}\left(8 \widetilde{k}_{\tilde{k}_{\sigma}^{2}}^{2} \widetilde{k}_{\sigma \sigma}-3 \widetilde{k}_{\sigma \sigma}^{2}\right)\right] \varphi^{2} d \sigma .
\end{aligned}
$$

In doing this we choose a function $\varphi$ as follows. If the 3-point of the rescaled triods is bounded, according to Lemma 6.9, and it is definitely contained in the ball of radius $R$, supposing that the curvature is uniformly bounded by some constant $C>0$, we set $\varphi(x, \mathfrak{t})=\sqrt{h\left(\psi(x)-C \mathfrak{t}\|\nabla \psi\|_{\left.L^{\infty}\right)}\right.}$ choosing a radially monotone and symmetric smooth function $\psi: \mathbb{R}^{2} \rightarrow[0,1]$, with compact support, such that $\left.\psi\right|_{B_{2 R}}=1$ and $\left.\psi\right|_{\mathbb{R}^{2} \backslash B_{3 R}}=0$ and a smooth increasing function $h: \mathbb{R} \rightarrow[0,1]$ such that $h(z)=0$ if $z \leq 0$ and $h(z)=1$ for $z \geq 1$. Then, for $\delta>0$ small enough, depending only on $\|\nabla \psi\|_{L^{\infty}}$ and $C$, the function $\varphi$ satisfies the requirements above and possibly choosing a smaller value $\delta>0$, there holds $\varphi \geq 1 / 2$ on $B_{2 R}$ for every $\mathfrak{t} \in[0, \delta]$.

Since by Lemma 6.9 the 3-point of the rescaled triods is definitely inside or outside the ball $B_{R}$ we can consider $\varphi^{2}(\widetilde{O}, \mathfrak{t})$ constantly equal to one or zero.

Hence, if the 3-point is present, we are dealing with the case where $\mathbb{T}_{\infty}$ is an unbounded triod, so, by the $C_{\text {loc }}^{1}$ convergence, the lengths of the curves in the ball $B_{2 R}$ are bounded from below by $R$ and we can treat the 3-point term as before in proving Proposition 3.17. Then, denoting with $\mathcal{I}$ the boundary 
term, we get

$$
\begin{aligned}
& \frac{d}{d \mathfrak{t}} \int_{\widetilde{\mathbb{T}}_{x_{0}, \mathfrak{t}}}\left(\widetilde{k}^{2}+\widetilde{t}_{\sigma}^{2}+\mathfrak{t}^{2} \widetilde{k}_{\sigma \sigma}^{2} / 2\right) \varphi^{2} d \sigma \leq-\int_{\widetilde{\mathbb{T}}_{x_{0}, \mathfrak{t}}}\left(\widetilde{k}_{\sigma}^{2}+\widetilde{t}_{\sigma \sigma}^{2}+\mathfrak{t}^{2} \widetilde{k}_{\sigma \sigma \sigma}^{2}\right) \varphi^{2} d \sigma+\mathcal{I} \\
& +C \underset{\widetilde{\mathbb{T}}_{x_{0}, \mathfrak{t}}}{\int}\left(\widetilde{k}^{2}+\widetilde{k}_{\sigma}^{2}+\mathfrak{t}^{2} \widetilde{k}_{\sigma \sigma}^{2} / 2\right) \varphi^{2} d \sigma \\
& +\underset{\widetilde{\mathbb{T}}_{x_{0}, \mathrm{t}}}{2 \int}\left[\widetilde{k}^{4}-\widetilde{k}^{2}+\mathfrak{t}\left(4 \widetilde{k}^{2} \widetilde{k}_{\sigma}^{2}-2 \widetilde{k}_{\sigma}^{2}\right)+\frac{\mathfrak{t}^{2}}{2}\left(8 \widetilde{k}_{k_{\sigma}^{2}}^{2} \widetilde{k}_{\sigma \sigma}-3 \widetilde{k}_{\sigma \sigma}^{2}\right)\right] \varphi^{2} d \sigma \\
& \leq-\underset{\widetilde{\mathbb{T}}_{x_{0}, \mathfrak{t}}}{-}\left(\widetilde{k}_{\sigma}^{2}+\widetilde{t}_{\sigma \sigma}^{2}+\mathfrak{t}^{2} \widetilde{k}_{\sigma \sigma \sigma}^{2}\right) \varphi^{2} d \sigma+\mathcal{I}+C+\underset{\widetilde{\mathbb{T}}_{x_{0}, \mathfrak{t}}}{C}\left(\widetilde{k}^{2}+\widetilde{t}_{\sigma}^{2}+\mathfrak{t}^{2} \widetilde{k}_{\sigma \sigma}^{2} / 2\right) \varphi^{2} d \sigma
\end{aligned}
$$

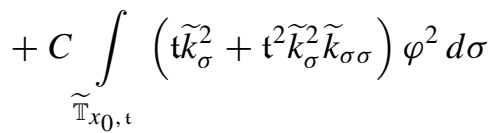

$$
\begin{aligned}
& \leq-\underset{\widetilde{\mathbb{T}}_{x_{0}, \mathfrak{t}}}{-}\left(\widetilde{k}_{\sigma}^{2}+\widetilde{t}_{\sigma \sigma}^{2}+\mathfrak{t}^{2} \widetilde{k}_{\sigma \sigma \sigma}^{2}\right) \varphi^{2} d \sigma+\mathcal{I}+C+\underset{\widetilde{\mathbb{T}}_{x_{0}, \mathfrak{t}}}{C}\left(\widetilde{k}^{2}+\widetilde{t}_{\sigma}^{2}+\mathfrak{t}^{2} \widetilde{k}_{\sigma \sigma}^{2} / 2\right) \varphi^{2} d \sigma \\
& +C \underset{\widetilde{\mathbb{T}}_{x_{0}, \mathfrak{t}}}{\int}\left(\widetilde{\mathrm{k}}_{\sigma}^{2}+\mathfrak{t}^{2} \widetilde{k}_{\sigma \sigma}^{2}+\mathfrak{t}^{2} \widetilde{k}_{\sigma}^{4}\right) \varphi^{2} d \sigma
\end{aligned}
$$

and choosing $\delta<1$ such that $\delta C<1 / 2$, it follows

$$
\begin{aligned}
\frac{d}{d \mathfrak{t}} \int_{\widetilde{\mathbb{T}}_{x_{0}, \mathfrak{t}}}\left(\widetilde{k}^{2}+\widetilde{t}^{2}+\mathfrak{t}^{2} \widetilde{k}_{\sigma \sigma}^{2} / 2\right) \varphi^{2} d \sigma \leq & -1 / 2 \int_{\widetilde{\mathbb{T}}_{x_{0}, \mathfrak{t}}}\left(\widetilde{k}_{\sigma}^{2}+\mathfrak{t} \widetilde{k}_{\sigma \sigma}^{2}+\mathfrak{t}^{2} \widetilde{k}_{\sigma \sigma \sigma}^{2}\right) \varphi^{2} d \sigma+\mathcal{I} \\
& +C+C \int_{\widetilde{\mathbb{T}}_{x_{0}, \mathfrak{t}}}\left(\widetilde{k}^{2}+\widetilde{k}_{\sigma}^{2}+\mathfrak{t}^{2} \widetilde{k}_{\sigma \sigma}^{2} / 2\right) \varphi^{2} d \sigma \\
& +C \int_{\widetilde{\mathbb{T}}_{x_{0}, \mathfrak{t}}} \mathfrak{t}^{2} \widetilde{k}_{\sigma}^{4} \varphi^{2} d \sigma .
\end{aligned}
$$

We break the last term into

$$
\begin{aligned}
\int_{\widetilde{\mathbb{T}}_{x_{0}, \mathfrak{t}}} \mathfrak{t}^{2} \widetilde{k}_{\sigma}^{4} \varphi^{2} d \sigma & =\int_{\widetilde{\mathbb{T}}_{x_{0}, \mathrm{t}} \cap B_{R}} \mathfrak{t}^{2} \widetilde{k}_{\sigma}^{4} \varphi^{2} d \sigma+\int_{\widetilde{\mathbb{T}}_{x_{0}, \mathfrak{t}} \cap\left(B_{3 R} \backslash B_{R}\right)} \mathfrak{t}^{2} \widetilde{k}_{\sigma}^{4} \varphi^{2} d \sigma \\
& =\int_{\widetilde{\mathbb{T}}_{x_{0}, \mathrm{t}} \cap B_{R}} \mathfrak{t}^{2} \widetilde{k}_{\sigma}^{4} d \sigma+\int_{\widetilde{\mathbb{T}}_{x_{0}, \mathfrak{t}} \cap\left(B_{3 R} \backslash B_{R}\right)} \mathfrak{t}^{2} \widetilde{k}_{\sigma}^{4} \varphi^{2} d \sigma
\end{aligned}
$$

as $\varphi$ is zero outside the ball $B_{3 R}$. 
The second integral is bounded since, by the argument based on the interior estimates of Ecker and Huisken, discussed previously, $\widetilde{k}_{\sigma}$ is uniformly bounded for $\widetilde{\mathbb{T}}_{x_{0}, \mathrm{t}} \cap\left(B_{3 R} \backslash B_{R}\right)$.

The first integral is controlled by interpolating between a possibly large multiple of $\int \mathfrak{t}^{2} \widetilde{k}^{2} d \sigma$, which is bounded, and a small fraction of $\int \mathfrak{t}^{2} \widetilde{k}_{\sigma \sigma \sigma}^{2} d \sigma$ $\widetilde{\mathbb{T}}_{x_{0}, \mathrm{t}} \cap B_{R}$

which is less than $\int_{\widetilde{\mathbb{T}}_{x_{0}, \mathrm{t}}} \mathfrak{t}^{2} \widetilde{k}_{\sigma \sigma \sigma}^{2} \varphi^{2} d \sigma$.

Hence, we conclude (in the case $\mathbb{T}_{\infty}$ is a halfline the 3-point contribution is not present)

$$
\begin{aligned}
\frac{d}{d \mathfrak{t}} \int\left(\widetilde{\mathbb{T}}_{x_{0}, \mathrm{t}}\right. \\
\end{aligned}
$$

and dealing with the 3-point terms as in Section 3 we finally get

$$
\int_{\widetilde{\mathbb{T}}_{x_{0}, \mathrm{t}}}\left(\widetilde{k}^{2}+\widetilde{\mathrm{t}}_{\sigma}^{2}+\mathfrak{t}^{2} \widetilde{k}_{\sigma \sigma}^{2} / 2\right) \varphi^{2} d \sigma \leq C
$$

for every $\mathfrak{t} \in[0, \delta]$, with uniform constants $\delta$ and $C$. Reparametrizing the flow in time, in such a way that $\mathfrak{t}_{j_{l}}$ converges to $\delta$, we have

$$
\int_{\widetilde{\mathbb{T}}_{x_{0}, \mathrm{t}_{l}} \cap B_{R}} \widetilde{k}_{\sigma}^{2} d \sigma \leq C / \delta
$$

for every $l \in \mathbb{N}$.

Then, since $\left\|\widetilde{k}_{\sigma}\right\|_{L^{2}\left(B_{R}\right)}$ and $\widetilde{k}$ are uniformly bounded, we can finally extract a subsequence of triods converging in the $C_{\text {loc }}^{2}$ topology to $\mathbb{T}_{\infty}$.

By means of this proposition we can exclude the first case of Lemma 6.1.

Proposition 6.18. Type I singularities such that for an index $i \in\{1,2,3\}$ there exists a sequence of times $t_{j} \rightarrow T$ satisfying

$$
\left[k^{i}\left(O, t_{j}\right)\right]^{2} \geq \frac{C}{T-t_{j}}
$$

for some positive constant $C$, are not present. 
Proof. We have seen in Lemma 6.9 that $\lim _{t \rightarrow T} F(O, t)=\widehat{O}$.

Considering the triods $\widetilde{\mathbb{T}} \widehat{O}_{, t_{j}}$, where $\mathfrak{t}_{j}=\mathfrak{t}\left(t_{j}\right)$, by Proposition 6.16 we can extract a subsequence (not relabelled) $C^{2}$ converging in the ball of radius $\sqrt{2 C}$ to a limit set with zero curvature, moreover this limit is not empty because the 3-point $\widetilde{F}_{\widehat{O}}\left(O, \mathfrak{t}_{j}\right)$ belongs to such ball for every $j \in \mathbb{N}$, again by Lemma 6.9.

We have now a contradiction because at these points

$$
\left[\widetilde{k}^{i}\left(O, \mathfrak{t}_{j}\right)\right]^{2}=2\left(T-t_{j}\right)\left[k^{i}\left(O, t_{j}\right)\right]^{2} \geq 2\left(T-t_{j}\right) \frac{C}{T-t_{j}}=2 C>0,
$$

hence, as the convergence is in the $C^{2}$ topology, the curvature of the limit cannot be zero.

Following Ilmanen, we deal now with the second case of Lemma 6.1.

Proposition 6.18. There are no Type I singularities such that

$$
\max _{\mathbb{T}_{t}} k^{2}=\frac{\varepsilon(t)}{T-t}
$$

and $\varepsilon:[0, T) \rightarrow \mathbb{R}$ goes to zero as $t \rightarrow T$.

Proof. By means of Lemma 6.2 we know that there exists an index $i \in$ $\{1,2,3\}$ and a sequence of times $t_{j} \nearrow T$ such that

$$
k^{2}(p, t) \leq\left[k^{i}\left(O, t_{j}\right)\right]^{2} \text { for every } t \leq t_{j}, p \in \mathbb{T} \text { and }\left[k^{i}\left(O, t_{j}\right)\right]^{2} \nearrow+\infty .
$$

By Lemma 6.9, the limit $\lim _{t \rightarrow T} F(O, t)=\widehat{O}$ exists and repeating the computation in its proof we get

$$
|F(O, t)-\widehat{O}| \leq 2 \sqrt{C(T-t) \varepsilon(t)}
$$

for every $t \in[0, T)$.

We can suppose that $\widehat{O}=0 \in \mathbb{R}^{2}$ and we consider the sequence of positive values $\alpha_{j}=\max _{\mathbb{T}_{t}}|k| \nearrow+\infty$, then we rescale the triods $\mathbb{T}_{t}$ as follows, let

$$
\mathbb{T}_{\mathfrak{t}}^{j}=\alpha_{j}\left(\mathbb{T}_{t_{j}+\mathfrak{t} / \alpha_{j}^{2}}\right) \quad \text { for } \mathfrak{t} \in\left[-t_{j} \alpha_{j}^{2},\left(T-t_{j}\right) \alpha_{j}^{2}\right)
$$

We see that $-t_{j} \alpha_{j}^{2} \rightarrow-\infty$ and, possibly passing to a subsequence, we can assume that $\left(T-t_{j}\right) \alpha_{j}^{2} \searrow 0$. Then, notice that, for every $j \in \mathbb{N}$ large enough, the rescaled triods $\mathbb{T}_{\mathfrak{t}}^{j}$ still move by curvature with end points $\alpha_{j} P^{i}$ for $\mathfrak{t} \in[-1,0]$. Moreover if $O^{j}(\mathfrak{t})$ is the 3-point of $\mathbb{T}_{\mathfrak{t}}^{j}$, we have

$$
\begin{aligned}
\left|O^{j}(\mathfrak{t})\right| & =\alpha_{j}\left|F\left(O, t_{j}+\mathfrak{t} / \alpha_{j}^{2}\right)\right| \\
& \leq \alpha_{j} 2 \sqrt{C\left(T-t_{j}-\mathfrak{t} / \alpha_{j}^{2}\right) \varepsilon\left(t_{j}+\mathfrak{t} / \alpha_{j}^{2}\right)} \\
& =2 \sqrt{C\left[\left(T-t_{j}\right) \alpha_{j}^{2}-\mathfrak{t}\right] \varepsilon\left(t_{j}+\mathfrak{t} / \alpha_{j}^{2}\right)}
\end{aligned}
$$


which goes to zero when $j \rightarrow \infty$ uniformly for $\mathfrak{t} \in[-1,0]$, since $\left[\left(T-t_{j}\right) \alpha_{j}^{2}-\right.$ $\mathfrak{t}] \rightarrow-\mathfrak{t}$ and $\varepsilon\left(t_{j}+\mathfrak{t} / \alpha_{j}^{2}\right) \rightarrow 0$.

We set $\varepsilon_{j}=\varepsilon\left(t_{j}\right) \geq 0$ and we denote with

$$
\rho_{j}(x, \mathfrak{t})=\frac{e^{-\frac{|x|^{2}}{4\left(\varepsilon_{j}-\mathfrak{t}\right)}}}{\sqrt{4 \pi\left(\varepsilon_{j}-\mathfrak{t}\right)}},
$$

the Huisken's backward heat kernel relative to $\left(0, \varepsilon_{j}\right) \in \mathbb{R}^{2} \times \mathbb{R}$.

Computing as in [28, Lemma 8] and [29, Section 3] we show that a subsequence of the flows $\mathbb{T}_{\mathfrak{t}}^{j}$ converges to a curvature flow $\mathbb{T}_{\mathfrak{t}}^{\infty}$ on $[-1,0]$ which is homothetic.

$$
\begin{aligned}
& \int_{-1}^{0} \int_{\mathbb{T}_{\mathfrak{t}}^{j}}\left|\underline{k}+\frac{x^{\perp}}{2\left(\varepsilon_{j}-\mathfrak{t}\right)}\right|^{2} \rho_{j}(x, \mathfrak{t}) d \sigma d \mathfrak{t} \\
& \quad=\int_{-1}^{0} \int_{\mathbb{T}_{t_{j}+\mathfrak{t} / \alpha_{j}^{2}}} \alpha_{j}\left|\frac{\underline{\underline{k}}}{\alpha_{j}}+\frac{\alpha_{j} y^{\perp}}{2\left(\varepsilon_{j}-\mathfrak{t}\right)}\right|^{2} \frac{e^{-\frac{\alpha_{j}^{2}|y|^{2}}{4\left(\varepsilon_{j}-\mathfrak{t}\right)}}}{\sqrt{4 \pi\left(\varepsilon_{j}-\mathfrak{t}\right)}} d s d \mathfrak{t} \\
& =\int_{t_{j}-1 / \alpha_{j}^{2}}^{t_{j}} \alpha_{j}^{2} \int_{\mathbb{T}_{t}} \alpha_{j}\left|\frac{\underline{k}}{\alpha_{j}}+\frac{\alpha_{j} y^{\perp}}{2(T-t) \alpha_{j}^{2}}\right|^{2} \frac{e^{-\frac{\alpha_{j}^{2}|y|^{2}}{4(T-t) \alpha_{j}^{2}}}}{\sqrt{4 \pi(T-t) \alpha_{j}^{2}}} d s d t \\
& =\int_{t_{j}-1 / \alpha_{j}^{2}}^{t_{j}} \int_{\mathbb{T}_{t}}\left|\underline{k}+\frac{y^{\perp}}{2(T-t)}\right|^{2} \rho_{0}(y, t) d s d t
\end{aligned}
$$

where $\rho_{0}$ is the backward heat kernel relative to $(0, T) \in \mathbb{R}^{2} \times \mathbb{R}$.

By the integrated monotonicity formula (6.4), this last term is equal to

$\int_{\mathbb{T}_{t_{j}-1 / \alpha_{j}^{2}}} \rho_{0}\left(y, t_{j}-1 / \alpha_{j}^{2}\right) d s-\int_{\mathbb{T}_{t_{j}}} \rho_{0}\left(y, t_{j}\right) d s+\sum_{i=1}^{3} \int_{t_{j}-1 / \alpha_{j}^{2}}^{t_{j}} \frac{\left\langle P^{i} \mid \tau^{i}(1, t)\right\rangle}{2(T-t)} \rho_{0}\left(P^{i}, t\right) d t$

and this last expression goes to zero when $j \rightarrow \infty$, by Lemma 6.6 and 6.5.

Thus, for almost every $\mathfrak{t} \in[-1,0]$ we have that

$$
\lim _{j \rightarrow \infty} \int_{\mathbb{T}_{\mathfrak{t}}^{j}}\left|\underline{k}+\frac{x^{\perp}}{2\left(\varepsilon_{j}-\mathfrak{t}\right)}\right|^{2} \rho_{j}(x, \mathfrak{t}) d \sigma=0 .
$$

Now, all the flows $\mathbb{T}_{\mathfrak{t}}^{j}$ have uniformly bounded curvature for $\mathfrak{t} \in[-1,0]$ since by construction,

$$
\max _{\mathbb{T}_{\mathfrak{t}}^{j}} k^{2}=\alpha_{j}^{-2} \max _{\mathbb{T}_{t_{j}+\alpha_{j}^{2}}} k^{2} \leq \alpha_{j}^{-2} \max _{\mathbb{T}_{t_{j}}} k^{2}=\alpha_{j}^{-2}\left[k^{i}\left(O, t_{j}\right)\right]^{2}=1
$$


hence, repeating the local length estimate of Lemma 6.10 and the convergence argument in the proof of Proposition 6.16 (interior estimates of Ecker and Huisken plus the special treatment of the 3-point terms, see Remark 6.15), we can extract a subsequence of the flows converging in the $C_{\text {loc }}^{2}$ topology to a curvature flow of triods $\mathbb{T}_{\mathfrak{t}}^{\infty}$ in $[-, 1,0]$ which, by limit (6.11) must satisfy

$$
\int_{\mathbb{T}_{\mathfrak{t}}^{\infty}}\left|\underline{k}-x^{\perp} / 2 \mathfrak{t}\right|^{2} \rho_{j}(x, \mathfrak{t}) d \sigma=0
$$

for almost every $\mathfrak{t} \in[-1,0]$. By Lemma 5.2, it follows that for every $\mathfrak{t} \in[-1,0]$, every triod $\mathbb{T}_{\mathfrak{t}}^{\infty}$ is composed of three halflines by the origin of $\mathbb{R}^{2}$ and it has zero curvature.

Looking in particular at $\mathbb{T}_{0}^{\infty}$ which is the limit in the $C^{2}$ topology of the triods $\alpha_{j} \mathbb{T}_{t_{j}}$, we finally have a contradiction since these latter have at least one squared curvature equal to one at their 3-points (which converge to the origin) by the initial choice of the sequence $t_{j} \nearrow T$.

The rest of the section is concerned with the last case of Lemma 6.1, so, from now on, we suppose that the maximum of the curvature is not taken at the 3-point and that $\lim _{t \rightarrow T}(T-t) k^{2}(O, t)=0$, otherwise we are in the first case. Since in this case we have no bound on the tangential velocity at a maximum point of the curvature, we modify our function $F$ to keep it under control. ODE's,

Let $\theta^{i}:[0,1] \times[0, T) \rightarrow[0,1]$ be the smooth solutions of the following

$$
\frac{\partial \theta^{i}(x, t)}{\partial t}=\frac{\lambda^{i}(0, t)(1-x)-\lambda^{i}\left(\theta^{i}(x, t), t\right)}{\left|\gamma_{x}^{i}\left(\theta^{i}(x, t), t\right)\right|}
$$

with the initial conditions $\theta^{i}(x, 0)=x$. These functions are well defined on the interval $[0, T)$ since the curves $\gamma^{i}$ and all their derivatives are smooth and $\gamma_{x}^{i} \neq 0$, moreover $\theta^{i}(0, t)=0, \theta^{i}(1, t)=1$ are barrier solutions, as $\lambda^{i}(1, t)=0$.

Defining naturally, via $\theta^{i}$, a function $\theta: \mathbb{T} \times[0, T) \rightarrow \mathbb{T}$, the function $G(p, t)=F(\theta(p, t), t)$ satisfies

$$
\begin{aligned}
\frac{\partial G(p, t)}{\partial t}=\underline{k}+\underline{\lambda}+F_{p} \frac{\partial \theta}{\partial t} & =\underline{k}+\lambda \tau+\frac{F_{p}}{\left|F_{p}\right|}(\lambda(O, t)(1-x(p))-\lambda) \\
& =\underline{k}(G(p, t))+\lambda(O, t)(1-x(p)) \tau(G(p, t))
\end{aligned}
$$

where $x(p)$ denotes the value in the interval $[0,1]$ such that for a certain index $i \in\{1,2,3\}, F(p, t)=\gamma^{i}(x(p), t)$.

Notice that, by construction, $G(O, t)=F(O, t)$ and $G\left(P^{i}, t\right)=F\left(P^{i}, t\right)$.

From now on in all the rest of this section we will refer all the quantities $\tau, v$, $k, \lambda$, etc... to the new parametrization of the flow $G$, that is, for instance $k(p, t)$ is the curvature of the triod $\mathbb{T}_{t}$ at the point $G(p, t)$ and so on.

Denoting with $\underline{w}(p, t)$ the new velocity of the point $G(p, t) \in \mathbb{T}_{t}$ and with $\underline{\eta}(p, t)=\eta(p, t) \tau(p, t)$ its tangential part, that is, $\underline{w}=\underline{k}+\underline{\eta}$, we have

$$
\underline{\eta}(p, t)=\eta(p, t) \tau(p, t)=\lambda(O, t)(1-x(p)) \tau(p, t),
$$

then clearly $\eta^{i}(O, t)=\lambda^{i}(O, t)$ and $\eta\left(P^{i}, t\right)=0$. 
By assumption (6.1) and the relations between $k$ and $\lambda$ at the 3-point, the new velocity $w(p, t)=\underline{k}(p, t)+\lambda(O, t)(1-x(p)) \tau(p, t)$ is uniformly bounded by $C / \sqrt{T-t}$.

The evolution equation for the curvature has to be modified in

$$
\partial_{t} k=k_{s s}+\eta k_{s}+k^{3}
$$

and the commutation law as follows,

$$
\partial_{t} \partial_{s}=\partial_{s} \partial_{t}+\left(k^{2}-\eta_{s}\right) \partial_{s} .
$$

Rescaling $G$, like we did before for $F$, we define the map $\widetilde{G}_{x_{0}}: \mathbb{T} \times$ $[-1 / 2 \log T,+\infty) \rightarrow \mathbb{R}^{2}$ as

$$
\widetilde{G}_{x_{0}}(p, \mathfrak{t})=\frac{G(p, t)-x_{0}}{\sqrt{2(T-t)}} \quad \mathfrak{t}(t)=-\frac{1}{2} \log (T-t) .
$$

Notice that the rescaled triods are not changed

$$
\widetilde{\mathbb{T}}_{x_{0}, \mathrm{t}}=\frac{\mathbb{T}_{t}-x_{0}}{\sqrt{2(T-t)}}
$$

but now they evolve according to the equation

$$
\frac{\partial}{\partial \mathfrak{t}} \widetilde{G}_{x_{0}}(p, \mathfrak{t})=\underline{\widetilde{w}}(p, \mathfrak{t})+\widetilde{G}_{x_{0}}(p, \mathfrak{t})
$$

where $\underline{\widetilde{w}}(p, \mathfrak{t})$ is the rescaled velocity given by

$$
\underline{\widetilde{w}}(p, \mathfrak{t})=\frac{\underline{w}(p, t(\mathfrak{t}))}{\sqrt{2(T-t(\mathfrak{t}))}}=\underline{\widetilde{k}}+\widetilde{\lambda}(O, t(\mathfrak{t}))(1-x(p)) \tau=\widetilde{k} \nu+\widetilde{\eta} \tau .
$$

The rescaled curvature evolves according to the following equation,

$$
\partial_{\mathrm{t}} \widetilde{k}=\widetilde{k}_{\sigma \sigma}+\widetilde{\eta} \widetilde{k}_{\sigma}+\widetilde{k}^{3}-\widetilde{k}
$$

and the commutation law is

$$
\partial_{\mathfrak{t}} \partial_{\sigma}=\partial_{\sigma} \partial_{\mathfrak{t}}+\left(\widetilde{k}^{2}-\widetilde{\eta}_{\sigma}-1\right) \partial_{\sigma} .
$$

We call $p_{t} \neq O$ a point in $\mathbb{T}$ where the curvature achieves its maximum, so by hypothesis

$$
\frac{1}{2(T-t)} \leq k^{2}\left(p_{t}, t\right) \leq \frac{C}{2(T-t)} .
$$

We define the map $\uparrow: \mathbb{T} \rightarrow \mathbb{R}^{2}$ as follows

$$
\widehat{p}=\lim _{t \rightarrow T} G(p, t)
$$


for every $p \in \mathbb{T}$. Such limit exists for every $p \in \mathbb{T}$ since, for every $0 \leq t_{1} \leq$ $t_{2}<T$, we have

$$
\begin{aligned}
\left|G\left(p, t_{1}\right)-G\left(p, t_{2}\right)\right| & =\left|\int_{t_{1}}^{t_{2}} \frac{\partial G}{\partial t} d t\right| \\
& \leq \int_{t_{1}}^{t_{2}}|\underline{w}(p, t)| d t \leq \int_{t_{1}}^{t_{2}} \sqrt{\frac{C}{T-t}} d t \leq 2 \sqrt{C\left(T-t_{1}\right)}
\end{aligned}
$$

and then

$$
|G(p, t)-\widehat{p}| \leq 2 \sqrt{C(T-t)}
$$

for every $t$. Notice that the definition of $\widehat{O}$ coincides with the one in Lemma 6.9, since $G(O, t)=F(O, t)$.

LeMMA 6.19.

(1) The map $\uparrow: \mathbb{T} \rightarrow \mathbb{R}^{2}$ is continuous.

(2) For every $p \in \mathbb{T}$ all the triods $\widetilde{\mathbb{T}}_{\widehat{p}, \mathrm{t}}$ intersect the closed ball $B_{\sqrt{2 C}}$ centered at the origin of $\mathbb{R}^{2}$, indeed, $\widetilde{G}_{\widehat{p}}(p, \mathfrak{t})$ belongs to such ball for every $\mathfrak{t} \in$ $[-1 / 2 \log T,+\infty)$.

(3) The point $\widehat{O}$ is different from $P^{1}, P^{2}, P^{3}$ (which coincide respectively with $\widehat{P}^{1}$, $\widehat{P}^{2}, \widehat{P}^{3}$ ).

\section{PROOF.}

(1) Since all the maps $G(\cdot, t)$ are continuous and inequality (6.13) says that they converge uniformly as $t \rightarrow T$, the map ${ }^{\wedge}: \mathbb{T} \rightarrow \mathbb{R}^{2}$ is also continuous.

(2) By inequality (6.13), we have

$$
\left|\widetilde{G}_{\hat{p}}(p, \mathfrak{t})\right|=\frac{|G(p, t(\mathfrak{t}))-\hat{p}|}{\sqrt{2(T-t(\mathfrak{t}))}} \leq \frac{2 \sqrt{C(T-t(\mathfrak{t}))}}{\sqrt{2(T-t(\mathfrak{t}))}}=\sqrt{2 C}
$$

and the second statement is proved.

(3) The point $\widehat{O}$ cannot coincides with any of the end points $P^{i}$, let us say $P^{1}$, otherwise, rescaling the triods around such end point, by Proposition 6.16 we can find a subsequence of the rescaled triods converging in the $C_{\text {loc }}^{2}$ topology to a halfline from the origin of $\mathbb{R}^{2}$, but Lemma 6.9 says that since $\widehat{O}=P^{1}$ the 3-point cannot disappear in the limit, which is a contradiction.

Following Stone [37], we define the function $\Theta(p, t)$ as

$$
\Theta\left(p, t_{j}\right)=\int_{\mathbb{T}_{t_{j}}} \rho_{\widehat{p}} d s=\frac{1}{\sqrt{2 \pi}} \int_{\widetilde{\mathbb{T}}_{\widehat{p}, t_{j}}} \tilde{\rho} d \sigma
$$

for every $p \in \mathbb{T}$ and the limiting heat density as the limit for $t \rightarrow T$ of

$$
\widehat{\Theta}(p)=\lim _{t \rightarrow T} \Theta(p, t),
$$

if it exists.

Notice that since $p \mapsto \widehat{p}$ is continuous, all the maps $p \mapsto \Theta(p, t)$ are also continuous, for every $t \in[0, T)$ 
Proposition 6.20. The limit $\widehat{\Theta}(p)$ exists and it is finite for every $p \in \mathbb{T}$, moreover, $\widehat{\Theta}(p)$ can take only the values $1 / 2,1,3 / 2$.

Proof. As in Lemma 6.6, we define the function $b: \mathbb{T} \times[0, T) \rightarrow \mathbb{R}$ as follows,

$$
b(p, t)=\int_{t}^{T} \sum_{i=1}^{3}\left\langle\frac{P^{i}-\hat{p}}{2(T-\xi)} \mid \tau^{i}(1, \xi)\right\rangle \rho_{\widehat{p}}\left(P^{i}, \xi\right) d \xi
$$

then Lemma 6.5 says that $b$ is bounded and for every $p \in \mathbb{T}$ we have $\lim _{t \rightarrow T} b(p, t)=0$. The monotonicity formula (6.3) can be rewritten as

$$
\frac{d}{d t}(\Theta(p, t)+b(p, t))=-\int_{\mathbb{T}_{t}}\left|\underline{k}+\frac{(x-\widehat{p})^{\perp}}{2(T-t)}\right|^{2} \rho_{\widehat{p}} d s \leq 0,
$$

hence, being non increasing and bounded from below, the functions $(\Theta(\cdot, t)+$ $b(\cdot, t))$ pointwise converge on all $\mathbb{T}$ when $t \rightarrow T$. Since we have seen that $b(\cdot, t)$ also pointwise converge to zero everywhere, the limit $\widehat{\Theta}(p)$ exists for every $p$.

We consider now, by means of Proposition 6.16, a sequence of times $t_{j} \nearrow T$ such that the rescaled triods $\widetilde{\mathbb{T}}_{\widehat{p}, \mathrm{t}_{j}}$ converge in the $C_{\mathrm{loc}}^{2}$ topology to some zero curvature set $\mathbb{T}_{\infty}$.

Taking into account that $\widetilde{k}$ is uniformly bounded, we compute

$$
\begin{aligned}
& \frac{d}{d \mathfrak{t}} \int_{\widetilde{\mathbb{T}_{p}, \mathrm{t}}} e^{-|x|} d \sigma=\int_{\widetilde{\mathbb{T}}_{\widehat{p}, \mathrm{t}}} e^{-|x|}\left(1-\widetilde{k}^{2}-\frac{\langle x \mid \underline{\tilde{k}}+x\rangle}{|x|}\right) d \sigma \\
& \leq \int_{\widetilde{\mathbb{T}} \widehat{p}, \mathrm{t}} e^{-|x|}(C-|x|) d \sigma \\
& \leq C \int_{\widetilde{\mathbb{T}} \widehat{p}, \mathrm{f}} \int_{B_{C+1}} e^{-|x|} d \sigma-\int_{\widetilde{\mathbb{T}}_{\widehat{p}, \mathrm{t}} \backslash B_{C+1}} e^{-|x|} d \sigma \\
& \leq(C+1) \int_{\widetilde{\mathbb{T}} \widehat{p}, \mathrm{t}} e^{-|x|} d \sigma-\int_{\mathbb{\mathbb { T }}_{\widehat{p}, \mathrm{t}}} e^{-|x|} d \sigma .
\end{aligned}
$$

This means that if

$$
\frac{d}{d \mathfrak{t}} \int_{\widetilde{\mathbb{T}}, \mathfrak{t}} e^{-|x|} d \sigma \geq 0
$$

then

$$
\int_{\widetilde{\mathbb{T}}, \mathrm{t}} e^{-|x|} d \sigma \leq(C+1) \int_{\widetilde{\mathbb{T}}_{\widehat{p}, \mathrm{t}} \cap B_{C+1}} e^{-|x|} d \sigma
$$


which clearly implies that for every $\mathfrak{t} \in[-1 / 2 \log (T-t),+\infty)$

$$
\begin{aligned}
& \int_{\widetilde{\mathbb{T}} \widehat{p}, \mathrm{t}} e^{-|x|} d \sigma \leq(C+1) \int_{\widetilde{\mathbb{T}} \widehat{p}, \mathrm{t}} \int_{B_{C+1}} e^{-|x|} d \sigma \\
& \leq(C+1) \quad \int \quad e^{\frac{C^{2}-|x|^{2}}{2}} d \sigma \\
& \widetilde{\mathbb{T}}_{\hat{p}, \mathrm{t}} \cap B_{C+1} \\
& =(C+1) e^{C^{2} / 2} \quad \int e^{-\frac{|x|^{2}}{2}} d \sigma \\
& \widetilde{\mathbb{T}} \widehat{p, t} \cap B_{C+1} \\
& =\frac{(C+1) e^{C^{2} / 2}}{\sqrt{2 \pi}} \Theta(p, t(\mathfrak{t})) \leq D
\end{aligned}
$$

where the constant $D$ does not depend on $\mathfrak{t}$, since we have seen that $\Theta(p, t)$ converges as $t \rightarrow T$.

Now, we subdivide $\widetilde{\mathbb{T}} \widehat{p}, \mathrm{t}_{j}$ into annular pieces $\widetilde{\mathbb{T}}_{\hat{p}, \mathrm{t}_{j}}^{n}$ for $n \in \mathbb{N}$, as follows: $\widetilde{\mathbb{T}}_{\widehat{p}, \mathrm{t}_{j}}^{0}=\widetilde{\mathbb{T}}_{\widehat{p}, \mathrm{t}_{j}} \cap B_{1} \quad \widetilde{\mathbb{T}}_{\widehat{p}, \mathrm{t}_{j}}^{n}=\left\{x \in \widetilde{\mathbb{T}}_{\widehat{p}, \mathrm{t}_{j}}\left|2^{n-1} \leq\right| x \mid<2^{n}\right\} \quad$ for every $n \geq 1$.

By the computations above $\int_{\widetilde{\mathbb{T}} \widehat{p}_{\mathrm{t}_{j}}} e^{-|x|} d \sigma$ is bounded independently of $j \in \mathbb{N}$, then trivially

$$
\mathcal{H}^{1}\left(\widetilde{\mathbb{T}}_{\widehat{p}, \mathfrak{t}}^{n}\right) \leq e^{\left(2^{n}\right)} \int_{\widetilde{\mathbb{T}}_{\widehat{p}, \mathfrak{t}}^{n}} e^{-|x|} d \sigma \leq A e^{\left(2^{n}\right)},
$$

hence, for every $n \geq 1$ we have

$$
\int_{\widetilde{\mathbb{T}}_{\widehat{p}, \mathrm{t}}^{n}} e^{-\frac{|x|^{2}}{2}} d \sigma \leq e^{-\frac{1}{2}\left(2^{n-1}\right)^{2}} A e^{\left(2^{n}\right)}=A e^{\left(2^{n}-2^{2 n-3}\right)} .
$$

Now, for every $\varepsilon>0$ we can find a number $n_{0} \in \mathbb{N}$ such that $\sum_{n=n_{0}}^{\infty} A e^{\left(2^{n}-2^{2 n-3}\right)} \leq$ $\varepsilon$, that is, if $R \geq 2^{n_{0}-1}$,

$$
\int_{\widetilde{\mathbb{T}} \widehat{p}, \backslash B_{R}} e^{-\frac{|x|^{2}}{2}} d \sigma \leq \varepsilon
$$

for every $\mathfrak{t} \in[-1 / 2 \log (T-t),+\infty)$. Since we have

$$
\Theta\left(p, t_{j}\right)=\int_{\mathbb{T}_{t_{j}}} \rho_{\widehat{p}} d s=\frac{1}{\sqrt{2 \pi}} \int_{\widetilde{\mathbb{T}}_{\widehat{p}, t_{j}}} \tilde{\rho} d \sigma
$$


we get

$$
\left|\Theta\left(p, t_{j}\right)-\frac{1}{\sqrt{2 \pi}} \underset{\widetilde{\mathbb{T}} \widehat{p}_{, \mathrm{t}_{j}} \cap B_{R}}{ } \tilde{\rho} d \sigma\right| \leq \frac{1}{\sqrt{2 \pi}} \underset{\widetilde{\mathbb{T}_{p}, \mathrm{t}_{j} \backslash B_{R}}}{ } \int_{\rho} \tilde{\rho} d \sigma \leq \frac{\varepsilon}{\sqrt{2 \pi}} .
$$

Since $\Theta\left(p, t_{j}\right)$ converges to $\widehat{\Theta}(p)$ and the measures $\mathcal{H}^{1}\left\llcorner\widetilde{\mathbb{T}} \widehat{p}, t_{j}\right.$ go to $\mathcal{H}^{1}\left\llcorner\mathbb{T}_{\infty}\right.$, by Proposition 6.16 , we obtain

$$
\left|\widehat{\Theta}(p)-\frac{1}{\sqrt{2 \pi}} \int_{\mathbb{T}_{\infty} \cap B_{R}} \tilde{\rho} d \sigma\right| \leq \frac{\varepsilon}{\sqrt{2 \pi}}
$$

and sending $R$ to $+\infty$,

$$
\left|\widehat{\Theta}(p)-\frac{1}{\sqrt{2 \pi}} \int_{\mathbb{T}_{\infty}} \tilde{\rho} d \sigma\right| \leq \frac{\varepsilon}{\sqrt{2 \pi}},
$$

hence, by the arbitrariness of $\varepsilon$,

$$
\widehat{\Theta}(p)=\frac{1}{\sqrt{2 \pi}} \int_{\mathbb{T}_{\infty}} \tilde{\rho} d \sigma .
$$

As $\mathbb{T}_{\infty}$ is one of the sets of Proposition 6.16 , the claim follows from the fact that $\int_{0}^{+\infty} e^{-y^{2} / 2} d y=\sqrt{\pi / 2}$.

We consider the following three parts of $\mathbb{T}$,

$$
\begin{aligned}
W^{-} & =\{p \in \mathbb{T} \mid \widehat{\Theta}(p)=1 / 2\} \\
W & =\{p \in \mathbb{T} \mid \widehat{\Theta}(p)=1\} \\
W^{+} & =\{p \in \mathbb{T} \mid \widehat{\Theta}(p)=3 / 2\} .
\end{aligned}
$$

Since by Lemma 6.19 the point $\widehat{O}$ is distinct from $P^{1}, P^{2}$ and $P^{3}$ (which respectively coincide with $\widehat{P}^{1}, \widehat{P}^{2}$ and $\widehat{P}^{3}$ ), if $\widehat{p}$ is different from all the $P^{i}$ and $\widehat{O}$, taking into account Proposition 6.16, every $C_{\text {loc }}^{2}$ converging subsequence of the rescaled triods $\widetilde{\mathbb{T}}_{\widehat{p}, \mathrm{t}}$ must go to a straight line, as the 3 -point and the end points go to infinity (recall Lemma 6.9). Hence $\widehat{\Theta}(p)=1$.

If $\widehat{p}=P^{1}$, for instance, then $\widehat{\Theta}(p)=\widehat{\Theta}\left(P^{1}\right)$ which is equal to $1 / 2$ since the 3-point of the rescaled triods goes to infinity and the end point $P^{1}$ is fixed, hence the limit is a halfline from the origin of $\mathbb{R}^{2}$. 
Finally, with the same argument (now the three end points go to infinity) if $\widehat{p}=\widehat{O}$ then $\widehat{\Theta}(p)=3 / 2$.

We get then the following equalities

$$
\begin{aligned}
W^{-} & =\left\{p \in \mathbb{T} \mid \widehat{p} \in\left\{P^{i}\right\}\right\} \\
W & =\left\{p \in \mathbb{T} \mid \widehat{p} \notin\left\{P^{i}, \widehat{O}\right\}\right\} \\
W^{+} & =\{p \in \mathbb{T} \mid \widehat{p}=\widehat{O}\}
\end{aligned}
$$

moreover, notice that they are non empty with $W^{-}, W^{+}$closed and $W$ open in $\mathbb{T}$, as the map $p \mapsto \widehat{p}$ is continuous.

Fixed $x_{0} \in \mathbb{R}^{2}$, if $\lim _{t \rightarrow T} \frac{\left|\widehat{p}_{t}-x_{0}\right|^{2}}{T-t}<+\infty$ (we recall that $p_{t} \in \mathbb{T}$ is a maximum point of $k^{2}$ at time $t \in[0, T)$ ) we can find a sequence of points $p_{j}=p_{t_{j}} \in \mathbb{T}$ such that $\left|\widehat{p}_{j}-x_{0}\right|^{2} \leq 2 D\left(T-t_{j}\right)$, for some constant $D$.

Rescaling around $x_{0}$ like in the proof of Proposition 6.17, we see that the limit triod is not empty because the point $\widetilde{G}_{x_{0}}\left(p_{j}, \mathfrak{t}_{j}\right)$ belongs to the closed ball of radius $\sqrt{2 C}+\sqrt{D}$, indeed

$$
\begin{aligned}
\left|\widetilde{G}_{x_{0}}\left(p_{j}, \mathfrak{t}_{j}\right)\right|=\frac{\left|G\left(p_{j}, t_{j}\right)-x_{0}\right|}{\sqrt{2\left(T-t_{j}\right)}} & \leq \frac{\left|G\left(p_{j}, t_{j}\right)-\widehat{p}_{j}\right|}{\sqrt{2\left(T-t_{j}\right)}}+\frac{\left|\widehat{p}_{j}-x_{0}\right|}{\sqrt{2\left(T-t_{j}\right)}} \\
& \leq \frac{2 \sqrt{C\left(T-t_{j}\right)}}{\sqrt{2\left(T-t_{j}\right)}}+\frac{\sqrt{2 D\left(T-t_{j}\right)}}{\sqrt{2\left(T-t_{j}\right)}} \\
& =\sqrt{2 C}+\sqrt{D},
\end{aligned}
$$

by Lemma 6.19. Moreover,

$$
\widetilde{k}^{2}\left(p_{j}, \mathfrak{t}_{j}\right)^{2}=2\left(T-t_{j}\right) k^{2}\left(p_{j}, t_{j}\right)^{2}=2\left(T-t_{j}\right) \max _{\mathbb{T}_{j}} k^{2} \geq 2\left(T-t_{j}\right) \frac{1}{2\left(T-t_{j}\right)}=1,
$$

by our initial assumption, hence, we can exclude this situation as we did in Proposition 6.17.

Thus, we can assume that for every $x_{0} \in \mathbb{R}^{2}$

$$
\lim _{t \rightarrow T} \frac{\left|\widehat{p}_{t}-x_{0}\right|^{2}}{T-t}=+\infty
$$

in particular for $x_{0} \in\left\{P^{i}, \widehat{O}\right\}$. This implies that the points $p_{t}$ belong definitely to the set $W$, that is, $\widehat{\Theta}\left(p_{t}\right)=1$. 
We consider then a sequence of times $t_{j} \nearrow T$, such that the sequence of points $p_{j}=p_{t_{j}} \in \mathbb{T}$ converges to some $p \in \mathbb{T}$. By construction, $\max _{\mathbb{T}_{t_{j}}} k^{2}=$ $k^{2}\left(p_{j}, t_{j}\right)$, moreover we can suppose that all the points $p_{j}$ belong to the same curve of $\mathbb{T}$ and to the set $W$, that is, $\widehat{\Theta}\left(p_{j}\right)$ is constantly equal to 1. By the uniform continuity of the map $G$, we have $G\left(p_{j}, t_{j}\right) \rightarrow \widehat{p}$ and $\widehat{p}_{j} \rightarrow \widehat{p}$.

Assumption (6.15) says also that for every radius $R>0$ there exists an index $j_{0} \in \mathbb{N}$ such that all the sets $\widetilde{\mathbb{T}}_{\widehat{p}_{j}, \mathrm{t}_{j}} \cap B_{3 R}$ do not contain neither the 3-point nor the three end points, for every $j \geq j_{0}$. Indeed,

$$
\begin{aligned}
\left|\widetilde{G}_{\widehat{p}_{j}}\left(O, \mathfrak{t}_{j}\right)\right|=\frac{\left|G\left(O, t_{j}\right)-\widehat{p}_{j}\right|}{\sqrt{2\left(T-t_{j}\right)}} & \geq \frac{\left|\widehat{p}_{j}-\widehat{O}\right|}{\sqrt{2\left(T-t_{j}\right)}}-\frac{\left|G\left(O, t_{j}\right)-\widehat{O}\right|}{\sqrt{2\left(T-t_{j}\right)}} \\
& \geq \frac{\left|\widehat{p}_{j}-\widehat{O}\right|}{\sqrt{2\left(T-t_{j}\right)}}-\frac{2 \sqrt{C\left(T-t_{j}\right)}}{\sqrt{2\left(T-t_{j}\right)}} \\
& \geq \frac{\left|\widehat{p}_{j}-\widehat{O}\right|}{\sqrt{2\left(T-t_{j}\right)}}-\sqrt{2 C}
\end{aligned}
$$

which goes to $+\infty$ as $j \rightarrow \infty$. The argument is the same for the three end points $P^{i}$.

Lemma 6.21. For every $\varepsilon>0$ there exists an open neighborhood $U_{\varepsilon}$ of $W^{-}$in $\mathbb{T}$ and a time $t_{\varepsilon}$ such that $\Theta(p, t) \leq 1+\varepsilon$ for every $p \in U_{\varepsilon}$ and $t \in\left[t_{\varepsilon}, T\right)$.

Proof. Suppose, by contradiction, that $t_{j} \nearrow T, p_{j}=p_{t_{j}} \rightarrow p \in W^{-}$ and $\Theta\left(p_{j}, t_{j}\right)>1+\varepsilon$ for every $j \in \mathbb{N}$. Then, we can also assume that $\widehat{p}=P^{1}$ hence, $d_{j}^{2}, d_{j}^{3}>C$ for a positive constant $C$ and $d_{j}^{1} \rightarrow 0$, where $d_{j}^{i}=\left|P^{i}-\widehat{p}_{j}\right|$.

Since on $W^{-}$the functions $b(\cdot, t)$ are locally constant, hence continuous, and by Lemma 6.5 they converge uniformly to zero in $W^{-}$which is compact, by Dini's Theorem, the functions $\Theta(\cdot, t)$, restricted to $W^{-}$, converge uniformly to $1 / 2$. Hence, fixing $\varepsilon>0$, we can find a time $t_{\varepsilon}$ and an open neighborhood $U_{\varepsilon}$ of $W^{-}$such that $\Theta\left(p, t_{\varepsilon}\right) \leq 1 / 2+\varepsilon / 2$ for every $p \in U_{\varepsilon}$ and $\Theta(p, t) \leq 1 / 2+\varepsilon$ for every $p \in W^{-}$and $t \geq t_{\varepsilon}$. Moreover, we can choose $t_{\varepsilon}$ such that

$$
\frac{1}{\sqrt{2 \pi}} \int_{C / \sqrt{2\left(T-t_{\varepsilon}\right)}}^{+\infty} e^{-y^{2} / 2} d y \leq \varepsilon / 4 .
$$

If now $p_{j} \in U_{\varepsilon} \backslash W^{-}$and $t_{\varepsilon} \leq t_{j}<T$ we estimate $\Theta\left(p_{j}, t_{j}\right)$ with equation (6.4) 
as follows,

$$
\begin{aligned}
\Theta\left(p_{j}, t_{j}\right) & =\Theta\left(p_{j}, t_{\varepsilon}\right)+\left(\Theta\left(p_{j}, t_{j}\right)-\Theta\left(p_{j}, t_{\varepsilon}\right)\right) \\
& \leq 1 / 2+\varepsilon / 2+\sum_{i=1}^{3}\left|\int_{t_{\varepsilon}}^{t_{j}}\left\langle\frac{P^{i}-\widehat{p}_{j}}{2(T-\xi)} \mid \tau^{i}(1, \xi)\right\rangle \widehat{p}_{j}\left(P^{i}, \xi\right) d \xi\right| \\
& \leq 1 / 2+\varepsilon / 2+\sum_{i=1}^{3} \frac{1}{\sqrt{2 \pi}} \int_{d_{j}^{i} / \sqrt{2\left(T-t_{\varepsilon}\right)}}^{+\infty} e^{-y^{2} / 2} d y \\
& \leq 1 / 2+\varepsilon / 2+\frac{1}{\sqrt{2 \pi}} \int_{0}^{+\infty} e^{-y^{2} / 2} d y+\sum_{i=2}^{3} \frac{1}{\sqrt{2 \pi}} \int_{d_{j}^{i} / \sqrt{2\left(T-t_{\varepsilon}\right)}}^{+\infty} e^{-y^{2} / 2} d y \\
& \leq 1+\varepsilon / 2+\frac{2}{\sqrt{2 \pi}} \int_{C / \sqrt{2\left(T-t_{\varepsilon}\right)}}^{+\infty} e^{-y^{2} / 2} d y \\
& \leq 1+\varepsilon .
\end{aligned}
$$

Since $p_{j} \in W^{-}$and $t_{j} \geq t_{\varepsilon}$ imply $\Theta\left(p_{j}, t_{j}\right) \leq \frac{1}{2}+\varepsilon$, we have a contradiction.

We are now ready to exclude the last case of Lemma 6.1.

Proposition 6.22. There are no Type I singularities such that the third case of Lemma 6.1holds.

Proof. After the previous discussion, we have three situations to consider.

The case $\widehat{\Theta}(p)=1$.

The points $p_{j}$ and $p$ stay in the open set $W$, as $\widehat{\Theta}(p)=1$, hence we can find a closed interval $I$ in $W$ containing $p$, so definitely the sequence $p_{j}$.

Recalling the definition of the function $b(\cdot, t)$ in formula $(6.14)$, it is easy to see that, since for every point $q \in W$ we have $\widehat{q} \notin\left\{P^{i}\right\}$, it follows that $b(\cdot, t)$ is continuous on $W$ for every $t \in[0, T)$.

As the functions $\Theta(\cdot, t)+b(\cdot, t)$ converge monotonically on the compact $I$ to the constant function 1 when $t \rightarrow T$, by the monotonicity formula (6.3), by applying Dini's Theorem such convergence is uniform. This implies that

$$
\lim _{j \rightarrow \infty} \Theta\left(p_{j}, t_{j}\right)+b\left(p_{j}, t_{j}\right)=1
$$

and coming back to the integrated monotonicity formula (6.4) we have

$$
\lim _{j \rightarrow \infty} \int_{t_{j}}^{T} \int_{\mathbb{T}_{t}}\left|\underline{k}+\frac{\left(x-\widehat{p}_{j}\right)^{\perp}}{2(T-t)}\right|^{2} \rho_{\widehat{p}_{j}} d s d t=0
$$


Passing to the rescaled version (6.8) we get

$$
\lim _{j \rightarrow \infty} \int_{\mathfrak{t}_{j}}^{+\infty} \int_{\widetilde{\mathbb{T}}_{\widehat{p}_{j}, \mathfrak{t}}}\left|\underline{\tilde{k}}+x^{\perp}\right|^{2} \widetilde{\rho} d \sigma d \mathfrak{t}=0 .
$$

Since we know that there exists an index $j_{0} \in \mathbb{N}$ such that the 3-point is outside the ball $B_{\sqrt{8 C}}$, by Lemma 6.14 we have that $\widetilde{k}_{\sigma}$ and $\widetilde{k}_{\mathfrak{t}}$ for the family $\left\{\widetilde{\mathbb{T}}_{\widehat{p}_{j}, r} \mid j>j_{0}\right.$ and $\left.r \in\left[\mathfrak{t}_{j}, \mathfrak{t}_{j}+\delta_{\sqrt{2 C}}\right]\right\}$ are uniformly bounded by a constant $D_{R}$ in the ball $B_{\sqrt{2 C}}$ (notice that this is true because the constant $D_{R}$ is independent of $x_{0}$ in Lemma 6.14). Hence, considering a value $\delta>0$ smaller than the constant $\delta_{\sqrt{2 C}}$ with $\delta D_{\sqrt{2 C}}<1 / 2$, possibly passing to a subsequence, there exist times $r_{j} \in\left[\mathfrak{t}_{j}, \mathfrak{t}_{j}+\delta\right]$ such that

$$
\lim _{j \rightarrow \infty} \int_{\widetilde{\mathbb{T}}_{\widehat{p}_{j}, r_{j}}}\left|\underline{\widetilde{k}}+x^{\perp}\right|^{2} \widetilde{\rho} d \sigma=0 .
$$

By the choice of $\delta<\delta_{\sqrt{2 C}}$ and Lemma 6.10, we can assume that the two sequences of rescaled triods $\widetilde{\mathbb{T}}_{\widehat{p}_{j}, \mathrm{t}_{j}}$ and $\widetilde{\mathbb{T}}_{\widehat{p}_{j}, r_{j}}$ both converge in the $C^{2}$ topology in the ball $B_{\sqrt{2 C}}$. Moreover, by limit (6.16) and Lemma 5.2, the sequence $\widetilde{\mathbb{T}}_{\widehat{p}_{j}, r_{j}}$ converges to a straight line passing through the origin in the ball $B_{\sqrt{2 C}}$, hence with zero curvature.

The points $\widetilde{G}_{\widehat{p}_{j}}\left(p_{j}, r_{j}\right) \in \widetilde{\mathbb{T}}_{\widehat{p}_{j}, r_{j}}$ all belong to the closed ball $B_{\sqrt{2 C}}$, according to Lemma 6.19 , then, as $\widetilde{k}^{2}\left(p_{j}, t_{j}\right) \geq 1$, we have

$$
\widetilde{k}^{2}\left(p_{j}, r_{j}\right) \geq \widetilde{k}^{2}\left(p_{j}, \mathfrak{t}_{j}\right)-\left(r_{j}-\mathfrak{t}_{j}\right)\left|\partial_{\mathrm{t}} \widetilde{k}^{2}\left(p_{j}, \xi\right)\right| \geq 1-\delta D_{\sqrt{2 C}} \geq 1 / 2
$$

for some $\xi \in\left(\mathfrak{t}_{j}, r_{j}\right)$.

Since the convergence of the rescaled triods is in the $C^{2}$ topology in $B_{\sqrt{2 C}}$, such estimate from below is clearly in contradiction with the fact that the limit triod has zero curvature, proving the thesis in this case.

The case $\widehat{\Theta}(p)=1 / 2$.

We suppose that $\widehat{p}=P^{1}$ hence, by continuity, $\widehat{p}_{j} \rightarrow P^{1}$.

By the rescaled version of the monotonicity formula we have

$$
\begin{aligned}
\frac{1}{\sqrt{2 \pi}} \int_{\mathfrak{t}_{j}}^{+\infty} \int_{\widetilde{\mathbb{T}}_{\widehat{p}_{j}, \mathrm{t}}}\left|\widetilde{k}+x^{\perp}\right|^{2} \widetilde{\rho} d \sigma d \mathfrak{t}= & \Theta\left(p_{j}, t_{j}\right)-\widehat{\Theta}\left(p_{j}\right) \\
& +\int_{t_{j}}^{T} \sum_{i=1}^{3}\left\langle\frac{P^{i}-\widehat{p}_{j}}{2(T-t)} \mid \tau^{i}(1, t)\right\rangle \rho_{\widehat{p}_{j}}\left(P^{i}, t\right) d t \\
\leq & \Theta\left(p_{j}, t_{j}\right)-1+\frac{1}{\sqrt{2 \pi}} \sum_{i=1}^{3} \int_{d_{j}^{i} / \sqrt{2\left(T-t_{j}\right)}}^{+\infty} e^{-y^{2} / 2} d y
\end{aligned}
$$

where we applied Lemma 6.5 and we substituted $\widehat{\Theta}\left(p_{j}\right)=1$. 
Taking $\varepsilon>0$, since $p_{j} \rightarrow p \in W^{-}$and $t_{j} \rightarrow T$, by Lemma 6.21 we have $\Theta\left(p_{j}, t_{j}\right) \leq 1+\varepsilon$ if $j \in \mathbb{N}$ is large enough, hence

$$
\int_{\mathfrak{t}_{j}}^{+\infty} \int_{\widetilde{\mathbb{T}}_{\widehat{p}_{j}, \mathrm{t}}}\left|\underline{\tilde{k}}+x^{\perp}\right|^{2} \widetilde{\rho} d \sigma d \mathfrak{t} \leq \varepsilon+\frac{1}{\sqrt{2 \pi}} \sum_{i=1}^{3} \int_{d_{j}^{i} / \sqrt{2\left(T-t_{j}\right)}}^{+\infty} e^{-y^{2} / 2} d y .
$$

Now, using the hypothesis that $\lim _{j \rightarrow \infty} \frac{d_{j}^{i}}{\sqrt{2\left(T-t_{j}\right)}}=+\infty$, we obtain

$$
\lim _{j \rightarrow \infty} \int_{\mathfrak{t}_{j}}^{+\infty} \int_{\widetilde{T}_{\widehat{p}_{j}, \mathfrak{t}}}\left|\underline{\widetilde{k}}+x^{\perp}\right|^{2} \widetilde{\rho} d \sigma d \mathfrak{t}=0
$$

then we conclude like in the previous case.

The case $\widehat{\Theta}(p)=3 / 2$.

We consider the evolution of only two curves of the triods $\mathbb{T}_{t}$, one of them containing all the points $p_{j}$, let us say the ones given by the curves $\gamma^{1}$ and $\gamma^{2}$. Thus, we restrict the map $G$ to the set $\mathbb{V}$ composed of these two curves of $\mathbb{T}$ and we call $\mathbb{V}_{t}$ the evolving sets in $\mathbb{R}^{2}$. The monotonicity formula in this situation reads

$$
\begin{aligned}
\frac{d}{d t} \int_{\mathbb{V}_{t}} \rho_{x_{0}}(x, t) d s= & -\int_{\mathbb{V}_{t}}\left|\underline{k}+\frac{\left(x-x_{0}\right)^{\perp}}{2(T-t)}\right|^{2} \rho_{x_{0}}(x, t) d s \\
& +\sum_{i=1}^{2}\left\langle\frac{P^{i}-x_{0}}{2(T-t)} \mid \tau^{i}(1, t)\right\rangle \rho_{x_{0}}\left(P^{i}, t\right) \\
& -\left\langle\frac{O-x_{0}}{2(T-t)} \mid \tau^{3}(0, t)\right\rangle \rho_{x_{0}}(O, t) \\
& -\lambda^{3}(0, t) \rho_{x_{0}}(O, t) .
\end{aligned}
$$

Indeed, it can be obtained by adding the contributions of the two curves $\gamma^{1}$ and $\gamma^{2}$ given by Lemma 6.3, recalling that $\gamma^{1}(0, t)=\gamma^{2}(0, t)=G(O, t)$ and $\sum_{i=1}^{3} \tau^{i}(0, t)=\sum_{i=1}^{3} \lambda^{i}(0, t)=0$.

We define the functions $\Theta_{\mathbb{V}}(\cdot, t)$ analogous to $\Theta(\cdot, t)$ but relative to the sets $\mathbb{V}_{t}$.

For every $q \in \mathbb{V}$, if $t_{j}$ is any sequence of times going to $T$, the rescaled triods $\widetilde{\mathbb{T}}_{\widehat{q}, \mathrm{t}_{j}}$ locally converge in $C_{\text {loc }}^{2}$ (up to a subsequence) to a subset of the limit of the triods $\widetilde{\mathbb{T}} \widehat{q}_{, t_{j}}$ which can be composed of a straight line or one or two halflines from the origin of $\mathbb{R}^{2}$. Hence, it follows that $\widehat{\Theta}_{\mathbb{V}}(q)=$ $\lim _{t \rightarrow T} \Theta_{\mathbb{V}}\left(q, t_{j}\right)=\widehat{\Theta}(q)$ if $q \notin W^{+}$and $\widehat{\Theta}_{\mathbb{V}}(q)=1$ if $\widehat{q}=\widehat{O}$. 
Rescaling everything as before, equation (6.17) becomes

$$
\begin{aligned}
\frac{1}{\sqrt{2 \pi}} \int_{\mathfrak{t}}^{t^{*}} \int_{\widetilde{\mathbb{V}} \widehat{p}, \xi}\left|\underline{\underline{k}}+x^{\perp}\right|^{2} \widetilde{\rho} d \sigma d \xi= & \Theta_{\mathbb{V}}(p, t)-\Theta_{\mathbb{V}}\left(p, t^{*}\right) \\
& +\int_{t}^{t^{*}} \sum_{i=1}^{2}\left\langle\frac{P^{i}-\widehat{p}}{2(T-\xi)} \mid \tau^{i}(1, \xi)\right\rangle \rho_{\widehat{p}}\left(P^{i}, \xi\right) d \xi \\
& -\int_{t}^{t^{*}}\left\langle\frac{O-\widehat{p}}{2(T-\xi)} \mid \tau^{3}(0, \xi)\right\rangle \rho_{\widehat{p}}(O, \xi) d \xi \\
& -\int_{t}^{t^{*}} \lambda^{i}(0, \xi) \rho_{\widehat{p}}(O, \xi) d \xi .
\end{aligned}
$$

Since when $t^{*} \rightarrow T, \Theta_{\mathbb{V}}\left(p, t^{*}\right) \rightarrow \widehat{\Theta}_{\mathbb{V}}(p)$ and the integral

$$
\frac{1}{\sqrt{2 \pi}} \int_{\mathfrak{t}}^{t^{*}} \int_{\widetilde{\mathbb{V}} \widehat{p}, \xi}\left|\underline{\underline{k}}+x^{\perp}\right|^{2} \widetilde{\rho} d \sigma d \xi
$$

is non decreasing and bounded above by the analogous integral for the complete triod, the function

$$
\begin{aligned}
b_{\mathbb{V}}(p, t)= & \int_{t}^{T} \sum_{i=1}^{2}\left\langle\frac{P^{i}-\widehat{p}}{2(T-\xi)} \mid \tau^{i}(1, \xi)\right\rangle \rho_{\widehat{p}}\left(P^{i}, \xi\right) d \xi \\
& -\int_{t}^{T}\left[\left\langle\frac{O-\widehat{p}}{2(T-\xi)} \mid \tau^{3}(0, \xi)\right\rangle+\lambda^{i}(0, \xi)\right] \rho_{\widehat{p}}(O, \xi) d \xi
\end{aligned}
$$

is well defined, moreover $\lim _{t \rightarrow T} b_{\mathbb{V}}(p, t)=0$.

Restricted to a closed interval around $O \in \mathbb{V}$ contained in the set $\mathbb{V} \cap(W \cup$ $\left.W^{+}\right)$, all the functions $b_{\mathbb{V}}(\cdot, t)$ are continuous, at least for $t$ close enough to $T$, otherwise we could find a sequence of times $t_{l} \nearrow T$ and points $q_{l} \rightarrow q \in$ $\mathbb{V} \cap\left(W \cup W^{+}\right)$such that

$$
\frac{\left|G\left(O, t_{l}\right)-\widehat{q}_{l}\right|}{2\left(T-t_{l}\right)} \rightarrow 0
$$

which would imply

$$
\frac{\left|\widehat{O}-\widehat{q}_{l}\right|}{2\left(T-t_{l}\right)} \leq \frac{\left|\widehat{O}-G\left(O, t_{l}\right)\right|}{2\left(T-t_{l}\right)}+\frac{\left|G\left(O, t_{l}\right)-\widehat{q}_{l}\right|}{2\left(T-t_{l}\right)} \rightarrow \sqrt{2 C}
$$

as $l \rightarrow \infty$, clearly in contradiction with assumption $(6.15)$.

Reasoning as before, the continuous functions $\Theta_{\mathbb{V}}(\cdot, t)+\left.b_{\mathbb{V}}(\cdot, t)\right|_{\mathbb{V} \cap\left(W \cup W^{+}\right)}$ converge monotonically to the function $\widehat{\Theta}_{\mathbb{V}}$, which is constantly equal to 1 on a 
compact interval around $p \in \mathbb{V}$. Hence, by Dini's Theorem, such convergence is uniform and

$$
\begin{aligned}
\frac{1}{\sqrt{2 \pi}} \int_{\mathfrak{t}_{j}}^{\infty} \int_{\widetilde{\mathbb{V}}_{\widehat{p}_{j}, \mathfrak{t}}}\left|\underline{\tilde{k}}+x^{\perp}\right|^{2} \widetilde{\rho} d \sigma d \mathfrak{t} & =\Theta_{\mathbb{V}}\left(p_{j}, t_{j}\right)+b_{\mathbb{V}}\left(p_{j}, t_{j}\right)-\widehat{\Theta}_{\mathbb{V}}\left(p_{j}\right) \\
& =\Theta_{\mathbb{V}}\left(p_{j}, t_{j}\right)+b_{\mathbb{V}}\left(p_{j}, t_{j}\right)-1
\end{aligned}
$$

implies

$$
\lim _{j \rightarrow \infty} \int_{\mathfrak{t}_{j}}^{+\infty} \int_{\widetilde{\mathbb{V}}_{\widehat{p}_{j}, \mathfrak{t}}}\left|\underline{\widetilde{k}}+x^{\perp}\right|^{2} \widetilde{\rho} d \sigma d \mathfrak{t}=0 .
$$

Then we conclude again with the same argument of the previous cases, looking at the curvature of the limit set in the ball $B_{\sqrt{2 C}}$.

Collecting together Propositions 6.17, 6.18, 6.22 we conclude this section with the following theorem.

THEOREM 6.23. Type I singularities cannot develop during the smooth flow $\mathbb{T}_{t}$ of an embedded triod in a bounded and strictly convex domain $\Omega \subset \mathbb{R}^{2}$ if the lengths of the three curves are uniformly bounded away from zero.

REMARK 6.24. All this section, if the results on the function $E$ discussed in Section 4 (in particular Theorem 4.6) hold for some network of curves with many 3-points, can be extended. The reason is that if we assume a uniform bound from below on the lengths of all the curves of such a network, in every blow up argument all but at most one of the 3-points go to infinity and vanish in the limit (the same for the family of end points).

The situation changes dramatically without the control on the lengths, for instance in Proposition 6.16 one should consider as possible limits also all the unbounded networks composed of straight segments and curves of Abresch and Langer (see Lemma 5.2) forming 120 degrees at the 3-points.

\section{7. - Type II singularities}

We suppose to be in the Type II singularity case, that is,

$$
\varlimsup_{t \rightarrow T}(T-t) \max _{\mathbb{T}_{t}} k^{2}=+\infty .
$$

We employ Hamilton's trick to get an eternal solution to the mean curvature flow.

Let us choose a sequence of times $t_{n} \in[0, T-1 / n]$ and points $p_{n} \in \mathbb{T}$ such that

$$
k^{2}\left(p_{n}, t_{n}\right)\left(T-1 / n-t_{n}\right)=\max _{\substack{t \in[0, T-1 / n] \\ p \in \mathbb{T}}} k^{2}(p, t)(T-1 / n-t) .
$$


As the curvature blows up as $t \rightarrow T$, we can choose a (not relabelled) non decreasing subsequence $t_{n}$ converging to $T$, moreover, the curvature squared $k^{2}\left(p_{n}, t_{n}\right)$ goes monotonically to $+\infty$ and we have

$$
k^{2}\left(p_{n}, t_{n}\right) t_{n} \rightarrow+\infty, \quad k^{2}\left(p_{n}, t_{n}\right)\left(T-1 / n-t_{n}\right) \rightarrow+\infty .
$$

The first limit is obvious, about the second, by assumption (7.1), for every $M>$ 0 there exists $\bar{t}<T$ and $\bar{p} \in \mathbb{T}$ such that $k^{2}(\bar{p}, \bar{t})(T-\bar{t})>2 M$, then choosing $n \in \mathbb{N}$ large enough such that $1 / n<T-\bar{t}$ and $k^{2}(\bar{p}, \bar{t})(T-\bar{t}-1 / n)>M$ we get

$$
k^{2}\left(p_{n}, t_{n}\right)\left(T-1 / n-t_{n}\right) \geq k^{2}(\bar{p}, \bar{t})(T-\bar{t}-1 / n)>M .
$$

Since $k^{2}\left(p_{n}, t_{n}\right)\left(T-1 / n-t_{n}\right)$ is an increasing sequence and $M$ was arbitrary,

$$
\lim _{n \rightarrow \infty} k^{2}\left(p_{n}, t_{n}\right)\left(T-1 / n-t_{n}\right)=+\infty .
$$

We rescale now the triods as follows: let $F_{n}: \mathbb{T} \times\left[-k^{2}\left(p_{n}, t_{n}\right) t_{n}, k^{2}\left(p_{n}, t_{n}\right)(T-\right.$ $\left.\left.1 / n-t_{n}\right)\right] \rightarrow \mathbb{R}^{2}$ be the evolution given by

$$
F_{n}(p, \mathfrak{t})=k\left(p_{n}, t_{n}\right)\left[F\left(p, \mathfrak{t} / k^{2}\left(p_{n}, t_{n}\right)+t_{n}\right)-F\left(p_{n}, t_{n}\right)\right]
$$

and $\mathbb{T}_{\mathfrak{t}}^{n}=F_{n}(\mathbb{T}, \mathfrak{t})$.

- $F_{n}\left(p_{n}, 0\right)=0, k_{n}\left(p_{n}, 0\right)=1$;

- for every $\varepsilon>0$ and $\omega>0$ there exists $\bar{n}$ such that $\max _{\mathbb{T}_{t}^{n}} k^{2} \leq 1+\varepsilon$ for every $n \geq \bar{n}$ and $\mathfrak{t} \in\left[-k^{2}\left(p_{n}, t_{n}\right) t_{n}, \omega\right]$.

Indeed (the first point is immediate), by the choice of the pair $\left(p_{n}, t_{n}\right)$ we get

$$
\begin{aligned}
k_{n}^{2}(p, t) & =\left[k\left(p_{n}, t_{n}\right)\right]^{-2} k^{2}\left(p, \mathfrak{t} / k^{2}\left(p_{n}, t_{n}\right)+t_{n}\right) \\
& \leq\left[k\left(p_{n}, t_{n}\right)\right]^{-2} k^{2}\left(p_{n}, t_{n}\right) \frac{T-1 / n-t_{n}}{T-1 / n-t_{n}-\mathfrak{t} / k^{2}\left(p_{n}, t_{n}\right)} \\
& =\frac{k^{2}\left(p_{n}, t_{n}\right)\left(T-1 / n-t_{n}\right)}{k^{2}\left(p_{n}, t_{n}\right)\left(T-1 / n-t_{n}\right)-\mathfrak{t}},
\end{aligned}
$$

if $\mathfrak{t} \in\left[-k^{2}\left(p_{n}, t_{n}\right) t_{n}, k^{2}\left(p_{n}, t_{n}\right)\left(T-1 / n-t_{n}\right)\right]$.

The claim follows as $k^{2}\left(p_{n}, t_{n}\right)\left(T-1 / n-t_{n}\right) \rightarrow+\infty$.

Proposition 7.1. The family of flows $F_{n}$ converges in the $C_{\mathrm{loc}}^{2}$ topology to an evolution by curvature of unbounded triods $\mathbb{T}_{\mathfrak{t}}^{\infty}$ (or curves, or curves with a single end point) without self-intersections, in the time interval $(-\infty,+\infty)$. Such a flow is called eternal.

Moreover, the modulus of the curvature is uniformly bounded in space and time and it takes its absolute maximum, which is 1 , at time $\mathfrak{t}=0$ at the origin of $\mathbb{R}^{2}$. 
Proof. By the previous discussion, on every bounded interval of time, the evolutions $F_{n}$ have uniformly bounded curvature, hence, reasoning like in the previous section (interior estimates of Ecker and Huisken and treatment of the 3-point terms as in the proof of Proposition 6.16), we have a uniform local bound also on $k_{\sigma}$ for the family of rescaled flows.

Passing to the limit in the $C_{\text {loc }}^{2}$ topology we then get the flow $\mathbb{T}_{\mathfrak{t}}^{\infty}$ which clearly satisfies the claimed properties on the curvature, again by the computation above.

Finally, the limit flow is embedded by Lemma 6.12 .

REMARK 7.2. It can be shown that such a limit flow is actually smooth (see Definition 5.1), Indeed, we know by standard estimates that it is smooth far from the 3-point and, being the curves $C^{2, \alpha}$ by the uniform local estimate of $k_{\sigma}$, we can apply (locally around the 3-point) a version of the small time existence and uniqueness Theorem 3.1, substituting the boundary conditions regarding the fixed end points with the smooth motion of three chosen points of the triods $\mathbb{T}_{\mathfrak{t}}^{\infty}$.

In the case of the evolution of a closed curve in the plane, it is possible to show that the limit flow arising from the analogous of this proposition is a translating unbounded curve. This conclusion is reached in two steps: first, one shows that at every time in $(-\infty,+\infty)$ the limit is a convex curve, then, by means of the Harnack estimate proved by Hamilton in [22], it follows that it is a translating flow by curvature.

In our situation convexity means that the curvature is never zero. Unfortunately, the presence of the 3-point makes troublesome the extension of these results to the evolution of triods and we can only state the following two conjectures.

Conjecture 7.3. If the curvature is zero at some point of the triod (or curve) $\mathbb{T}_{\mathfrak{t}}^{\infty}$, then it is zero along all the curve containing such a point (everywhere).

Conjecture 7.4. The triods (or curves) $\mathbb{T}_{\mathfrak{t}}^{\infty}$ move by translation.

If this last conjecture is true, we can exclude Type II singularities, indeed, it would follows that $\mathbb{T}_{\mathfrak{t}}^{\infty}$ is one of the sets of Lemmas 5.8, 5.9 or 5.10, hence, considering a pair of points $p, q$ on two curves of the triods with opposite convexity such that the segment $[p, q]$ is orthogonal to the velocity vector $w \in \mathbb{R}^{2}$ and sending them both to infinity, we can see that $E\left(\mathbb{T}_{\mathfrak{t}}^{\infty}\right)=0$, which is in contradiction with Lemma 6.12 (where the function $E$ is defined). Indeed, the distance $[p, q]$ is bounded by a constant (the three curves are pieces of the same grim reaper) and the area $A_{p, q}$ diverges.

The same argument works if the limit $\mathbb{T}_{\mathfrak{t}}^{\infty}$ is a single curve, noticing that in this case such a curve cannot have an end point, because otherwise the curvature would be zero there (such point comes from an end point of $\mathbb{T}_{t}$ ), hence the equation $k=\langle w \mid v\rangle$ would imply that the curve is a static halfline 
parallel to $w$, so with null curvature, contradicting Proposition 7.1 which says that the curvature cannot be identically zero.

REMARK 7.5. The same argument, but with a different geometric quantity, is used by Huisken in [26] to exclude Type II singularities during the motion of a single curve.

PRoposition 7.6. If Conjecture 7.4 is true then Type II singularities cannot develop during the smooth flow $\mathbb{T}_{t}$ of an embedded triod in a bounded and strictly convex domain $\Omega \subset \mathbb{R}^{2}$.

Hamilton's proof that a convex blow up of a Type II singularity (in the standard smooth case) is translating, is heavily based on the maximum principle which, as we said, is difficult to apply in the case of triods. So, it could happen that only Conjecture 7.3 can be proved and one could possibly exclude Type II singularities without actually show that the limit flow $\mathbb{T}_{t}^{\infty}$ is translating. For instance, if the curvature is always or never zero on each curve of a smooth triod, then its three curves have asymptotic tangents (indeed, they are all convex but some of them in the opposite way, by the fact that the sum of the curvatures at the 3-point is zero), hence in order to apply the previous argument based on the function $E$, it would be enough to show that two of these limit tangents, belonging to curves with opposite convexity, coincide.

Conjecture 7.7. The flow $\mathbb{T}_{t}$ of an embedded triod in a bounded and strictly convex domain $\Omega \subset \mathbb{R}^{2}$, such that the lengths of the three curves are uniformly bounded away from zero, does not develop singularities at all.

\section{8. - Open problems}

The main problem left open in the paper is Conjecture 7.4, whose validity would imply Conjectures 7.7 and 2.10, indeed, if the flow is defined for every positive time, we can show the convergence of the triods to the minimal connection between the three end points $P^{i}$.

As the total length of the triods decreases, we have the estimate

$$
\int_{0}^{+\infty} \int_{\mathbb{T}_{t}} k^{2} d s d t \leq \sum_{i=1}^{3} L^{i}(0)<+\infty,
$$

then, supposing that for a sequence of times $t_{j} \nearrow+\infty$ we have $\int_{\mathbb{T}_{j}} k^{2} d s \geq \delta$, for some $\delta>0$, and the lengths are uniformly bounded away from zero, by inequality (3.10) we get that $\int_{\mathbb{T}_{t}} k^{2} d s \geq \delta / 2$ for every $t$ belonging to a uniform time interval around every $t_{j}$. This is clearly in contradiction with the previous estimate. 
Hence, as $\lim _{t \rightarrow+\infty} \int_{\mathbb{T}_{t}} k^{2} d s=0$, by Proposition 3.17, all the derivatives of the curvature are uniformly bounded. By the usual convergence arguments, this implies that (possibly after reparametrization) the triods converge in the $C^{\infty}$ topology to a limit triod with zero curvature, which is clearly the minimal connection between the three end points (Steiner configuration).

Other questions in the paper that we would like to set are concerned with the extension of the results to all the networks, possibly with loops, with only 3-points (no 4-points or higher order points), in particular, proving an analogous of Theorem 4.6. This would also make superfluous the requirement that the ambient set $\Omega \subset \mathbb{R}^{2}$ is strictly convex.

Finally, we conclude by listing some, naturally arising, research directions.

(1) The problem of the representation/uniqueness of Brakke flows (smooth/with equality) of triods (or networks), discussed in Remark 3.23.

(2) The problem of the existence/uniqueness of a flow for an initial triod not satisfying the 120 degrees condition at the 3-point (see the introduction and Remark 3.23).

(3) The study of the singularities such that the curvature blows up but the lengths are not bounded away from zero. Such analysis requires new estimates and the classification of homothetic and translating networks moving by curvature.

(4) The "definitory" problem of the motion of the multi-points and the analysis of the collapsing situations with change of topology (see the introduction and the papers by De Giorgi [15] and Caraballo [12]).

\section{REFERENCES}

[1] U. ABRESCH - J. LANGER, The normalized curve shortening flow and homothetic solutions, J. Differential Geom. 23 (1986), 175-196.

[2] R. Adams, "Sobolev Spaces", Academic Press, New York, 1975.

[3] F. J. Almgren -J. E. TAYLOR - L. WANG, Curvature driven flows: a variational approach, SIAM J. Control Optim. 31 (1993), 387-438.

[4] S. Altschuler - M. Grayson, Shortening space curves and flow through singularities, J. Differential Geom. 35 (1992), 283-298.

[5] S. J. Altschuler, Singularities of the curve shrinking flow for space curves, J. Differential Geom. 34 (1991), 491-514.

[6] S. Angenent, Parabolic equations for curves on surfaces: curves with p-integrable curvature, Ann. of Math. 132 (1990), 451-483.

[7] S. Angenent, Parabolic equations for curves on surfaces: intersections, blow up and generalized solutions, Ann. of Math. 133 (1991), 171-215.

[8] T. Aubin, "Some Nonlinear Problems in Riemannian Geometry", Springer, 1998. 
[9] J. M. Ball - D. Kinderlehrer - P. Podio-Guidugli - M. Slemrod (eds), "Fundamental Contributions to the Continuum Theory of Evolving Phase Interfaces in Solids", Springer, Berlin, 1999.

[10] K. A. Brakke, "The Motion of a Surface by its Mean Curvature", Princeton University Press, Princeton, N.J., 1978.

[11] L. BRonsard And F. Reitich, On three-phase boundary motion and the singular limit of a vector-valued Ginzburg-Landau equation, Arch. Rat. Mech. Anal. 124 (1993), 355-379.

[12] D. G. Caraballo, "A Variational Scheme for the Evolution of Polycrystals by Curvature", Princeton University, PhD Thesis, 1996.

[13] K.-S. Chоu - X.-P. Zhu, Shortening complete plane curves, J. Differential Geom. 50 (1998), 471-504.

[14] E. De GioRgi, "Barriers, boundaries and motion of manifolds", Scuola Normale Superiore di Pisa, 1995.

[15] E. De Giorgi, Motions of partitions, In: Variational methods for discontinuous structures (Como, 1994), Progr. Nonlinear Differential Equations Appl., Vol.25, Birkhäuser, Basel, 1996, pp. 1-5.

[16] K. ECKER, Lectures on regularity for mean curvature flow, preprint, 2001.

[17] K. EcKer - G. Huisken, Mean curvature flow of entire graphs, Ann. of Math. 130 (1989), 453-471.

[18] L. C. Evans - J. Spruck, Motion of level sets by mean curvature I, J. Differential Geom. 33 (1991), 635-681.

[19] M. A. GRayson, The heat equation shrinks embedded plane curves to round points, J. Differential Geom. 26 (1987), 285-314.

[20] M. E. Gurtin, "Thermomechanics of Evolving Phase Boundaries in the Plane", Oxford Science Publication, New York, 1993.

[21] R. S. Hamilton, Four-Manifolds with positive Ricci curvature, J. Differential Geom. 24 (1986), 153-179.

[22] R. S. Hamilton, Harnack estimate for the mean curvature flow, J. Differential Geom. 41 (1995), 215-226.

[23] R. S. Hamilton, "Isoperimetric estimates for the curve shrinking flow in the plane", Modern methods in complex analysis (Princeton, NJ, 1992), Princeton University Press, Princeton, NJ, 1995, 201-222.

[24] G. HuISKen, Flow by mean curvature of convex surfaces into spheres, J. Differential Geom. 20 (1984), 237-266.

[25] G. HuISKEn, Asymptotic behavior for singularities of the mean curvature flow, J. Differential Geom. 31 (1990), 285-299.

[26] G. HuISKEn, A distance comparison principle for evolving curves, Asian J. Math. 2 (1998), 127-133.

[27] T. Ilmanen, "Elliptic regularization and partial regularity for motion by mean curvature", Mem. Amer. Math. Soc., Vol. 108(520), AMS,1994.

[28] T. Ilmanen, Singularities of Mean Curvature Flow of Surfaces, http://www.math.ethz.ch/ ilmanen/papers/sing.ps, 1995.

[29] T. Ilmanen, Lectures on Mean Curvature Flow and Related Equations, http://www.math.ethz.ch/ ilmanen/papers/notes.ps, 1998. 
[30] D. Kinderlehrer - C. Liu, Evolution of grain boundaries, Math. Models Methods Appl. Sci. 11 (2001), 713-729.

[31] J. LANGER, A Compactness Theorem for Surfaces with $L_{p}$-Bounded Second Fundamental Form, Math. Ann. 270 (1985), 223-234.

[32] A. LUNARDI, "Analytic semigroups and optimal regularity in parabolic problems, Birkhäuser Verlag, Basel, 1995.

[33] A. LUNARDi - E. SinESTRARI - W. vON WAHL, A semigroup approach to the time dependent parabolic initial-boundary value problem, Differential Integral Equations 5 (1992), 12751306.

[34] L. Simon, "Lectures on Geometric Measure Theory", Australian National University, Proc. Center Math. Anal., Vol. 3, Canberra, 1983.

[35] V. A. Solonnikov, "Boundary value problems of mathematical physics". VIII, American Mathematical Society, Providence, R.I., 1975.

[36] H. M. SONER, Motion of a set by the curvature of its boundary, J. Differential Equations 101 (1993), 313-372.

[37] A. Stone, A density function and the structure of singularities of the mean curvature flow, Calc. Var. Partial Differential Equations 2 (1994), 443-480.

[38] A. STone, A boundary regularity theorem for mean curvature flow, J. Differential Geom. 44 (1996), 371-434.

Scuola Normale Superiore

56126 Pisa, Italy

mantegazza@sns.it

Dipartimento di Matematica Università degli Studi 56127 Pisa, Italy novaga@dm.unipi.it tortorel@dm.unipi.it 